\title{
HIGH-ACCURACY \\ FINITE-DIFFERENCE SCHEMES \\ FOR LINEAR WAVE PROPAGATION
}

by

Henry Martin Jurgens

A thesis submitted in conformity with the requirements

for the degree of Doctor of Philosophy

Graduate Department of Aerospace Science and Engineering University of Toronto

(1) Copyright by Henry Martin Jurgens, 1997 
National Library

of Canada

Acquisitions and

Bibliographic Services

395 Wellington Street

Ottawa ON KIA ONA

Canada
Bibliothèque nationale du Canada

Acquisitions et services bibliographiques

395, rue Wellington Ottawa ON K1A ON4

Canada
The author has granted a nonexclusive licence allowing the National Library of Canada to reproduce, loan, distribute or sell copies of this thesis in microform, paper or electronic formats.
L'auteur a accordé une licence non exclusive permettant à la Bibliothèque nationale du Canada de reproduire, prêter, distribuer ou vendre des copies de cette thèse sous la forme de microfiche/film, de reproduction sur papier ou sur format électronique.

L'auteur conserve la propriété du droit d'auteur qui protège cette thèse. $\mathrm{Ni}$ la thèse ni des extraits substantiels de celle-ci ne doivent être imprimés ou autrement reproduits sans son autorisation. 


\title{
HIGH-ACCURACY FINITE-DIFFERENCE SCHEMES
}

\section{FOR LINEAR WAVE PROPAGATION}

Doctor of Philosophy, 1997

\author{
Henry Martin Jurgens \\ Graduate Department of Aerospace Science and Engineering \\ University of Toronto
}

\begin{abstract}
The numerical simulation of linear wave propagation and scattering can not be handled effectively using the second-order methods that are in widespread use today. To obtain accurate results these methods require grids with a resolution in the hundreds of points per wavelength to propagate a wave a distance greater than thirty wavelengths. Methods which can obtain accurate solutions without such high grid resolutions are needed for simulating long-range wave propagation.

A procedure is presented for developing high-accuracy finite-difference schemes which are optimized in order to minimize the numerical phase and amplitude errors over a given range of frequencies. These highaccuracy schemes are used to solve the two-dimensional time-domain Maxwell equations for electromagnetic wave propagation and scattering. Boundary conditions are presented which preserve the accuracy of these schemes when modeling interfaces between different materials. A stable and accurate far-field boundary treatment is used which allows waves to exit the numerical domain with very little spurious reflection.

Numerical experiments are performed which demonstrate the utility of the high-accuracy schemes using Cartesian and curvilinear grids. Two schemes are studied, one which produces the maximum formal order of accuracy, and one which is optimized for propagation distances less than roughly three hundred wavelengths. The high-accuracy schemes are shown to be substantially more efficient, in both computing time and memory, than methods which are second- and fourth-order in space. The optimized scheme can produce significant error reduction relative to the maximum-order scheme, with no additional expense, especially when the number of wavelengths of travel is large.
\end{abstract}




\section{Acknowledgments}

I would like to thank my supervisor, Professor Zingg, for his very helpful suggestions and guidance. I am indebted to Steve Hulshoff and Mike Sullivan for keeping the network of workstations on which my research depended up and running. Financial support for my research was provided for four years by a Natural Science and Engineering Research Council of Canada Postgraduate Scholarship, and for four terms by a University of Toronto Open Doctoral Fellowship. I would also like to thank my family for supporting my efforts in continuing my education. Most importantly, I would like to thank my wife Kim for keeping me sane these last few years and always managing to be enthusiastic about my still being in school. 


\section{CONTENTS}

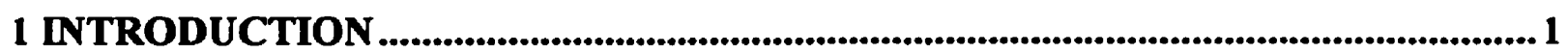

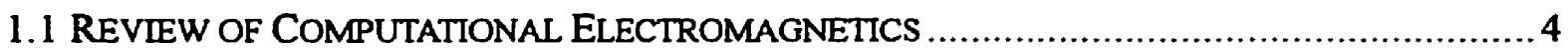

1.2 REVIEW OF HIGH-ACCURACY FINTIE-DIFFERENCE METHODS................................ 12

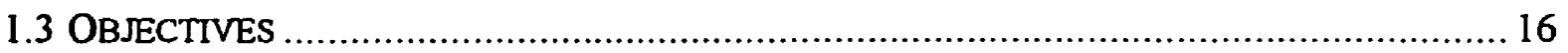

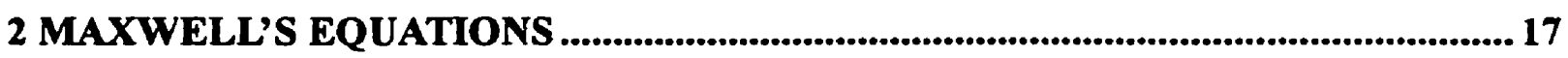

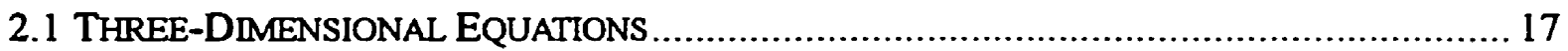

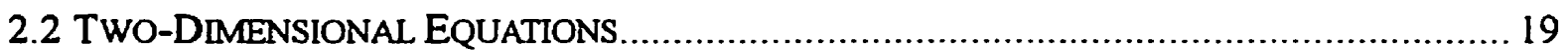

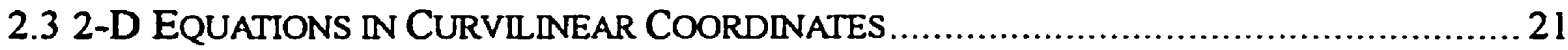

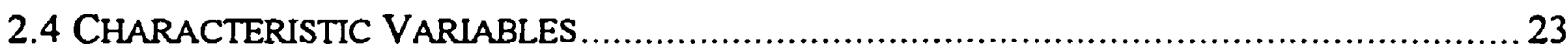

2.4. I Characteristic Variables in Cartesian Coordinates............................................. 23

2.4.2 Characteristic Variables in Generalized Coordinates........................................ 26

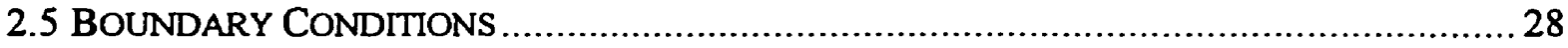

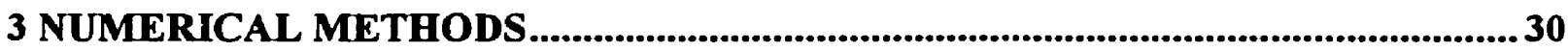

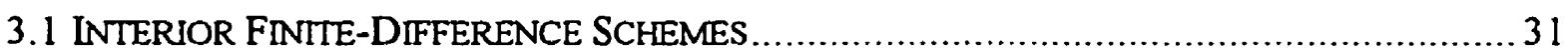

3.1.I Approximation of Maxwell's Equations.................................................................. 34

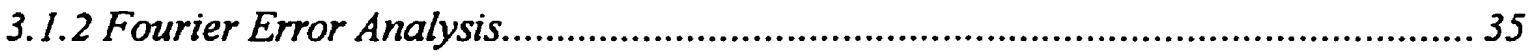

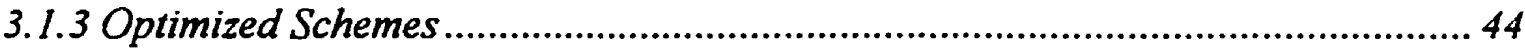

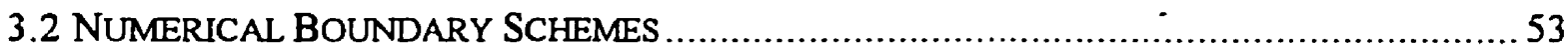

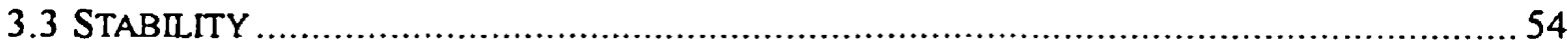

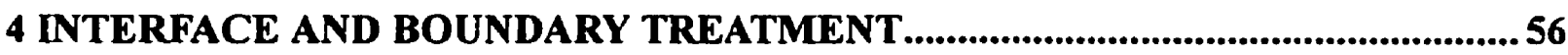

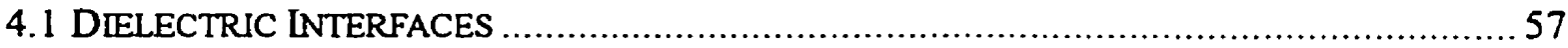

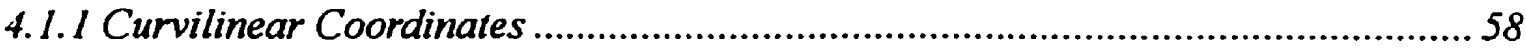

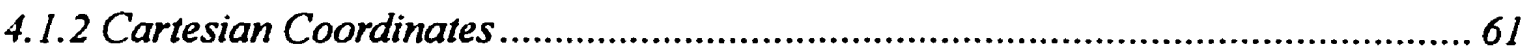

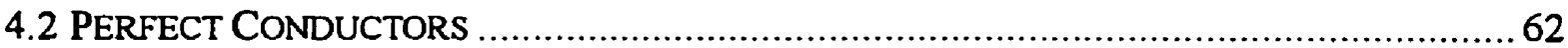

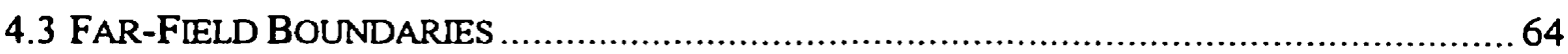

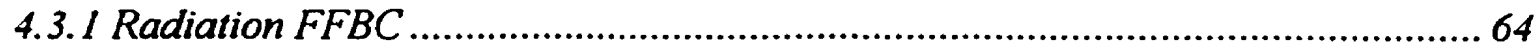




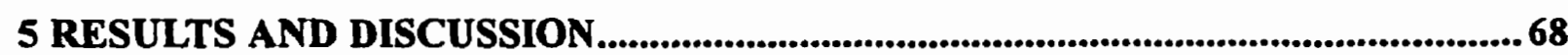

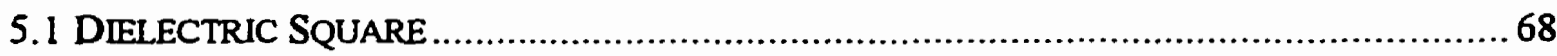

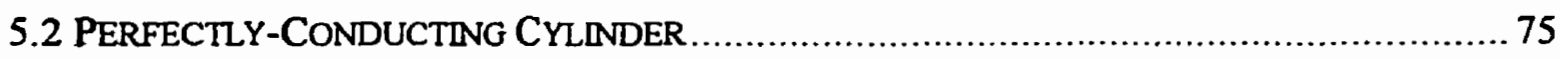

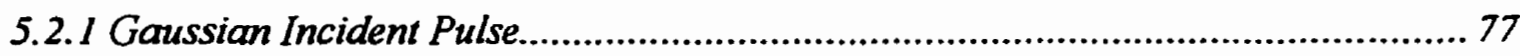

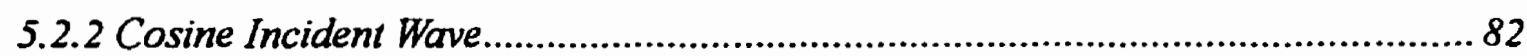

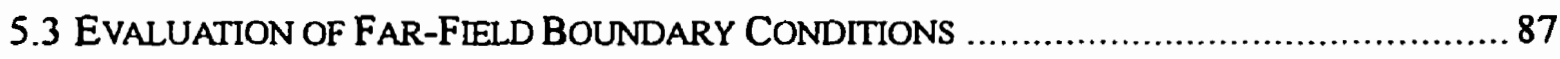

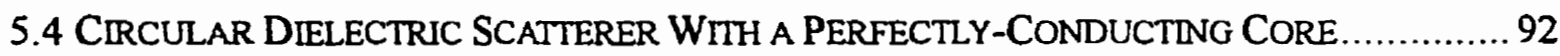

6 CONCLUSIONS AND RECOMMENDATIONS ...........................................................96

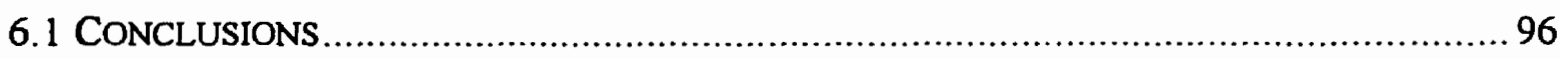

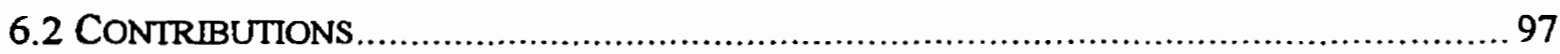

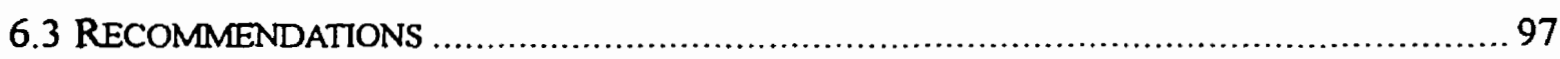




\section{List of Tables}

Table 1: Coefficients for families of central-difference operators given by equation ( 3.1 ) ....33

Table 2: Coefficients for families of filter operators given by equation ( 3.2 )................. 33

Table 3: Grids used for dielectric square tests ................................................. 70

Table 4: Grids used for perfectly-conducting cylinder tests, where resolutions are given

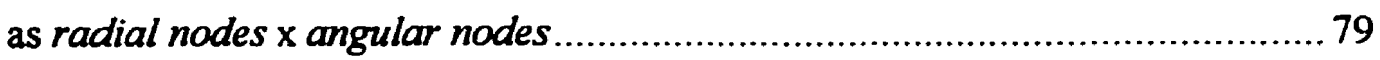

Table 5: Coefficients of optimized finite-difference schemes ..................................... 103 


\section{List of Figures}

Figure 1: a) $d$ and $d s$ on an open surface, b) $d s$ on a closed surface ............................ 18

Figure 2: Surface currents and charge density on material interface ...................................28

Figure 3: Interior, $\mathbf{i}$, and boundary, $\mathbf{b}$, nodes near corner of domain for a seven point

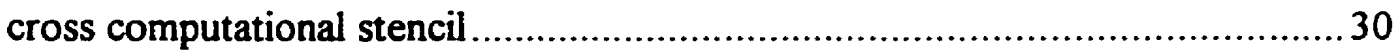

Figure 4: Example of harmonic signal resolved by a grid using 8 PPW ............................. 37

Figure 5: Local amplitude errors with $\theta=0^{\circ}$ and $C=1$ for second-order (C2), fourthorder (C4), and sixth-order (MO) schemes

Figure 6: Normalized local phase errors with $\theta=0^{\circ}$ and $C=1$ for second-order (C2), fourth-order (C4), and sixth-order (MO) schemes

Figure 7: PPW required to propagate a wave $n_{w}$ wavelengths while keeping the global errors less than 0.1 with $\theta=0^{\circ}$ and $C=1$ for second-order (C2), fourthorder (C4), and sixth-order (MO) schemes

Figure 8: Errors for scheme optimized for $\theta=0^{\circ}$ and $\left.C=1, a\right)$ and b) amplitude and phase errors for $\theta=0^{\circ}$ and $C=1, \mathrm{c}$ ) and d) amplitude and phase errors for $\theta=45^{\circ}$ and $\left.C=1, \mathrm{e}\right)$ and $\left.\mathrm{f}\right)$ amplitude and phase errors for $\theta=0^{\circ}$ and $C=1 / 2$

Figure 9: Local amplitude errors with $\theta=0^{\circ}$ and $C=1$ for MO, O10, 07.5, and O5 schemes.

Figure 10: Normalized local phase errors with $\theta=0^{\circ}$ and $C=1$ for MO, O10, O7.5, and O5 schemes.

Figure 11: PPW required to propagate a wave $n_{w}$ wavelengths while keeping the global errors less than 0.1 with $\theta=0^{\circ}$ and $C=1$ for MO, O10, 07.5, and 05 schemes.

Figure 12: Local amplitude errors for $\mathrm{O} 10$ scheme with different values of $\theta$ at a) $C=1$ and b) $C=1 / 2$.

Figure 13: Normalized local phase errors for $\mathrm{O} 10$ scheme with different values of $\theta$ at

a) $C=1$ and b) $C=1 / 2$.

Figure 14: Alignment of Numerical Domains (ND) with Dielectric Materials. 56 
Figure 15: Field values and material properties near interface

Figure 16: Example of a body fitted grid showing the characteristic variables associated with the outgoing waves. 58

Figure 17: Example of Cartesian grid with interface along line of constant $x$.

Figure 18: Example of a body fitted grid near the surface of a perfect conductor 63

Figure 19: Example of stencils used near comer of numerical domain 66

Figure 20: Fields stored near outer boundary of domain 66

Figure 21: Example grid showing pulsed plane wave with Gaussian cross-section incident at $45^{\circ}$ on a dielectric square.

Figure 22: Contour plots of electric field intensity for a) reference solution, b) MO using a grid with 11,200 nodes, c) C4 with 11,200 nodes, and d) C2 with 44,800 nodes

Figure 23: Electric field intensity across diagonal of dielectric square.

Figure 24: Normalized $L_{2}$ norm of the error in the electric field intensity, for the dielectric square case, as a function of the number of nodes in the grid

Figure 25: Normalized $L_{2}$ norm of the error in the electric field intensity, for the dielectric square case, as a function of the CPU cycles necessary to complete the simulation

Figure 26: Example grid showing a pulsed plane wave with a Gaussian cross-section incident on a perfectly-conducting cylinder

Figure 27: Transformation of physical domain into computational.domain showing different types of boundaries for a perfectly-conducting cylinder

Figure 28: Overlapping grids showing example points at which data can be passed directly, and points at which interpolation must be used

Figure 29: Contour plots of electric field intensity for a) reference solution, b) MO using a grid with 5,400 nodes, c) C4 with 5,400 nodes, and d) C2 with 21,600 nodes

Figure 30: Electric field intensity along a radial line behind the perfectly-conducting cylinder for an incident pulse with a Gaussian cross-section 
Figure 31: $\mathrm{L}_{2}$ norm of the error in the electric field intensity as a function of the number of nodes in the grid for a pulsed plane wave incident on the perfectlyconducting cylinder

Figure 32: $L_{2}$ norm of the error in the electric field intensity as a function of the number of CPU cycles it takes to complete the simulation for a pulsed plane wave incident on the perfectly-conducting cylinder

Figure 33: Electric field intensity for cosine wave, with $\kappa=2 \pi$, incident on a perfectlyconducting cylinder having radius of one

Figure 34: $L_{2}$ norm of the error in the electric field intensity as a function of the number of nodes in the grid for a cosine wave, with $\kappa=2 \pi$, incident on the perfectly-conducting cylinder.

Figure 35: $L_{2}$ norm of the error in the electric field intensity as a function of the CPU time necessary to complete the simulation for a cosine wave, with $\kappa=2 \pi$, incident on the perfectly-conducting cylinder.

Figure 36: Electric field intensity along a radial line behind the perfectly-conducting cylinder for an incident cosine wave with $\kappa=2 \pi$

Figure 37: Electric field intensity along a radial line behind the perfectly-conducting cylinder for an incident cosine wave with $\kappa=8 \pi$

Figure 38: Electric field intensity along a radial line behind the perfectly-conducting cylinder for an incident cosine wave with $\kappa=10 \pi$.

Figure 39: Geometry used to test different far-field boundary conditions in Cartesian coordinates.

Figure 40: $L_{2}$ norm of the electric field intensity as a function of the time elapsed during the simulation, for a pulsed plane wave moving through a square domain, using different far-field boundary conditions

Figure 41: $L_{2}$ norm of the electric field intensity as a function of the time elapsed during the simulation, for a pulsed plane wave incident on a perfectly conducting cylinder, using different far-field boundary conditions 
Figure 42: $L_{2}$ norm of the error in the electric field intensity as a function of the number of nodes in the grid, for a cosine wave, with $\kappa=2 \pi$, incident on the perfectly-conducting cylinder, using different far-field boundary conditions

Figure 43: Electric field intensity along a radial line behind the perfectly-conducting cylinder, for an incident cosine wave with $\kappa=2 \pi$, using different far-field boundary conditions

Figure 44: Example grid showing a pulsed plane wave incident on a circular dielectric scatterer with a perfectly-conducting core .93

Figure 45: Transformation of physical domain into computational domain, showing different types of boundaries for a circular dielectric scatterer with a perfectly-conducting core

Figure 46: Electric field intensity for Gaussian pulse incident on a circular dielectric scatterer with a perfectly-conducting core

Figure 47: Nodes, $*$, on outside surface of dielectric at which $w_{+}$must be interpolated 104

Figure 48: Data needed to interpolate $B_{n}$ onto the interface for a) a node located on both sides of the interface and b) a node located only on the dielectric side of the interface.

Figure 49: TM harmonic wave incident on a perfectly-conducting circular cylinder of radius $a$ 


\section{List of Appendices}

Appendix A: Obtaining the Strong Conservation Law Form of Equations ..........................99

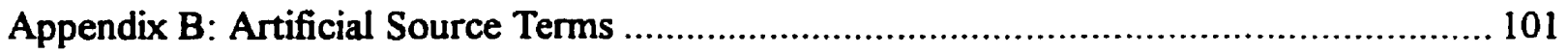

Appendix C: Coefficients of Optimized Finite-Difference Schemes................................. 103

Appendix D: Dielectric Square - Interpolation and Corners.......................................... 104

Appendix E: Analytical Solution for a Harmonic Electromagnetic Wave Incident on a

Perfectly-Conducting Circular Cylinder ..................................................... 107 


\section{Nomenclature}

A, B flux Jacobians

$\hat{\mathbf{A}}, \hat{\mathbf{B}}$ flux Jacobians in generalized coordinates

$A^{ \pm} \quad$ split flux Jacobians

B magnetic flux density

$C=c \frac{\Delta t}{\Delta x}$, Courant number

D electric flux density

E electric field intensity

$E_{n}, E_{t}$ normal and tangential components of electric field intensity

$E_{x} \quad x$ component of electric field intensity

$E^{\text {Ref }}$ reference value for electric field intensity

$E^{(1)} \quad$ electric field intensity in region (1)

$E r_{a} \quad$ global amplitude error

$E r_{p} \quad$ global phase error

F, $\mathbf{G}$ flux vectors

$\hat{\mathbf{F}}, \hat{\mathbf{G}}$ flux vectors in generalized coordinates

$\mathbf{F}^{ \pm} \quad$ split flux vectors

H magnetic field intensity

J electric current density

$J$ Jacobian of coordinate transformation

$J_{s} \quad$ surface current

$\mathrm{O}($ ) order of approximation
Q vector of dependent variables

Q dependent variables in generalized coordinates

$\mathbf{X}_{\boldsymbol{A}}$ matrix of right eigenvectors of $\mathbf{A}$

a attenuation coefficient of radiating boundary condition

$a_{i} \quad$ coefficients of central-difference operator

$c \quad=\frac{1}{\sqrt{\varepsilon \mu}}$, speed of light in medium

$d_{i} \quad$ coefficients of filter operator

$e$ base of natural logarithm

$e r_{a} \quad$ local amplitude error

$e r_{p} \quad$ normalized local phase error

$e r_{a}^{\text {sd }} \quad$ semi-discrete local amplitude error

$h, \Delta t$ time-step

$j=\sqrt{-1}$, imaginary number

n vector normal to surface

$n_{\lambda} \quad$ number of points per wavelength

$n_{t} \quad$ number of time-steps

$n_{w} \quad$ number of wavelengths

$q_{v} \quad$ electric charge density

$u_{i} \quad$ dependent variable at the $i^{\text {th }}$ node

$u_{n} \quad$ dependent variable at $n^{\text {th }}$ time-step

$u_{n+a_{1}}^{(1)}$ dependent variable at first stage of time-marching method 
$w_{+}, w$. characteristic variables

$z \quad=\kappa \Delta x$, nondimensional wavenumber

$z_{1 \mathrm{D}}^{\bullet}$ one-dimensional modified nondimensional wavenumber

$z_{2 \mathrm{D}}^{*}$ two-dimensional modified nondimensional wavenumber

$\nabla \quad$ gradient operator

$\nabla \times \quad$ curl operator

$\nabla \bullet \quad$ divergence operator

$\Delta x \quad$ grid spacing in $x$ direction

$\Lambda_{A}$ diagonal matrix of eigenvalues of $A$

$\alpha, \beta$ coefficients of inflow NBS

$\alpha_{i}$ coefficients of time-marching scheme

$\beta_{i} \quad$ coefficients of characteristic root

$\delta_{x}^{a} \quad$ antisymmetric or central-difference operator

$\delta_{x}^{s} \quad$ symmetric or filter operator

$\varepsilon \quad$ electrical permittivity

$\eta_{l}=\sqrt{\frac{\mu}{\varepsilon}}$, intrinsic impedance of medium

$\kappa \quad$ spatial wavenumber

$\kappa^{*}, z^{*}$ modified wavenumber and nondimensional wavenumber

$\lambda$ coefficient of representative ODE $\lambda_{w} \quad$ wavelength

$\mu \quad$ magnetic permeability

$\theta$ angle

$\rho_{s} \quad$ surface charge density

$\sigma \quad$ conductivity

$\sigma \quad$ standard deviation of Gaussian distribution

$\sigma \quad$ characteristic root of time-marching scheme

$\sigma_{r}, \sigma_{t}$ real and imaginary parts of characteristic root

$\omega=c \kappa$, angular frequency

$\xi, \eta \quad$ computational coordinates 


\section{Introduction}

Many physical systems give rise to wave-like phenomena. For example, the elastodynamic equations which describe the internal motion of elastic materials, the acoustic equations which describe the properties of small disturbances in a fluid, and Maxwell's equations which describe the time evolution of electromagnetic fields, all produce solutions with wave-like properties. Analytical solutions for these equations are available only for problems which have very simple geometries. As a result, the modeling of these equations using computational methods has led to the creation of several new fields including computational geophysics, computational aeroacoustics, and computational electromagnetics. A common problem in all these fields is how to model accurately the propagation of linear waves over long distances, and how to simulate their interaction with complex scattering geometries.

Current developments in the defense, electronics, and medical industries have increased the importance of finding accurate solutions to scattering problems in electromagnetics. A number of important applications are given by Taflove [72]. Defense applications include radar cross-section modeling of aircraft, and the optimization of both the materials used in the manufacturing of components and component shapes, in order to produce stealthy aircraft. The design of complex phased-array radar systems and accurate target identification are also important applications for the defense industry. Two important medical applications are modeling the physics of human vision, and simulating electromagnetic wave interaction with the human body in order to aid in designing protocols for hyperthermia treatment of cancer. Simulations are used in the electronics industry to help design digital circuit packaging and interconnects, as well as subpicosecond photonic devices such as ultra-high-speed photonic switches, and to model cross-talk effects in circuit boards.

Frequency-domain methods for solving Maxwell's equations, such as the method of moments, have enjoyed significant popularity in the electrical engineering community. Scaling arguments have been presented $[72,78]$ which show that time-domain methods are better suited for the simulation of scatterers whose size is large compared to the wavelength of the incident wave. It has been noted by researchers in computational electromagnetics [72], computational geophysics [26], and computational aeroacoustics [44] that the second-order 
methods now enjoying the most significant use require excessively fine grids for simulating long-range wave propagation. There are a number of scattering problems which can not be solved, due to memory and time limitations, using these second-order methods and the supercomputers currently available. An example of such a problem in computational electromagnetics is radar cross section modeling of an entire aircraft for an incident wave having a frequency measured in the tens of $\mathrm{GHz}$ [72].

Due to the limitations of second-order methods, researchers have shown a renewed interest in high-accuracy schemes for solving linear wave propagation problems. Classical highorder finite-difference schemes have been used to solve high-frequency problems in many of the above mentioned fields $[9,10,11,29,57,58,87]$. In the last ten years many researchers have noted that the classic high-accuracy finite-difference schemes, which maximize the formal order by minimizing the truncation errors, often are not the best choice for solving wave propagation problems. Optimized finite-difference schemes can be developed which give significantly smaller dissipation and dispersion errors, for a given frequency range, than schemes with the same stencil which have the highest possible order of accuracy (see, for example, [15, $18,26,42,44,73,74,95])$. Since the optimized and maximum-order schemes have the same computational stencil, the accuracy improvement is obtained without requiring any additional computer resources. These optimized schemes can also give the same level of accuracy as the maximum-order schemes while using a much coarser grid. This results in a significant saving in terms of the number of arithmetic operations needed and the computer memory required, especially when modeling problems in three dimensions.

The generation of stable and accurate numerical boundary formulations is a problem that must be addressed when simulating wave scattering using these high-accuracy methods. An easy way to obtain a stable numerical method is to use low-order operators near interfaces and boundaries. This can have a significant impact on the overall error, which wastes the properties of the optimized interior schemes. A number of high-order numerical boundary schemes that can be used with high-accuracy methods are given in $[6,95]$. When scattered waves exit the numerical domain, which must be truncated in order to model a problem having an infinite extent, spurious reflections are often generated. The development of accurate absorbing/radiating boundary conditions, which limit the effects of these reflections, has been the 
focus of considerable research. Taflove [72] has stated that many of these far-field boundary conditions are the major contributor to the global error accumulated during electromagnetic simulations. Recently, a new absorbing boundary condition, for use in electromagnetic simulations, has been proposed by Berenger [3]. This method, which uses a perfectly matched layer, is very well suited for use with high-accuracy schemes.

Another important aspect when simulating scattering problems is the type of gridding method used. The simplest gridding strategy is to use regular Cartesian meshes throughout the domain. Special boundary conditions are needed when modeling curved objects with a Cartesian grid. By gradually changing the material properties near the interface between objects [83], a Cartesian grid can be used throughout the domain, and special boundary operators can be avoided. This works fairly well when using low-order operators, but the approximation of the interface generates too much error when high-accuracy methods are used. Another method uses a Cartesian grid up to cells bordering the scatterer, which are warped in order to conform to its boundary. The contour path method of Jurgens et al. [31] uses this type of grid to model electromagnetic scatterers, but it is difficult to extend this method for use with highaccuracy schemes. The use of body-fitted curvilinear grids solves the problem of modeling curved scatterers, but there is a significant increase in computer resources required, due to the need of grid generation software and the calculation and storage of grid metrics. A hybrid method that uses a body fitted grid near the boundary of the scatterer and a Cartesian grid everywhere else is given in [85]. This method is also difficult to extend to high-accuracy schemes because of the errors introduced by the extensive interpolation of data needed to pass information between grids. Unstructured grids are also ideal for use with oddly shaped scatterers, but it is quite difficult to obtain high-accuracy methods on such a grid. There is no clear way of deciding the best gridding method, since the optimum choice will be problem dependent.

This dissertation presents research concerned with the development of optimized highaccuracy finite-difference schemes for simulating linear wave propagation and their use in finding solutions for two-dimensional electromagnetic scattering problems. The rest of this chapter includes an overview of the methods used in computational electromagnetics, followed by an extensive review of high-accuracy finite-difference schemes, and it ends with the 
presentation of the objectives of this research. The next chapter focuses on the equations that govern electromagnetic wave propagation. The 3-D time-domain Maxwell's equations are presented, followed by a simplification to the 2-D set of equations. A generalization of the 2-D transverse magnetic equations to a curvilinear coordinate system is given. This is followed by a chapter that presents the numerical methods, along with an extensive Fourier error analysis. A procedure for developing finite-difference operators that generate optimal phase and amplitude errors for a given range of frequencies is presented, and a number of schemes optimized for different frequency ranges are given. Stable high-accuracy numerical boundary schemes for use with these operators are also supplied. The treatment of interfaces and boundaries is the focus of the next chapter, and methods for treating the interface between two dielectrics, and the interface between a dielectric and a perfect conductor are developed. Two far-field boundary conditions are also supplied. The next chapter discusses a number of numerical simulations in which the high-accuracy schemes are used. The simulations include the scattering of a pulsed plane wave incident on a dielectric square using a Cartesian grid, the scattering of pulsed and harmonic waves incident on a circular cylinder using a curvilinear grid, and the scattering of a pulsed plane wave incident on a circular dielectric cylinder with a perfectly conducting core. The efficiency of both an optimized and a maximum-order scheme is compared with classical second- and fourth-order methods, and the different far-field boundary conditions are evaluated. The dissertation ends with some concluding remarks, which include the contributions of this research and recommendations for further directions of study.

\subsection{Review of Computational Electromagnetics}

The equations governing electromagnetic wave propagation have been known since before the turn of the century. As pointed out by Umashankar and Taflove [78], it was not until the early 1960s, when computers became generally available, that the use of computational methods for finding solutions to Maxwell's equations became popular. In the past thirty-five years there has been much research aimed at finding new and better ways to obtain solutions for problems in electricity and magnetism. The above authors present the following major types of methods for finding numerical solutions to problems where electromagnetic waves 
interact with structures: high-frequency diffraction theory, frequency-domain integral equation formulations using the method of moments, frequency-domain differential equation formulations using finite-element methods, and time-domain differential equation formulations using finite-element, finite-difference, or finite-volume methods.

The most popular method stemming from high-frequency diffraction theory is the geometrical theory of diffraction (GTD) formulated by Keller in the 1950s [33]. Keller extended a general high-frequency theory of diffraction, that stated that an electromagnetic field can be calculated for each point on a geometrical optics ray, by adding the ability to include some more general cases. For example, he worked out the fields arising from rays diffracting off vertices and corners, and he determined a number of different features of the fields such as diffraction coefficients and decay exponents. Since Keller's original formulation, a significant amount of effort has concentrated on alleviating some of the deficiencies of GTD. Among those mentioned in [78] are: that GTD results in nonphysical infinities in the fields for certain types of geometries, it is difficult to obtain diffraction coefficients for dielectric edges and surfaces, and it is very hard to apply GTD in cavities and waveguides.

In the 1960s, Harrington came up with a method for solving the frequency-domain integral equation formulation of Maxwell's equations which he called the method of moments (MOM). An excellent reference for this method is found in his text, originally published in 1968 [20]. The MOM is based on writing the integral equations relating to a given problem as an infinite-dimensional linear operator equation where the unknown function relates to a physical quantity such as the induced currents on the surface of a scatterer. The unknown function in this equation is mapped to a finite-dimensional subspace by choosing an appropriate set of basis functions. By taking inner products of the functional expansion with another appropriate set of weighting functions, and noting that the inner products are linear, a finite set of equations is obtained whose solution gives the unknown coefficients of the basis functions. The solution of these equations results in either an exact or an approximate solution to the original unknown function, depending on the choice of the set of basis functions. The MOM has been successfully used for finding the solution to a large range of problems. Harrington's text illustrates a number of successful applications, and the object of the work 
presented by Umashankar and Taflove [78] is the "...rigorous validation..." of the MOM for a number of different types of problems.

The difficulties associated with the MOM are not due to the type of structures that can be modeled, as in the GTD, but in the computer resources required when considering structures that are electrically large. Electrically large structures are those structures whose size is measured in tens, or even hundreds, of wavelengths, where the wavelength is a measure of the incident electromagnetic field. The equations resulting from the use of the MOM give dense, full coefficient matrices, as noted in $[72,78]$. If $N$ is the number of field unknowns the MOM requires computer storage on the order of $N^{2}$, and the computer time needed to invert the matrix is on the order of $N^{2}$ to $N^{3}$. Taflove [72] states that if the size of a three-dimensional flat target is on the order of four wavelengths, $N$ will be larger than 10,000 , which is a problem size easily handled by today's computers. If the size of the object rises to thirty-two wavelengths, $N$ rises to 640,000 , and he projects that a typical supercomputer would need $10^{12}$ words of computer storage and would take almost a year and a half to obtain a solution using the MOM. In order to alleviate some of the problems associated with inverting the matrix, a number of researchers started applying iterative techniques to solve the set of equations; see, for example, [54]. These formulations led to a substantial reduction in the computer requirements, but the robustness of the method was affected, because it is often difficult to obtain a converged solution when complex structures are modeled. Current research aims at preconditioning the matrices in order to cbtain better convergence results.

The solution to electromagnetic problems using the finite-element method was first formulated for frequency-domain representations of the governing equations. The text written by Silvester and Ferrari [68] gives a good introduction to the subject. The finite-element method is based on approximating the continuous electromagnetic fields by basis functions defined on elements whose shape is dependent on the dimension of the problem. Triangular elements are popular in two dimensions and tetrahedral elements enjoy extensive use in three dimensions. The functions are defined such that they have a value of one at a given node, and are zero at every other node. A variational method can be used to find the coefficients of the basis functions, resulting in an approximation for the electromagnetic fields. The use of triangular elements for finding solutions to electromagnetic problems started in the late 1960 s; see, for 
example, [65]. The extensive tabulation of universal matrices had a significant impact on the widespread adoption of triangular elements, formulated in the so called simplex coordinates, for the solution of the frequency-domain Helmholtz equation. In 1973, Konrad and Silvester presented matrices for the axisymmetric case [36], and in 1978, Silvester published a general method for developing universal matrices [67]. Tetrahedral finite-elements, for the solution of the three-dimensional Helmholtz equation, are given in [66]. An example of the use of finiteelements for the solution of the three-dimensional Helmholtz equation can be found in [23].

The method described above is quite flexible, as many complex scattering objects can be successfully modeled. As mentioned in [72], the finite-element method will result in a system of equations which requires an inversion of a sparse matrix. Using proper grid optimizations and coordinate storage, as discussed in [68], the resulting matrix can be banded as well. Because of this, the computer resources required are much smaller than those demanded by the MOM, where the matrix is quite dense. Thus the method can scale quite well when considering electrically large objects. A drawback that Umashankar and Taflove point out [78] is that the computer resources required by any finite-element solver are heavily dependent on the mesh used and the coordinate storage. Generally, when considering a solution in the frequency-domain some flexibility is lost over a pure time-domain approach. Shankar et al. [63] note that a time-domain-based method can handle both a single frequency and a broadband pulse incident field. A frequency-domain method can only provide a solution for a single frequency while, through the use of Fourier transforms, a time-domain solution can provide information about multiple frequencies from a single broadband pulse. Also, a time-domain based method can easily handle active surfaces made up of materials whose properties vary in time.

The direct solution of Maxwell's time-domain curl equations has been presented by many researchers as a solution to the above mentioned limitations. There are two main steps in solving the time-domain equations. First, the computational domain must be spatially discretized using some type of mesh, and finite-differences, finite-elements, or finite-volumes can be used, in conjunction with the governing equations, to obtain the time variation of the fields at a given instant in time. With this information, a time-marching method can be used to determine the fields at a later instant in time. Repeating these two steps, for as long as required, 
allows the simulation of the dynamics of the electromagnetic fields throughout the computational domain.

The use of finite-elements to solve the time-domain equations has been studied by, for example, Cangellaris et al. [5], Petitjean et al. [53], and Feliziani and Maradei [13]. As mentioned above, the finite-element method results in the need for a banded or sparse matrix to be inverted. If the mesh is not time dependent, this matrix has to be inverted only once, at the beginning of the simulation. The overall impact this inversion has on the CPU time needed to complete the simulation depends on the ratio of the number of elements in the spatial domain to the total number of time-steps. If the simulation is run for many time-steps, the overhead in the inversion may be negligible. The storage needed for this matrix can be quite significant when electrically large structures are being modeled. The need for the storage and inversion of a matrix can be avoided through the use of finite-volumes or finite-differences, provided that non-compact spatial schemes are used and the time-marching method is explicit.

The finite-volume time-domain method has been used to model many problems in electromagnetics by Shankar and his collaborators [47, 59-63]. Other researchers using the finitevolume time-domain method include Madsen and Ziolkowski [45], Bishop and Anderson [4], Noack and Anderson [51], and Shang et al. [57, 58]. Yee and Chen [86] have also presented a hybrid finite-difference/finite-volume time-domain method. A finite-volume method developed for computational fluid dynamics is used in the solver produced by Shankar's group. The first step in applying this method to electromagnetic problems is to write Maxwell's time-domain equations in a conservation-law form. To handle complex geometries they use a body-fitted mesh, and a general coordinate transformation is applied in order to map the governing equations into a simpler computational space. The fields are stored at each node in the mesh and are taken to represent the average value of the fields in a small volume, or cell, centered at this node. The time variation of these fields is calculated numerically using the conservation law, which can be stated in the following way: the rate of increase in the fields inside a cell (the quantity being conserved) is equal to the fluxes entering through the outer surfaces of the cell plus any contributions due to sources inside this cell. A characteristic-based Riemann solver is used in order to evaluate the fluxes along cell boundaries, and the sources are usually simple functions of the fields inside the cells. An explicit Lax-Wendroff two-step (predictor- 
corrector) scheme is used to march the fields through time. In [63], Shankar et al. present an extensive discussion of the flexibility of this computer program, and include a number of examples of its use in modeling problems with very complex geometries. They also discuss planned development for their solver, an important aspect being the introduction of higherorder methods which would allow the use of coarser grids. This would enable the modeling of larger problems using the computer resources currently available.

According to Shlager and Schneider [64], the “... Finite-Difference Time-Domain (FDTD) method is arguably the most popular numerical method for the solution of problems in electromagnetics." In their paper, they present a selective survey of the research presented in the literature on the FDTD method. Another extremely good reference on the FDTD method is the text written by Taflove [72]. It is generally accepted that the use of the FDTD method to solve Maxwell's curl equations was first proposed by Yee [84]. His original method uses staggered regular Cartesian grids, with the components of the electric and magnetic fields stored in an uncolocated manner [43]. The electric and magnetic fields are also staggered in time with a separation of a half time-step. An explicit leap-frog time-marching method is used to propagate the fields through time. Both the spatial and time derivatives in the curl equations are approximated by second-order central-difference schemes. There are a number of problems associated with Yee's original formulation, as pointed out in $[72,78]$. Some of the problems with the original formulation are that it lacked a far-field boundary condition which would allow the scattered fields to exit the numerical domain without spurious reflections, there was no way of modeling wires and slots which had dimensions less than the size of a cell, and the outer boundaries of scatterers had to be modeled using a stepped or staircased approximation. These problems have been addressed in the literature, and the FDTD method is now quite robust and is being used to solve many different types of problems [64, 72].

Most finite-difference methods that solve Maxwell's time-domain equations, discussed in the literature, are based on Yee's method, using staggered grids and a leap-frog timemarching method. A number of researchers have presented schemes which use different gridding arrangements, spatial differencing, and time-marching methods. Many of these solvers have borrowed heavily from the field of computational fluid dynamics, using ideas such as upwind schemes, body-fitted coordinate systems, and the finite-volume methods discussed 
above. Yarrow et al. [83] present a method which uses a single grid, and they consider second and fourth-order centered and upwind finite-differences for the spatial derivatives in conjunction with a fourth-order Runge-Kutta time-marching method. An analysis of the errors associated with different types of spatial gridding methods, such as staggered and unstaggered Cartesian and hexagonal grids, spatial differencing schemes, including centered and upwind methods, as well as time-marching methods, such as staggered and unstaggered leap-frog and third and fourth-order Runge-Kutta methods, is presented by Liu [43].

In order to handle more complex geometries, a number of methods were introduced in an effort to reduce the errors inherent in using staircasing near curved objects, and to allow the modeling of small subcell structures. Zivanovic et al. [96] present a subgridding method which allows a fine mesh to be imbedded inside a coarser mesh. Holland [27] and Fusco et al. [14] use a curvilinear coordinate formulation which can be body-fitted to handle curved bodies. Shang and Fithen [56] give a characteristic method which uses upwind finite-differences in curvilinear coordinates. A method which incorporates a body-fitted grid which overlaps a regular mesh is presented by Yee et al. [85]. The contour-path method was used by Umashankar et al. [77] to model small wires, by Taflove et al. [70] to model narrow slots, and was extended by Jurgens et al. [31] to handle curved surfaces. Madsen [46] introduced a method closely related to the FDTD method called the discrete surface integral method which uses unstructured grids. The finite-surface method was introduced by Vinokur and Yarrow $[80,81]$. The method is applied directly to the integral Faraday's and Ampère's laws, and uses surface integrals as the unknowns. Generalized non-orthogonal coordinates are used, and the method can handle smooth interfaces and different types of singularities.

The use of special far-field boundary conditions which reduce the errors introduced by spurious reflections as the scattered fields exit the computational domain was initiated by the pioneering work presented by Engquist and Majda [12] and Bayliss and Turkel [2]. Both of these works introduced special differential equations which were applied at the outer boundary of the domain. Engquist and Majda presented absorbing boundary conditions (ABCs) which are based on Padé approximations to a one-way wave equation. Bayliss and Turkel introduced a radiation boundary condition (RBC) which only allows the fields near the outer boundaries to propagate in the outward direction. In 1981 Mur [49] introduced a second- 
order accurate ABC for the Yee algorithm. In 1987, the work presented by Higdon [22] greatly improved upon the ideas originally put forth by Engquist and Majda. He presented a simple method for generating an $\mathrm{ABC}$ of arbitrary order which produces zero reflections for waves exiting the domain at a number of different discrete angles. At around the same time, Kriegsmann et al. [39] and Moore et al. [48] published articles which introduced the electromagnetics community to the general theory of ABCs and RBCs. A number of methods which were originally formulated for problems in computational aeroacoustics prove equally useful when modeling Maxwell's equations. Thompson [75, 76] presented a method which is based on a locally one-dimensional characteristic splitting, Giles [16] introduced a method which limits the propagation of waves with group velocities pointed into the numerical domain, and Tam and Webb [73] give a set of boundary conditions developed by performing an asymptotic analysis of the Euler equations. An evaluation of these three methods was performed by Hixon et al. [25], and he concluded that Tam and Webb's method was the most accurate. For problems with no mean flow, which results in a set of equations which describe the same physics as Maxwell's equations, Tam's method is identical to the one presented by Bayliss and Turkel.

Taflove [72] noted that the spurious reflections resulting from the use of the $A B C s$ and RBCs described in the above paragraph were becoming the limiting errors in many of the calculations performed using the FDTD method. This explains the importance, and popularity, of the $A B C$ presented by Berenger [3] which results in reflection coefficients that are two orders of magnitude smaller than those given by previously used methods. He introduces an absorbing material, in which propagating waves are attenuated exponentially, that can be matched to other materials in such a way that a wave will enter into it, at any angle of incidence, without producing any reflections. This perfectly matched layer (PML) surrounds the computational domain and a perfectly-conducting boundary condition is imposed on the outside of the PML. When a scattered wave exits the computational domain it enters into the PML without any spurious reflections, it travels through the PML and reflects off of its outer boundary, causing it to travel back toward the computational domain. While inside the PML the wave is attenuated exponentially at a rate which can be set in order to achieve a desired level of reflection. Berenger's method was originally presented for two-dimensional Cartesian coordinates, but it was quickly extended to more general cases. Katz et al. [32] extend the method to three 
dimensions for the Yee FDTD method, Chew and Weedon [8] apply the method to the 3D Maxwell's equations with stretched coordinates, and Navarro et al. [50] and Wu et al. [82] use PMLs with a nonorthogonal FDTD method.

Over the last thirty years the FDTD methods have become extremely robust. They are capable of handling a very large range of problems involving scattering from complex geometries. The computer resources required by the finite-volume and finite-difference time-domain solvers are much better than those required by the MOM. For example, Taflove shows [72] that for three-dimensional problems the computer time needed to run a simulation using these methods scales as $N^{4 / 3}$, while the original MOM scales as $N^{3}$. It can be argued that the MOM will have a value of $N$ that is much smaller than that required by the FDTD method because cells are only required on the surface of the scatterer, while the FDTD mesh permeates the entire volume of the problem. This may be the case for simple problems, but for many of the complex geometries now being modeled by electrical engineers, volumetric MOM approaches must be used [78]. Although current FDTD algorithms are among the most efficient methods in use today, Taflove [71, 72] points out the need for methods with higher accuracy. By increasing the formal order of current solvers, problems of much larger electrical size can be modeled, and much coarser grids can be used, giving a substantial savings in terms of the time needed to complete a simulation and the computer storage required. For electrically large problems, the use of the PML ABC has caused a shift in where the bulk of the error is generated during a simulation. The major error contribution used to be from spurious reflections caused by the $\mathrm{ABC}$, but in many cases it is now due to the numerical-phase errors caused by the interior finite-difference schemes.

\subsection{Review of High-Accuracy Finite-Difference Methods}

High-accuracy finite-difference schemes for approximating spatial derivatives and performing time integration have been available for many years. Kopal [37] presents a number of high-accuracy schemes in his text, including various compact (Padé) schemes having up to tenth-order formal accuracy. Among the best known high-accuracy time-marching methods are the Runge-Kutta methods. A discussion of Runge-Kutta methods can be found in many texts such as [40], and many researchers have presented high-accuracy schemes with special 
properties, see for example [41]. In the last ten years, a lot of interest has been focused on the use of high-accuracy methods for the numerical simulation of wave propagation problems. A number of simulations were being run in which second-order methods are inadequate because they require an excessively fine grid in order to propagate a disturbance the required distance without the solution being polluted by numerical errors. Methods which have a high formal order have been used to model wave-like phenomena in many fields. Dablain [10] used highorder schemes to obtain the solution of the scalar wave equation. Cohen [9] used a fourthorder method for solving the acoustic equations, and the unsteady Euler equations were solved by Yu et al. [87] with a method that combines a compact sixth-order central-difference spatial operator with a fourth-order Runge-Kutta scheme. Thomas and Roe presented fullydiscrete nondissipative schemes for computational aeroacoustics which use an upwind leapfrog method on staggered meshes. A second-order version of the method is presented in [74], and a fourth-order version, which was given at the accompanying oral presentation, can be found in [92]. Recently, Castillo et al. [7] gave fourth-order approximations for the divergence, gradient, and Laplacian operators, and Olsson [52] and Strand [69] presented methods for developing stable schemes of arbitrary order for hyperbolic problems. A number of methods have been presented specifically for Maxwell's equations. For example, Deveze et al. [11] gave a fourth-order FDTD method, Jurgens and Zingg [29] used a method which combines a fifth-order spatial scheme with a sixth order Runge-Kutta scheme, and Shang et al. [57, 58] presented a method which uses a third-order finite-volume formulation with fourth-order Runge-Kutta time-marching.

A fairly new development in numerical methods is the construction of finite-difference schemes which sacrifice a high formal order of accuracy in order to obtain better error properties for waves with a given range of frequencies, as outlined by Vichnevetsky and De Schutter [79]. This is accomplished by using schemes which have large stencils that are constrained to have some low formal order of accuracy. This frees up a number of coefficients which can be used to tailor the error properties of the method. The geophysics community was the first to make extensive use of these methods. Holberg [26] analyzed a number of central-difference schemes, using 2 to 30 sampling points, which were optimized in order to constrain the error in the group velocity of the one-dimensional wave equation for the largest 
possible range of wavenumbers. He also compared the computer memory and arithmetic requirements of the schemes, as a function of the stencil size, while keeping the maximum error in the group velocity fixed. Kindelan et al. [35] extended the work of Holberg by presenting an easier method for generating the coefficients of his optimized schemes, and they compared the memory requirements and arithmetic cost of a number of schemes to a conventional highorder finite-difference scheme and a pseudospectral method. Sguazzero et al. [55] extended Holberg's work to take into account the effects of the time-marching method when modeling the 2-D and 3-D elastic equations, and developed an optimized spatial operator which uses staggered grids with leap-frog time-marching.

The 1990s have seen a significant amount of research focused on the development and application of such optimized schemes. In 1992, Lele [42] presented compact central-difference operators for approximating first, second, and third derivatives which have spectral-like resolution, but the schemes were not formally optimized in any way. In 1993, Tam and Webb [73] presented a seven-point spatial operator and a four-stage time-marching method which are optimized in order to minimize the error in the dispersion relation for the acoustic equations. Also in 1993, Zingg et al. [89] presented a number of seven-point spatial operators, and six-stage Runge-Kutta methods which were jointly optimized in order to provide schemes which minimize the phase and amplitude errors for problems in which the smallest wavelengths are resolved by a given number of grid points. A number of the optimized schemes of Zingg et al. were evaluated for use in modeling the one-dimensional form of Maxwell's equations [91], and the application of a scheme optimized for a grid with. a resolution of at least ten points-per-wavelength to two-dimensional electromagnetic scattering problems can be found in [30]. In 1994, Lockard et al. [44] gave an optimized upwind spatial scheme which uses a seven point stencil, and Haras and $\mathrm{Ta}^{\prime}$ asan [18] extended the work of Lele by generating a number of compact schemes which are optimized for propagating pulses with Gaussian cross-sections. In 1995, Taflove [72] presented an extension to Yee's FDTD method which uses a fourth-order spatial operator whose coefficients are perturbed slightly in order to produce optimized dispersion properties. In 1996, Gaitonde and Shang [15] used a method which combines an optimized compact finite-volume scheme with a classical fourth-order time marching method to solve Maxwell's equations, and Kim and Lee [34] further optimized the 
compact schemes presented by Lele. Zingg and his collaborators [92, 94] have evaluated a number of these optimized schemes and have shown that one of the compact schemes of Haras and Ta'asan requires less than four grid points per wavelength to propagate a wave 200 wavelengths, and that the noncompact schemes of Lockard et al. [44] and Zingg et al. [95] require less than ten grid points per wavelength. These noncompact schemes require far less computational effort per grid point than Haras's and Ta'asan's scheme, which requires the solution of a pentadiagonal system of equations in each coordinate direction. The relative efficiencies of these schemes still needs to be determined.

A number of researchers have placed significant emphasis on the choice of a timemarching method for use with their high-accuracy spatial operators. There is a need for the errors generated by the time-marching scheme to be of the same level as that of the spatial operators. Leapfrog methods have been used by a number of groups because of their nondissipative properties $[55,72,74]$. Many groups have optimized their spatial operators and timemarching methods separately. For example, Tam and Webb [73] use an optimized four level Adams-Bashforth type method, and Haras and Ta'asan [18] use an optimized five-stage lowstorage Runge-Kutta type method. Zingg et al. [89, 95] use a six-stage low-storage RungeKutta type method which is optimized with their spatial operator. When solving an ordinary differential equation, Adams-Bashforth methods require fewer derivative evaluations than Runge-Kutta methods of the same formal order, but they require significantly more storage because they need a number of these derivative values from previous time steps. Zingg and Lomax [90] evaluate a number of Runge-Kutta methods for use with the maximum-order high-accuracy spatial scheme presented in [89]. Additional research by Zingg and Chisholm [93] found low storage Runge-Kutta methods, and Hu et al. [28] discuss a number of optimized and low-storage schemes.

The generation of stable and accurate boundary schemes is also a topic of considerable interest for the high-accuracy finite-difference community. Some researchers, for example, [72], have suggested the use of lower-order methods near boundaries to avoid the difficulty of developing higher-order numerical boundary schemes (NBSs). The use of a low-order NBS reduces the global accuracy of the overall numerical method to be the order of the NBS plus one [6]. This can have a very significant impact on the numerical errors accumulated during a 
simulation. A number of researchers have presented stable high-order NBSs which preserve the accuracy of the interior operators. Zingg and Lomax [88] presented a fifth-order twoparameter NBS which is stable when used with sixth-order central-differencing operators. Carpenter et al. [6] developed a number of stable high-order NBSs for use with compact fourth- and sixth-order schemes, using the stability theory of Gustafsson, Kreiss and Sundström (G-K-S) [17] for the semi-discrete analysis, and the theory of Kreiss and Wu [38] to generalize to the fully-discrete case. A similar method is used by Strand [69] in order to develop schemes of general order of accuracy $2 r$. Olsson [52] proposes a method for developing stable schemes of arbitrary order by using a summation by parts rule.

\subsection{Objectives}

The objectives of this research are:

1. to develop high-accuracy finite-difference methods for simulating electromagnetic wave propagation and scattering, including schemes with optimized error properties,

2. to address implementation issues including proper material interface conditions, farfield boundary conditions, and stable and accurate numerical boundary schemes,

3. to demonstrate the high-accuracy methods' capability for solving realistic scattering problems using both Cartesian and curvilinear grids,

4. to demonstrate the numerical efficiency of the high-accuracy schemes as compared to lower-order finite-difference methods. 


\section{Maxwell's Equations}

\subsection{Three-Dimensional Equations}

In order to discuss the propagation of electromagnetic waves, the basic theory of electricity and magnetism must be understood. There are many texts which present the equations which govern the interaction of electromagnetic fields. For example, Hayt [21] has written an introductory text on the subject of electromagnetics, while Harrington [19] presents a much more advanced look at the development of electromagnetic theory for time-harmonic electromagnetic fields. Umashankar and Taflove [78] present a very detailed examination of the physics of the equations in their text which focuses on the numerical simulation of electromagnetic fields.

The most general set of equations which describes the behavior of electromagnetic fields is the integral form of Maxwell's equations:

$$
\begin{array}{ll}
\oint \mathbf{E} \cdot d \mathbf{l}=-\frac{d}{d t} \iint \mathbf{B} \cdot d \mathbf{s} & \oiint \mathbf{B} \cdot d \mathbf{s}=0 \\
\oint \mathbf{H} \cdot d \mathbf{l}=\frac{d}{d t} \iint \mathbf{D} \cdot d \mathbf{s}+\iint \mathbf{J} \cdot d \mathbf{s} & \oiint \mathbf{D} \cdot d \mathbf{s}=\iiint q_{v} d \tau
\end{array}
$$

Contained within these equations is a statement of the conservation of charge as it applies to a region:

$$
\oiint \mathbf{J} \cdot d \mathbf{s}=-\frac{d}{d t} \iiint q_{v} d \tau
$$

The above set of equations relates the following six physical quantities:

E the electric field intensity

H the magnetic field intensity

D the electric flux density

B the magnetic flux density

J the electric current density

$q_{v} \quad$ the electric charge density

All the above terms are vector quantities, except for the electric charge density which is a scalar. 
When evaluating the integrals in equations ( 2.1 ) and (2.2) a number of conventions must be noted. A circle on a line integral denotes a closed contour, and a circle on a surface integral denotes a closed surface. When a contour and surface integral appear together in an equation, the contour is assumed to wrap around the outer edge of the surface, and the direction of integration for the contour integral is found using the right-hand-rule with reference to the surface's normal, as seen in Figure 1 a). For integration on a closed surface, the normal is assumed to point outward from the interior of the surface, and any associated volume integral is with reference to the region enclosed by the surface, see Figure $1 \mathrm{~b}$ ).

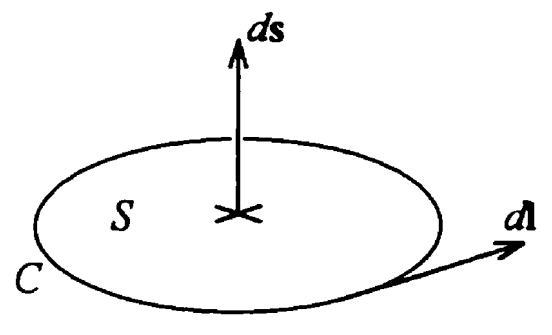

a)

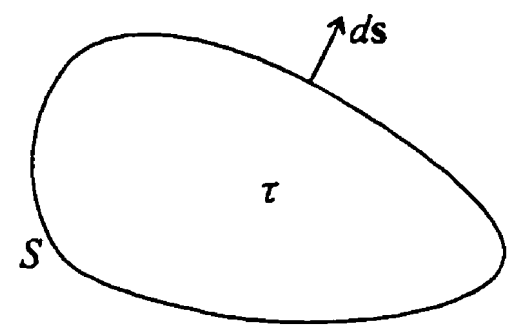

b)

Figure 1: a) $d \mathbf{l}$ and $d s$ on an open surface, b) $d s$ on a closed surface

If the electromagnetic fields are continuous functions with continuous derivatives, the integral equations can be cast in the more familiar differential form of the Maxwell equations

$$
\begin{array}{ll}
\nabla \times \mathbf{E}=-\frac{\partial \mathbf{B}}{\partial t} & \nabla \cdot \mathbf{B}=0 \\
\nabla \times \mathbf{H}=\frac{\partial \mathbf{D}}{\partial t}+\mathbf{J} & \nabla \cdot \mathbf{D}=q_{v}
\end{array}
$$

Contained within this set of equations is the equation of continuity

$$
\nabla \cdot \mathbf{J}=-\frac{\partial q_{v}}{\partial t}
$$

In addition to the above equations, there are several constraints on the fields due to the properties of the materials in which they exist. For a large number of materials the relationships are relatively straightforward and are given by: 


$$
\begin{aligned}
& \mathbf{D}=\boldsymbol{\varepsilon} \mathbf{E} \\
& \mathbf{B}=\boldsymbol{\mu} \mathbf{H} \\
& \mathbf{J}=\sigma \mathbf{E}
\end{aligned}
$$

The above constitutive relations introduce $\varepsilon$, the electrical permittivity of the medium, $\mu$, the magnetic permeability of the medium, and $\sigma$, the medium's conductivity. Materials with a large conductivity are called conductors, and those with a small conductivity are called insulators or dielectrics. To simplify the calculations it is often easier to consider a conductor as being perfect, having an infinite conductivity, and a dielectric as being perfect, having a zero conductivity.

If there are no current sources present, the curl equations fully describe all the relevant physics (see, for example, [72]). Some implications of dropping the divergence relations are discussed later in the thesis. When the differential form of Maxwell's curl equations are written in Cartesian coordinates the following six coupled partial differential equations are obtained:

$$
\begin{gathered}
\frac{\partial B_{x}}{\partial t}=\frac{\partial E_{y}}{\partial z}-\frac{\partial E_{z}}{\partial y} \\
\frac{\partial B_{y}}{\partial t}=\frac{\partial E_{z}}{\partial x}-\frac{\partial E_{x}}{\partial z} \\
\frac{\partial B_{z}}{\partial t}=\frac{\partial E_{x}}{\partial y}-\frac{\partial E_{y}}{\partial x} \\
\frac{\partial D_{x}}{\partial t}=\frac{\partial H_{z}}{\partial y}-\frac{\partial H_{y}}{\partial z}-J_{x} \\
\frac{\partial D_{y}}{\partial t}=\frac{\partial H_{x}}{\partial z}-\frac{\partial H_{z}}{\partial x}-J_{y} \\
\frac{\partial D_{z}}{\partial t}=\frac{\partial H_{y}}{\partial x}-\frac{\partial H_{x}}{\partial y}-J_{z}
\end{gathered}
$$

\subsection{Two-Dimensional Equations}

If all the field values and source terms have no variation in the $z$ direction, the twodimensional form of the equations can be obtained by setting all the $z$ derivatives in ( 2.6 ) to 
zero. When this is done the six partial differential equations (PDEs) from the three-dimensional case decouple into the following two sets of equations:

$$
\begin{gathered}
\frac{\partial \varepsilon E_{z}}{\partial t}=\frac{\partial H_{y}}{\partial x}-\frac{\partial H_{x}}{\partial y}-J_{z} \\
\frac{\partial \mu H_{x}}{\partial t}=-\frac{\partial E_{z}}{\partial y} \\
\frac{\partial \mu H_{y}}{\partial t}=\frac{\partial E_{z}}{\partial x} \\
\frac{\partial \mu H_{z}}{\partial t}=\frac{\partial E_{x}}{\partial y}-\frac{\partial E_{y}}{\partial x} \\
\frac{\partial \varepsilon E_{x}}{\partial t}=\frac{\partial H_{z}}{\partial y}-J_{x} \\
\frac{\partial \varepsilon E_{y}}{\partial t}=-\frac{\partial H_{z}}{\partial x}-J_{y}
\end{gathered}
$$

The constitutive relationships given in ( 2.5 ) have been used to eliminate the electric and magnetic flux densities. Equation ( 2.7 ) contains the unknown field values $E_{z}, H_{x}$, and $H_{y}$, and is known as the Transverse Magnetic (TM) set of equations because the magnetic field vector lies in the $x-y$ plane. The Transverse Electric (TE) set of equations, given by ( 2.8 ), relates the unknown field values $H_{z}, E_{x}$, and $E_{y}$, and has an electric field vector which lies in the $x-y$ plane. A general solution to Maxwell's equations can always be written as the sum of a TM field and a TE field. It should be noted that the TM and TE equations are very similar in form, ignoring sources and boundary conditions, and can be shown to describe the same physics [19].

Consider a linear ( $\varepsilon$ and $\mu$ independent of $|\mathbf{E}|$ and $|\mathbf{H}|$ ), isotropic ( $\varepsilon$ and $\mu$ scalar), perfect dielectric $(\sigma=0)$ material, with no charge density $\left(q_{v}=0\right)$, and no current sources $(\mathbf{J}=\mathbf{0})$. Under these conditions, the TM equations can be expressed in conservative form as:

$$
\frac{\partial \mathbf{Q}}{\partial t}+\frac{\partial \mathbf{F}}{\partial x}+\frac{\partial \mathbf{G}}{\partial y}=\mathbf{0}
$$

where the vector of unknowns is given by:

$$
\mathbf{Q}=\left[\begin{array}{lll}
D: & B_{x} & B_{y}
\end{array}\right]^{T}
$$

and 


$$
\mathbf{F}=\left[\begin{array}{c}
-\frac{1}{\mu} B_{y} \\
0 \\
-\frac{1}{\varepsilon} D_{z}
\end{array}\right] \quad \mathbf{G}=\left[\begin{array}{c}
\frac{1}{\mu} B_{x} \\
\frac{1}{\varepsilon} D_{z} \\
0
\end{array}\right]
$$

Note that $\mathbf{F}=\mathbf{A Q}$ and $\mathbf{G}=\mathbf{B Q}$ where the two matrices are

$$
\mathbf{A}=\left[\begin{array}{ccc}
0 & 0 & -\frac{1}{\mu} \\
0 & 0 & 0 \\
-\frac{1}{\varepsilon} & 0 & 0
\end{array}\right] \quad \mathbf{B}=\left[\begin{array}{ccc}
0 & \frac{1}{\mu} & 0 \\
\frac{1}{\varepsilon} & 0 & 0 \\
0 & 0 & 0
\end{array}\right]
$$

If these matrices have constant coefficients with respect to position, that is, $\varepsilon$ and $\mu$ do not vary spatially in the domain being considered, they can be brought outside of the derivative terms, resulting in the following equation:

$$
\frac{\partial \mathbf{Q}}{\partial t}+\mathbf{A} \frac{\partial \mathbf{Q}}{\partial x}+\mathbf{B} \frac{\partial \mathbf{Q}}{\partial y}=\mathbf{0}
$$

\subsection{2-D Equations in Curvilinear Coordinates}

When numerically modeling electromagnetic fields, it is often the case that the geometry of the problem lends itself to a generalized coordinate system. Anderson, Tannehill, and Pletcher [1] present a method which allows the independent variables of the partial differential equations to be transformed into a uniformly spaced computational domain. The two-dimensional TM form of Maxwell's curl equations can be expressed in a generalized coordinate system using the following transformation:

$$
\xi=\xi(x, y) \quad \eta=\eta(x, y)
$$

Expressing the derivatives in equation ( 2.9 ) in terms of $\xi$ and $\eta$ leads to

$$
\frac{\partial \mathbf{Q}}{\partial t}+\frac{\partial \xi}{\partial x} \frac{\partial \mathbf{F}}{\partial \xi}+\frac{\partial \eta}{\partial x} \frac{\partial \mathbf{F}}{\partial \eta}+\frac{\partial \xi}{\partial y} \frac{\partial \mathbf{G}}{\partial \xi}+\frac{\partial \eta}{\partial y} \frac{\partial \mathbf{G}}{\partial \eta}=\mathbf{0}
$$


The above equation introduces some new terms, $\frac{\partial \xi}{\partial x}, \frac{\partial \eta}{\partial x}, \frac{\partial \xi}{\partial y}$, and $\frac{\partial \eta}{\partial y}$, which are known as the grid metrics because they are completely dependent on the computational grid. Using simple calculus it can be shown that the metrics are given by

$$
\frac{\partial \xi}{\partial x}=J \frac{\partial y}{\partial \eta} \quad \frac{\partial \xi}{\partial y}=-J \frac{\partial x}{\partial \eta} \quad \frac{\partial \eta}{\partial x}=-J \frac{\partial y}{\partial \xi} \quad \frac{\partial \eta}{\partial y}=J \frac{\partial x}{\partial \xi}
$$

where the Jacobian of the transformation can be determined from the following

$$
J^{-1}=\frac{\partial x}{\partial \xi} \frac{\partial y}{\partial \eta}-\frac{\partial x}{\partial \eta} \frac{\partial y}{\partial \xi}
$$

The right hand side terms in ( 2.16 ) can be calculated numerically as shown in $\S 3.1 .1$.

Equation (2.15) is often referred to as the chain rule form of Maxwell's equations in generalized coordinates. To simplify the determination of the characteristic variables for this equation, which is illustrated in the next section, it is advantageous to have the grid metrics inside the spatial derivatives, obtaining the strong conservation law form. The strong conservation law form is essential if the material properties are not constant. This can be done by multiplying equation ( 2.15 ) by the inverse of the Jacobian, and after manipulating the resulting equations using some simple calculus (see Appendix A), the following result is obtained

$$
\frac{\partial \hat{\mathbf{Q}}}{\partial t}+\frac{\partial \hat{\mathbf{F}}}{\partial \xi}+\frac{\partial \hat{\mathbf{G}}}{\partial \eta}=\mathbf{0}
$$

where $\hat{\mathbf{F}}=\hat{\mathbf{A}} \hat{\mathbf{Q}}$ and $\hat{\mathbf{G}}=\hat{\mathbf{B}} \hat{\mathbf{Q}}$. The vector of unknowns is given by:

$$
\hat{\mathbf{Q}}=\frac{1}{J}\left[\begin{array}{lll}
D_{z} & B_{x} & B_{y}
\end{array}\right]^{\tau}
$$

and the two matrices are

$$
\hat{\mathbf{A}}=\left[\begin{array}{ccc}
0 & \frac{1}{\mu} \frac{\partial \xi}{\partial y} & -\frac{1}{\mu} \frac{\partial \xi}{\partial x} \\
\frac{1}{\varepsilon} \frac{\partial \xi}{\partial y} & 0 & 0 \\
-\frac{1}{\varepsilon} \frac{\partial \xi}{\partial x} & 0 & 0
\end{array}\right] \quad \hat{\mathbf{B}}=\left[\begin{array}{ccc}
0 & \frac{1}{\mu} \frac{\partial \eta}{\partial y} & -\frac{1}{\mu} \frac{\partial \eta}{\partial x} \\
\frac{1}{\varepsilon} \frac{\partial \eta}{\partial y} & 0 & 0 \\
-\frac{1}{\varepsilon} \frac{\partial \eta}{\partial x} & 0 & 0
\end{array}\right]
$$

The above matrices are related by the following simple transformation

$$
\hat{\mathbf{A}} \underset{\xi \leftrightarrow \eta}{\longrightarrow} \hat{\mathbf{B}}
$$


That is, one can obtain $\hat{\mathbf{B}}$ from $\hat{\mathbf{A}}$ by replacing all occurrences of $\xi$ with $\eta$.

When the material properties of the domain do not vary spatially, the generalized coordinate transformation can be applied to ( 2.13 ). This results in the following equation:

$$
\frac{\partial \mathbf{Q}}{\partial t}+\mathbf{A}\left(\frac{\partial \xi}{\partial x} \frac{\partial \mathbf{Q}}{\partial \xi}+\frac{\partial \eta}{\partial x} \frac{\partial \mathbf{Q}}{\partial \eta}\right)+\mathbf{B}\left(\frac{\partial \xi}{\partial y} \frac{\partial \mathbf{Q}}{\partial \xi}+\frac{\partial \eta}{\partial y} \frac{\partial \mathbf{Q}}{\partial \eta}\right)=\mathbf{0}
$$

After grouping on the derivatives of $\mathbf{Q}$, the above equation can be written as

$$
\frac{\partial \mathbf{Q}}{\partial t}+\hat{\mathbf{A}} \frac{\partial \mathbf{Q}}{\partial \xi}+\hat{\mathbf{B}} \frac{\partial \mathbf{Q}}{\partial \eta}=\mathbf{0}
$$

where $\hat{\mathbf{A}}$ and $\hat{\mathbf{B}}$ are given above.

\subsection{Characteristic Variables}

It is often invaluable to have some knowledge about how disturbances propagate along the spatial coordinate directions. This proves to be especially useful when trying to determine what types of disturbances are moving towards and away from a boundary, whether it be an interface between different types of materials or the outer boundary of a computational domain. This can be accomplished using the method of characteristics as outlined, for example, by Shang [56]. By applying this method to the two-dimensional TM equations, one can determine the characteristic variables which propagate in the positive and negative directions of both of the spatial coordinates.

\subsubsection{Characteristic Variables in Cartesian Coordinates}

The matrix $\mathbf{A}$ can be diagonalized using the following transformation:

$$
\mathrm{X}_{1}^{-1} \mathrm{AX} \mathrm{X}_{1}=\Lambda_{1}
$$

The matrices in the transformation can be found by determining the eigenvalues of $A$ and placing them along the diagonal of $\Lambda_{A}$, and finding the right eigenvectors of $A$ and placing them in the columns of $X_{A}$. This gives:

$$
\Lambda_{A}=\left[\begin{array}{ccc}
0 & 0 & 0 \\
0 & c & 0 \\
0 & 0 & -c
\end{array}\right]
$$




$$
\mathbf{X}_{A}=\left[\begin{array}{ccc}
0 & -1 & 1 \\
1 & 0 & 0 \\
0 & \eta_{1} & \eta_{I}
\end{array}\right] \quad \mathbf{X}_{A}^{-1}=\left[\begin{array}{ccc}
0 & 1 & 0 \\
-\frac{1}{2} & 0 & \frac{1}{2 \eta_{I}} \\
\frac{1}{2} & 0 & \frac{1}{2 \eta_{I}}
\end{array}\right]
$$

where $c=\frac{1}{\sqrt{\varepsilon \mu}}$ is the speed of light in the medium being considered, and $\eta_{I}=\sqrt{\frac{\mu}{\varepsilon}}$ is the intrinsic impedance of the medium.

In a similar fashion, the matrix $\mathbf{B}$ can be diagonalized as follows:

$$
\mathrm{X}_{B}^{-1} \mathrm{BX}_{B}=\Lambda_{B}
$$

where

$$
\begin{gathered}
\Lambda_{B}=\left[\begin{array}{ccc}
0 & 0 & 0 \\
0 & c & 0 \\
0 & 0 & -c
\end{array}\right] \\
\mathbf{X}_{B}=\left[\begin{array}{ccc}
0 & 1 & -1 \\
0 & \eta_{I} & \eta_{I} \\
1 & 0 & 0
\end{array}\right] \quad \mathbf{X}_{B}^{-1}=\left[\begin{array}{ccc}
0 & 0 & 1 \\
\frac{1}{2} & \frac{1}{2 \eta_{I}} & 0 \\
-\frac{1}{2} & \frac{1}{2 \eta_{I}} & 0
\end{array}\right]
\end{gathered}
$$

A single diagonal matrix, $\Lambda=\Lambda_{A}=\Lambda_{B}$, can be defined.

One can split the diagonal matrix into two parts, one containing positive eigenvalues and the other negative eigenvalues, as follows:

$$
\Lambda^{ \pm}=\frac{\Lambda \pm|\Lambda|}{2}
$$

This results in the following two matrices:

$$
\Lambda^{+}=\left[\begin{array}{ccc}
0 & 0 & 0 \\
0 & c & 0 \\
0 & 0 & 0
\end{array}\right] \quad \Lambda^{-}=\left[\begin{array}{ccc}
0 & 0 & 0 \\
0 & 0 & 0 \\
0 & 0 & -c
\end{array}\right]
$$

These matrices can be used to define the following:

$$
A^{ \pm}=X_{A} \Lambda^{ \pm} X_{A}^{-1}
$$




$$
\mathbf{B}^{ \pm}=\mathbf{X}_{B} \Lambda^{ \pm} \mathbf{X}_{B}^{-1}
$$

These equations lead to:

$$
\begin{aligned}
& \mathbf{A}^{ \pm}=\frac{1}{2}\left[\begin{array}{ccc} 
\pm c & 0 & -\frac{1}{\mu} \\
0 & 0 & 0 \\
-\frac{1}{\varepsilon} & 0 & \pm c
\end{array}\right] \\
& \mathbf{B}^{ \pm}=\frac{1}{2}\left[\begin{array}{ccc} 
\pm c & \frac{1}{\mu} & 0 \\
\frac{1}{\varepsilon} & \pm c & 0 \\
0 & 0 & 0
\end{array}\right]
\end{aligned}
$$

The above matrices can be used to define the following:

$$
\begin{aligned}
& F^{ \pm}=\mathbf{A}^{ \pm} \mathbf{Q} \\
& \mathbf{G}^{ \pm}=\mathbf{B}^{ \pm} \mathbf{Q}
\end{aligned}
$$

The resulting vectors are:

$$
\begin{aligned}
& \mathbf{F}^{ \pm}=\frac{1}{2}\left[\begin{array}{c} 
\pm c D_{z}-\frac{1}{\mu} B_{y} \\
0 \\
-\frac{1}{\varepsilon} D_{z} \pm c B_{y}
\end{array}\right]=\frac{1}{2}\left[\begin{array}{c} 
\pm \frac{1}{\mu} \\
0 \\
-c
\end{array}\right]\left(\eta_{1} D_{z} \mp B_{y}\right) \\
& \mathbf{G}^{ \pm}=\frac{1}{2}\left[\begin{array}{c} 
\pm c D_{z}+\frac{1}{\mu} B_{x} \\
\frac{1}{\varepsilon} D_{z} \pm c B_{x} \\
0
\end{array}\right]=\frac{1}{2}\left[\begin{array}{c}
\frac{1}{\mu} \\
c \\
0
\end{array}\right]\left(\eta_{1} D_{z} \pm B_{x}\right)
\end{aligned}
$$

Note that $\mathbf{F}=\mathbf{F}^{+}+\mathbf{F}^{-}$and $\mathbf{G}=\mathbf{G}^{+}+\mathbf{G}^{-}$. The above equations show that the characteristic variables which propagate in the positive and negative $x$ directions are $\eta_{t} D_{z}-B_{y}$ and $\eta_{s} D_{z}+B_{y}$, respectively, and the characteristic variables which propagate in the positive and negative $y$ directions are $\eta_{t} D_{z}+B_{x}$ and $\eta_{1} D_{z}-B_{x}$, respectively. 


\subsubsection{Characteristic Variables in Generalized Coordinates}

The $\hat{\mathbf{A}}$ matrix can be diagonalized using the following transformation:

$$
\mathrm{X}_{i}^{-1} \hat{\mathrm{A}} \mathrm{X}_{\boldsymbol{i}}=\Lambda_{\hat{i}}
$$

where

$$
\begin{gathered}
\Lambda_{i}=\left[\begin{array}{ccc}
0 & 0 & 0 \\
0 & c \mid \nabla \xi & 0 \\
0 & 0 & -c|\nabla \xi|
\end{array}\right] \\
\mathbf{X}_{i}=\left[\begin{array}{ccc}
0 & -|\nabla \xi| & |\nabla \xi| \\
\frac{\partial \xi}{\partial x} & -\eta_{t} \frac{\partial \xi}{\partial y} & -\eta_{1} \frac{\partial \xi}{\partial y} \\
\frac{\partial \xi}{\partial y} & \eta_{t} \frac{\partial \xi}{\partial x} & \eta_{t} \frac{\partial \xi}{\partial x}
\end{array}\right] \quad \mathbf{X}_{\dot{i}}^{-1}=\left[\begin{array}{ccc}
0 & \frac{1}{|\nabla \xi|^{2}} \frac{\partial \xi}{\partial x} & \frac{1}{|\nabla \xi|^{2}} \frac{\partial \xi}{\partial y} \\
-\frac{1}{2|\nabla \xi|} & -\frac{1}{2 \eta_{l}|\nabla \xi|^{2}} \frac{\partial \xi}{\partial y} & \frac{1}{2 \eta_{I}|\nabla \xi|^{2}} \frac{\partial \xi}{\partial x} \\
\frac{1}{2|\nabla \xi|} & -\frac{1}{2 \eta_{l}|\nabla \xi|^{2}} \frac{\partial \xi}{\partial y} & \frac{1}{2 \eta_{l}|\nabla \xi|^{2}} \frac{\partial \xi}{\partial x}
\end{array}\right] \\
\text { and } \nabla \xi=\left(\frac{\partial \xi}{\partial x}, \frac{\partial \xi}{\partial y}\right) \text { has a magnitude given by }|\nabla \xi|=\sqrt{\left(\frac{\partial \xi}{\partial x}\right)^{2}+\left(\frac{\partial \xi}{\partial y}\right)^{2}} .
\end{gathered}
$$

Splitting the diagonal matrix results in the following two matrices:

$$
\Lambda_{i}^{+}=\left[\begin{array}{ccc}
0 & 0 & 0 \\
0 & c|\nabla \xi| & 0 \\
0 & 0 & 0
\end{array}\right] \quad \Lambda_{i}^{-}=\left[\begin{array}{ccc}
0 & 0 & 0 \\
0 & 0 & 0 \\
0 & 0 & -c|\nabla \xi|
\end{array}\right]
$$

These matrices can be used to define the following:

$$
\hat{\mathbf{A}}^{ \pm}=\mathbf{X}_{\hat{i}} \Lambda_{\dot{i}}^{ \pm} \mathbf{X}_{\hat{i}}^{-1}
$$

This equation leads to:

$$
\hat{\mathbf{A}}^{ \pm}=\frac{1}{2}\left[\begin{array}{ccc} 
\pm c|\nabla \xi| & \frac{1}{\mu} \frac{\partial \xi}{\partial y} & -\frac{1}{\mu} \frac{\partial \xi}{\partial x} \\
\frac{1}{\varepsilon} \frac{\partial \xi}{\partial y} & \pm c \frac{1}{|\nabla \xi|}\left(\frac{\partial \xi}{\partial y}\right)^{2} & \mp c \frac{1}{|\nabla \xi|} \frac{\partial \xi}{\partial x} \frac{\partial \xi}{\partial y} \\
-\frac{1}{\varepsilon} \frac{\partial \xi}{\partial x} & \mp c \frac{1}{|\nabla \xi|} \frac{\partial \xi}{\partial x} \frac{\partial \xi}{\partial y} & \pm c \frac{1}{|\nabla \xi|}\left(\frac{\partial \xi}{\partial x}\right)^{2}
\end{array}\right]
$$

The above matrix can be used to define:

$$
\hat{\mathbf{F}}^{ \pm}=\hat{\mathbf{A}}^{ \pm} \hat{\mathbf{Q}}
$$


The resulting vectors are:

$$
\hat{\mathbf{F}}^{ \pm}=\frac{1}{2 J}\left[\begin{array}{c} 
\pm c|\nabla \xi| D_{z}+\frac{1}{\mu}\left(\frac{\partial \xi}{\partial y} B_{x}-\frac{\partial \xi}{\partial x} B_{y}\right) \\
\frac{\partial \xi}{\partial y}\left(\frac{1}{\varepsilon} D_{z} \pm c \frac{\left(\frac{\partial \xi}{\partial y} B_{x}-\frac{\partial \xi}{\partial x} B_{y}\right)}{|\nabla \xi|}\right) \\
-\frac{\partial \xi}{\partial x}\left(\frac{1}{\varepsilon} D_{z} \pm c \frac{\left(\frac{\partial \xi}{\partial y} B_{x}-\frac{\partial \xi}{\partial x} B_{y}\right)}{|\nabla \xi|}\right)
\end{array}\right]=\frac{1}{2 J}\left[\begin{array}{c} 
\pm \frac{|\nabla \xi|}{\mu} \\
c \frac{\partial \xi}{\partial y} \\
-c \frac{\partial \xi}{\partial x}
\end{array}\right]\left(\eta_{t} D_{z} \pm \frac{\left(\frac{\partial \xi}{\partial y} B_{x}-\frac{\partial \xi}{\partial x} B_{y}\right)}{|\nabla \xi|}\right)
$$

Using (2.21) we can easily see that

$$
\hat{\mathbf{G}}^{ \pm}=\frac{1}{2 J}\left[\begin{array}{c} 
\pm c|\nabla \eta| D_{z}+\frac{1}{\mu}\left(\frac{\partial \eta}{\partial y} B_{x}-\frac{\partial \eta}{\partial x} B_{y}\right) \\
\frac{\partial \eta}{\partial y}\left(\begin{array}{c}
\left.\frac{1}{\varepsilon} D_{z} \pm c \frac{\left(\frac{\partial \eta}{\partial y} B_{x}-\frac{\partial \eta}{\partial x} B_{y}\right)}{|\nabla \eta|}\right) \\
-\frac{\partial \eta}{\partial x}\left(\frac{1}{\varepsilon} D_{z} \pm c \frac{\left(\frac{\partial \eta}{\partial y} B_{x}-\frac{\partial \eta}{\partial x} B_{y}\right)}{|\nabla \eta|}\right)
\end{array}\right]=\frac{1}{2 J}\left[\begin{array}{c} 
\pm \frac{|\nabla \eta|}{\mu} \\
c \frac{\partial \eta}{\partial y} \\
-c \frac{\partial \eta}{\partial x}
\end{array}\right]\left(\eta_{t} D_{z} \pm \frac{\left(\frac{\partial \eta}{\partial y} B_{x}-\frac{\partial \eta}{\partial x} B_{y}\right)}{|\nabla \eta|}\right)
\end{array}\right.
$$

Note that $\hat{\mathbf{F}}=\hat{\mathbf{F}}^{+}+\hat{\mathbf{F}}^{-}$and $\hat{\mathbf{G}}=\hat{\mathbf{G}}^{+}+\hat{\mathbf{G}}^{-}$. The above equations show that the characteristic variables which propagate in the positive and negative $\xi$ directions are $\eta_{1} D_{z}+\left(\frac{\partial \xi}{\partial y} B_{x}-\frac{\partial \xi}{\partial x} B_{y}\right) /|\nabla \xi|$ and $\eta_{1} D_{z}-\left(\frac{\partial \xi}{\partial y} B_{x}-\frac{\partial \xi}{\partial x} B_{y}\right) /|\nabla \xi|$, respectively, and the 
characteristic variables which propagate in the positive and negative $\eta$ directions are $\eta_{1} D_{z}+\left(\frac{\partial \eta}{\partial y} B_{x}-\frac{\partial \eta}{\partial x} B_{y}\right) /|\nabla \eta|$ and $\eta_{1} D_{z}-\left(\frac{\partial \eta}{\partial y} B_{x}-\frac{\partial \eta}{\partial x} B_{y}\right) /|\nabla \eta|$, respectively.

\subsection{Boundary Conditions}

Only the simplest problems in electromagnetics do not involve any boundary conditions. One such problem is the propagation of electromagnetic waves in free space, for which analytical solutions exist. In general, problems which introduce objects composed of materials having different types of electromagnetic properties are much more challenging. To obtain solutions for these types of problems, the boundary conditions at the interfaces between the objects must be determined. The following boundary conditions can be found in many texts, such as Umashankar's and Taflove's [78], which illustrates their development quite clearly.

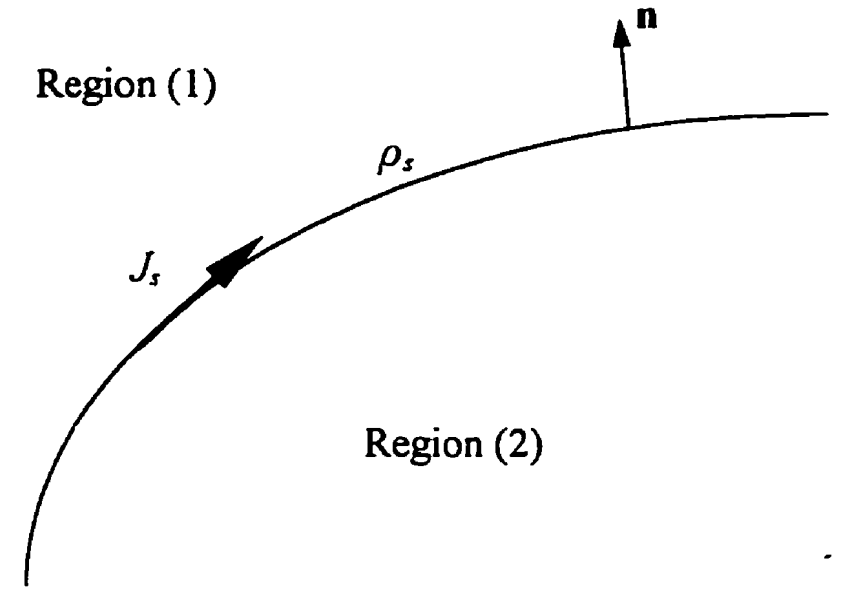

Figure 2: Surface currents and charge density on material interface

Consider the general case of any two materials as shown in Figure 2. On the material interface the following conditions must hold:

$$
\begin{gathered}
E_{t}^{(1)}=E_{t}^{(2)} \\
H_{t}^{(1)}-H_{t}^{(2)}=J_{s} \\
D_{n}^{(1)}-D_{n}^{(2)}=\rho_{s} \\
B_{n}^{(1)}=B_{n}^{(2)}
\end{gathered}
$$


The above four equations state that the tangential electric field intensity and normal magnetic flux density must be continuous across the interface, the difference in the tangential magnetic intensities at the interface is equal to the surface current flowing along the interface, and the discontinuity in the normal electric flux density is equal to the surface charge density on the interface. These relations include the effects of a surface current and a surface charge density. For many problems, these values will be identically zero.

If Region (2) is a perfect conductor, that is, having an infinite conductivity, then all the field values inside the region must be zero. Using this fact, and the above four relations, the boundary conditions at the surface of a perfect conductor are obtained

$$
\begin{aligned}
& E_{t}^{(1)}=0 \\
& H_{t}^{(1)}=J_{s} \\
& D_{n}^{(1)}=\rho_{s} \\
& B_{n}^{(1)}=0
\end{aligned}
$$

In general there will always be a finite current density on the surface of a perfect conductor, but there does not have to be a surface charge density for the TM case in two dimensions. 


\section{Numerical Methods}

This research is concerned with simulating the propagation and scattering of electromagnetic waves. The scatterers are made up of linear isotropic perfect dielectrics, perfect conductors, or combinations of the two. A separate numerical domain is used for each material, and the proper physical conditions are implemented along interfaces to allow the transmission and reflection of wave fronts. To solve for the total electric and magnetic field components in each numerical domain, Maxwell's equations are approximated using finite-difference methods.

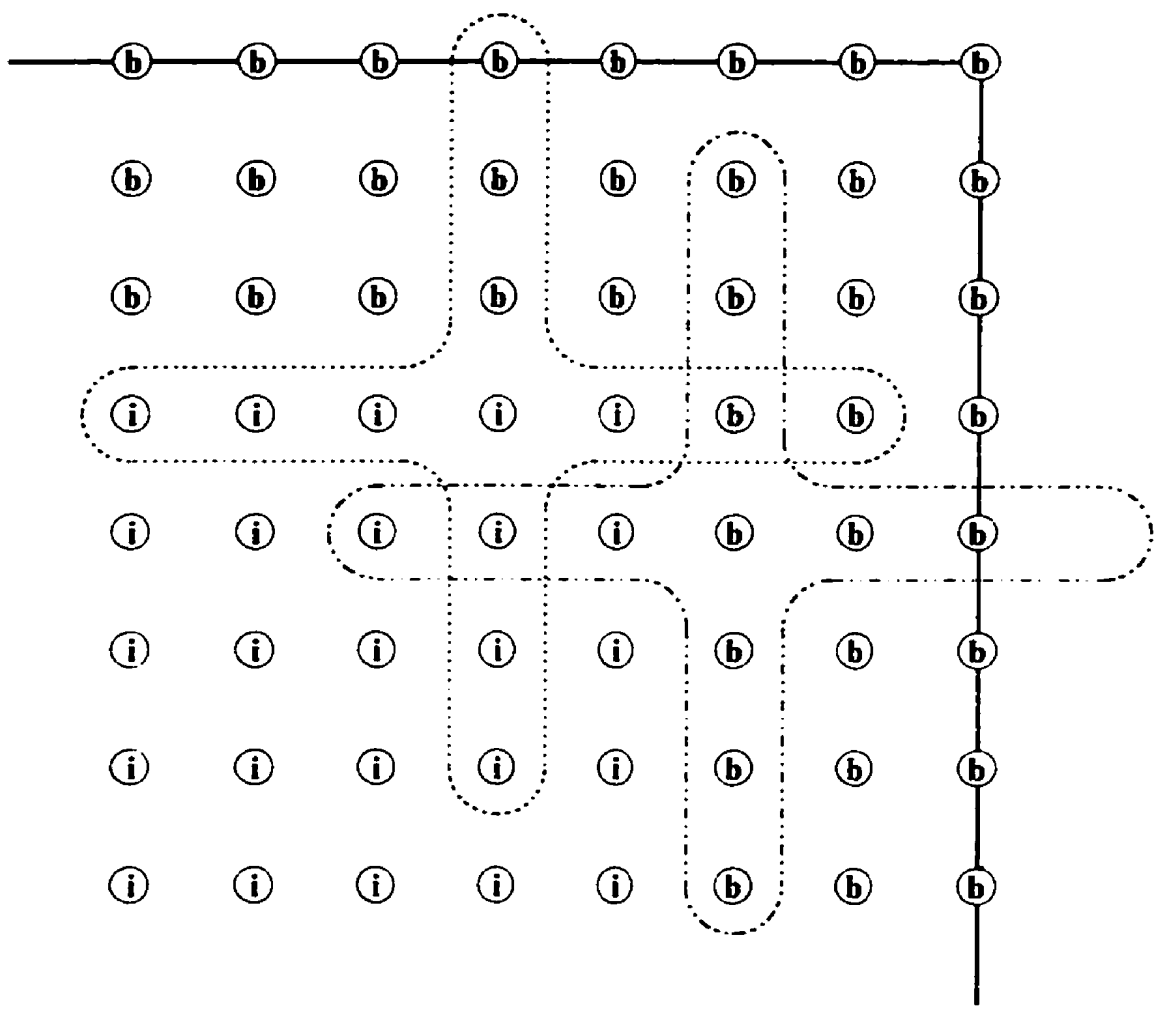

Figure 3: Interior, $\mathbf{i}$, and boundary, $b$, nodes near corner of domain for a seven point cross computational stencil

Any grid which is used to discretize a numerical domain will contain nodes which are part of the interior region and nodes which are part of the boundary region, as shown in Figure 3. The computational stencil of the spatial operator which is used in the interior of the domain defines which region each node falls in. When the interior finite-difference scheme is applied at a node, if any part of the stencil falls outside the domain's boundary the node is considered to be in the boundary region, otherwise it is considered to be in the interior region. 
This chapter describes both the finite-difference operators that are used in the interior of the numerical domain, and the operators which are implemented near a boundary, referred to as a numerical boundary scheme.

\subsection{Interior Finite-Difference Schemes}

To approximate the governing equations, the finite-difference methods are applied in two parts: a spatial-operator is used to obtain the spatial derivatives of the field values, and a time-marching method is used to calculate the field values at a later time, using some initial conditions. The methods described below were originally presented by Zingg et al. [89], and an extensive analysis of the methods can be found in [95].

The spatial operator is made up of two parts, an antisymmetric or central-difference operator:

$$
\left(\delta_{x}^{a} u\right)_{i}=\frac{1}{\Delta x}\left[a_{1}\left(u_{i+1}-u_{i-1}\right)+a_{2}\left(u_{i+2}-u_{i-2}\right)+a_{3}\left(u_{i+3}-u_{i-3}\right)\right]
$$

and a symmetric operator:

$$
\left(\delta_{x}^{s} u\right)_{i}=\frac{1}{\Delta x}\left[d_{0} u_{1}+d_{1}\left(u_{i+1}+u_{i-1}\right)+d_{2}\left(u_{i+2}+u_{i-2}\right)+d_{3}\left(u_{i+3}+u_{1-3}\right)\right]
$$

The family of operators described by (3.1) has a maximum seven-point stencil, and the operator with the maximum order of accuracy has a truncation error which varies as $\Delta x^{6}$. The family of operators described by ( 3.2 ) also has a maximum seven-point stencil, but its maximum-order operator has a truncation error which varies as $\Delta x^{5}$. The symmetric operator is used to add a small amount of artificial dissipation to the scheme. When the operator is applied to a hyperbolic system of equations, a characteristic splitting is used. For example, if the equation being modeled contains the term $\frac{\partial \mathbf{A u}}{\partial x}$, it is approximated by

$$
\left(\frac{\partial \mathbf{A u}}{\partial x}\right)_{i}=\left(\delta_{x}^{a} \mathbf{A u}\right)_{i}+\left(\delta_{x}^{s}|\mathbf{A}| \mathbf{u}\right)_{i}
$$

at any interior node $i$. Note that $|A|=X|\Lambda| X^{-1}$ where $X$ is the matrix of right eigenvectors of $A$, and $\Lambda$ is the matrix of eigenvalues of $\mathbf{A}$. If the matrix $\mathbf{A}$ can be brought outside the derivative the following approximation is used 


$$
\mathbf{A}\left(\frac{\partial \mathbf{u}}{\partial x}\right)_{i}=\mathbf{A}\left(\delta_{x}^{a} \mathbf{u}\right)_{i}+|\mathbf{A}|\left(\delta_{x}^{s} \mathbf{u}\right)_{i}
$$

The method is applied in a similar way to approximate a $y$ derivative. For the present set of equations one needs

$$
|\hat{\mathbf{A}}|=\left[\begin{array}{ccc}
c|\nabla \xi| & 0 & 0 \\
0 & c \frac{1}{|\nabla \xi|}\left(\frac{\partial \xi}{\partial y}\right)^{2} & -c \frac{1}{|\nabla \xi|} \frac{\partial \xi}{\partial x} \frac{\partial \xi}{\partial y} \\
0 & -c \frac{1}{|\nabla \xi|} \frac{\partial \xi}{\partial x} \frac{\partial \xi}{\partial y} & c \frac{1}{|\nabla \xi|}\left(\frac{\partial \xi}{\partial x}\right)^{2}
\end{array}\right]
$$

and $|\hat{B}|$ which can easily be obtained using ( 2.21 ) and the above relation.

The two spatial operators given above can be used to represent many different finitedifference schemes. Taylor series expansions can be used to determine the schemes' truncation errors [1]. The following constraint on the coefficients of (3.1) is needed to obtain a secondorder method

$$
a_{1}+2 a_{2}+3 a_{3}=1 / 2
$$

A fourth-order method is obtained by imposing the additional constraint

$$
a_{1}+2^{3} a_{2}+3^{3} a_{3}=0
$$

and the method with the highest possible accuracy, sixth-order, is obtained by adding

$$
a_{1}+2^{5} a_{2}+3^{5} a_{3}=0
$$

The following constraint on the coefficients of (3.2) is needed to obtain a first-order method

$$
\frac{1}{2} d_{0}+d_{1}+d_{2}+d_{3}=0
$$

A third-order method is obtained by imposing the additional constraint

$$
d_{1}+2^{2} d_{2}+3^{2} d_{3}=0
$$

and the method with the highest possible accuracy, fifth-order, is obtained by adding

$$
d_{1}+2^{4} d_{2}+3^{4} d_{3}=0
$$

Using the above constraints for the two spatial operators, a number of different families of schemes can be obtained. These families are defined by the number of parameters available, or free coefficients, and the order of the approximation. Table 1 gives the coefficients and 
truncation error for a two-parameter family of second-order central-difference schemes, a one-parameter family of fourth-order central-difference schemes, and the sixth-order centraldifference scheme. Setting $a_{2}=a_{3}=0$ in the two-parameter family gives the classical secondorder central-difference scheme, and setting $a_{3}=0$ in the one-parameter family gives the classical fourth-order central-difference scheme.

\begin{tabular}{|c|c|c|c|c|}
\hline Family & $a_{1}$ & $a_{2}$ & $a_{3}$ & Truncation Enor \\
\hline 2 parameter & $1 / 2-2 a_{2}-3 a_{3}$ & - & - & $\left(1 / 2+6 a_{2}+24 a_{3}\right) \Delta x^{2} \frac{d^{3} u}{d x^{3}}+\ldots$ \\
\hline 1 parameter & $1 / 3+5 a_{3}$ & $-\left(1 / 12+4 a_{3}\right)$ & - & $\left(120 a_{3}-2\right) \Delta x^{4} \frac{d^{3} u}{\alpha^{3}}+\ldots$ \\
\hline 0 parameter & $1 / 4$ & $-1 / 20$ & $1 / 60$ & $18 \Delta x^{6} \frac{d^{7} u}{d x^{7}}+\ldots$ \\
\hline
\end{tabular}

Table 1: Coefficients for families of central-difference operators given by equation ( 3.1 )

Table 2 gives the coefficients and truncation error for a three-parameter family of first-order filters, a two-parameter family of third-order filters, and the fifth-order filter with strength $d_{0}$. For the two- and three-parameter families, we generally choose $d_{0}$ to set the strength of the filter and determine the remaining parameters either to optimize the filter (see § 3.1.3) or to reduce the stencil size.

\begin{tabular}{|c|c|c|c|c|}
\hline Family & $d_{1}$ & $d_{2}$ & $d_{3}$ & Truncation Error \\
\hline 3 parameter & $-\left(\frac{1}{2} d_{0}+d_{2}+d_{3}\right)$ & - & - & $\left(-\frac{1}{2} d_{0}+3 d_{2}+8 d_{3}\right) \Delta x \frac{d^{2} u}{\alpha^{2}}+\ldots$ \\
\hline 2 parameter & $-\frac{2}{3} d_{0}+\frac{3}{3} d_{3}$ & $\frac{1}{6} d_{0}-\frac{8}{3} d_{3}$ & - & $\left(2 d_{0}+40 d_{3}\right) \Delta x^{3} \frac{d^{4} u}{\alpha^{4}}+\ldots$ \\
\hline 1 parameter & $-\frac{3}{4} d_{0}$ & $\frac{3}{10} d_{0}$ & $-\frac{1}{20} d_{0}$ & $-18 d_{0} \Delta x^{5} \frac{d^{6} u}{\alpha^{6}}+\ldots$ \\
\hline
\end{tabular}

Table 2: Coefficients for families of filter operators given by equation ( 3.2 )

The time-marching scheme is an explicit six-stage method which, when applied to the following ordinary differential equation (ODE) $\frac{d u}{d t}=f(u, t)$, can be written as: 


$$
\begin{aligned}
& u_{n+\alpha_{1}}^{(1)}=u_{n}+h \alpha_{1} f_{n} \\
& u_{n+\alpha_{2}}^{(2)}=u_{n}+h \alpha_{2} f_{n+\alpha_{1}}^{(1)} \\
& u_{n+a_{3}}^{(3)}=u_{n}+h \alpha_{3} f_{n+\alpha_{2}}^{(2)} \\
& u_{n+\alpha_{4}}^{(4)}=u_{n}+h \alpha_{4} f_{n+a_{3}}^{(3)} \\
& u_{n+\alpha_{3}}^{(5)}=u_{n}+h \alpha_{5} f_{n+a_{4}}^{(4)} \\
& u_{n+1}=u_{n}+h f_{n+\alpha_{3}}^{(5)}
\end{aligned}
$$

Note that $h=\Delta t$ is the time step, $t_{n}=n h, u_{n}=u\left(t_{n}\right)$, and $f_{n+a}^{(k)}=f\left(u_{n+a}^{(k)}, t_{n}+\alpha h\right)$. Setting $\alpha_{5}=1 / 2$ insures that the method will be second-order accurate. Additionally setting $\alpha_{4}=1 / 3$, $\alpha_{3}=1 / 4, \alpha_{2}=1 / 5$, and $\alpha_{1}=1 / 6$ gives the member of this family of methods with the highest formal order for linear homogeneous ODEs. It is sixth-order accurate for such ODEs and second-order accurate for nonlinear or inhomogeneous ODEs. This method generally gives better results than the classical fourth-order Runge-Kutta method when used in conjunction with the above spatial operators to model wave propagation problems. It requires two memory locations per dependent variable, where the classic fourth-order method needs three.

\subsubsection{Approximation of Maxwell's Equations}

To use the time-marching method given by (3.12) to model the 2-D TM set of Maxwell's equations in generalized coordinates, approximations for the time rate of change of the field values must be obtained. These can be found by using the spatial operators ( 3.1 ) and (3.2). When the equations are written in strong conservation law form, (2.18), the following approximation is obtained

$$
\frac{\partial \hat{\mathbf{Q}}}{\partial t} \approx-\left(\delta_{\xi}^{a} \hat{\mathbf{F}}+\delta_{\xi}^{s}|\hat{\mathbf{F}}|\right)-\left(\delta_{\eta}^{a} \hat{\mathbf{G}}+\delta_{\eta}^{s} \mid \hat{\mathbf{G}}\right)
$$

When the material properties of the domain are constant, equation ( 2.23 ) can be used to generate another approximation

$$
\frac{\partial \mathbf{Q}}{\partial t} \approx-\left(\hat{\mathbf{A}} \delta_{\xi}^{a} \mathbf{Q}+|\hat{\mathbf{A}}| \delta_{\xi}^{s} \mathbf{Q}\right)-\left(\hat{\mathbf{B}} \delta_{\eta}^{a} \mathbf{Q}+|\hat{\mathbf{B}}| \delta_{\eta}^{s} \mathbf{Q}\right)
$$

Similar relations for the Cartesian coordinate case can easily be obtained. 
It is important to note that the numerical method used here is not like the staggered uncolocated methods, in that it does not guarantee that the divergence of the magnetic flux density will be zero. Tests have shown that the numerical error in the divergence is on the order of the method's truncation error and is therefore bounded when the initial conditions and incident fields are consistent with the divergence relations.

When using generalized coordinates, the grid metrics must also be numerically approximated. This can be done by using equations (2.16) and (2.17), the $x$ and $y$ locations of the nodes in the grid, and finite-differences to calculate the derivatives of $x$ and $y$ with respect to $\xi$ and $\eta$. To simplify the calculations, $\xi$ and $\eta$ are defined so that the nodes in the grid have integral numbering, i.e. $\xi=i, \eta=j$, and $\Delta \xi=\Delta \eta=1$. The derivatives are approximated using the central-difference operator given by (3.1). For example, the derivative of $x$ with respect to $\xi$ is approximated as follows

$$
\frac{\partial x}{\partial \xi} \approx \delta_{\xi}^{a} x
$$

When using the finite-difference schemes to approximate the strong conservation law form of the equations, care must be taken in order to avoid generating artificial source terms. The existence of these source terms is discussed in Appendix B, and a method for eliminating them is presented. When the coefficient matrices do not vary spatially, and they are brought outside the spatial derivatives, artificial source terms will not be generated. For this reason, whenever possible, Maxwell's equations are simulated using the approximation given by equation ( 3.14 ).

\subsubsection{Fourier Error Analysis}

A Fourier error analysis can be used to determine the errors of the finite-difference methods discussed above; see, for example, [95]. Since the numerical methods are used to solve wave propagation problems, the analysis can be simplified by using a model equation which captures all of the relevant physics. Consider the one-dimensional linear wave equation

$$
\frac{\partial U}{\partial t}+c \frac{\partial U}{\partial x}=0
$$

This equation propagates disturbances in the field variable, $U$, in the positive $x$ direction with a phase and group velocity of $c$. Assume the following solution to this equation 


$$
U(x, t)=u(t) e^{j \times x}
$$

where $j=\sqrt{-1}$ and $\kappa$ is the spatial wavenumber. Substituting this into (3.16) results in the following ODE for the time component of the solution

$$
\frac{d u}{d t}=-j c \kappa u
$$

The exact solution to this equation is simply

$$
u(t)=u_{0} e^{-j \alpha x t}
$$

which gives an analytical solution for ( 3.16$)$ which can be written as

$$
U(x, t)=u_{0} e^{j(x-\omega t)}
$$

where

$$
\omega=c \kappa
$$

is the angular frequency of the wave.

If (3.16) is modeled using a semi-discrete approach, the spatial derivative can be replaced by the finite difference operators given in (3.1) and (3.2). This results in the following numerical equation for the value of $U$ at the node $i$

$$
\left(\frac{\partial U}{\partial t}\right)_{i}+c\left[\left(\delta_{x}^{a} U\right)_{i}+\left(\delta_{x}^{s} U\right)_{i}\right]=0
$$

Assuming a uniform spatial discretization, such that $x_{i}=i \Delta x,(3.17)$ can be used to obtain $U_{i}=u(t) e^{j a \Delta x}$. Substituting this into equation ( 3.22 ) gives

$$
\frac{d u}{d t} e^{j \kappa i \Delta x}+\frac{c}{\Delta x}\left[\begin{array}{c}
a_{1}\left(e^{j \kappa(i+1) \Delta x}-e^{j \kappa(i-1) \Delta x}\right)+a_{2}\left(e^{j \kappa(i+2) \Delta x}-e^{j \kappa(i-2) \Delta x}\right) \\
+a_{3}\left(e^{j \kappa(i+3) \Delta x}-e^{j \kappa(i-3) \Delta x}\right)+d_{0} e^{j \kappa i \Delta x}+d_{1}\left(e^{j \kappa(i+1) \Delta x}+e^{j \kappa(i-1) \Delta x}\right) \\
+d_{2}\left(e^{j \kappa(i+2) \Delta x}+e^{j \kappa(i-2) \Delta x}\right)+d_{3}\left(e^{j \kappa(i+3) \Delta x}+e^{j \kappa(i-3) \Delta x}\right)
\end{array}\right] u=0
$$

Dividing out the common factor $e^{j \text { jiax }}$ results in

$$
\frac{d u}{d t}+\frac{c}{\Delta x}\left[\begin{array}{c}
a_{1}\left(e^{j \kappa \Delta x}-e^{-j \kappa \Delta x}\right)+a_{2}\left(e^{j 2 \kappa \Delta x}-e^{-j 2 \kappa \Delta x}\right)+a_{3}\left(e^{j \kappa \Delta x}-e^{-j 3 \kappa \Delta x}\right) \\
+d_{0}+d_{1}\left(e^{j \kappa \Delta x}+e^{-j \kappa \Delta x}\right)+d_{2}\left(e^{j 2 \kappa \Delta x}+e^{-j 2 \kappa \Delta x}\right)+d_{3}\left(e^{j \kappa \Delta x}+e^{-j 3 \kappa \Delta x}\right)
\end{array}\right] u=0
$$

Recalling that $e^{\rho^{\theta}}-e^{-\rho \theta}=2 j \sin \theta$ and $e^{\rho \theta}+e^{-\rho \theta}=2 \cos \theta$ leads to 


$$
\frac{d u}{d t}+\frac{c}{\Delta x}\left[\begin{array}{c}
2 j\left(a_{1} \sin \kappa \Delta x+a_{2} \sin 2 \kappa \Delta x+a_{3} \sin 3 \kappa \Delta x\right) \\
+d_{0}+2\left(d_{1} \cos \kappa \Delta x+d_{2} \cos 2 \kappa \Delta x+d_{3} \cos 3 \kappa \Delta x\right)
\end{array}\right] u=0
$$

This can be written as

$$
\frac{d u}{d t}=-j c \kappa^{*} u=\lambda u
$$

where $\lambda=-j c \kappa^{*}$, and $\kappa^{*}$ is the modified wavenumber which is given by the equation

$$
\begin{aligned}
z^{\bullet}=\kappa^{\bullet} \Delta x=2 & \left(a_{1} \sin z+a_{2} \sin 2 z+a_{3} \sin 3 z\right) \\
& -j\left(d_{0}+2\left(d_{1} \cos z+d_{2} \cos 2 z+d_{3} \cos 3 z\right)\right)
\end{aligned}
$$

where $z=\kappa \Delta x$ is the nondimensional wavenumber. The modified wavenumber depends on the numerical scheme that is used to approximate the spatial derivative, the grid resolution, and $z$. Errors can be written more concisely using $z^{\circ}(z)$, the modified nondimensional wavenumber.

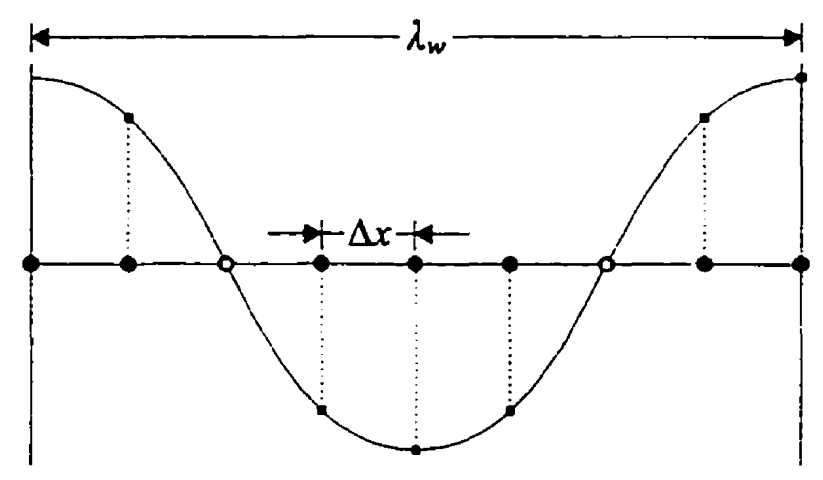

Figure 4: Example of harmonic signal resolved by a grid using 8 PPW

In the limit as $z$ approaches zero, the value of the modified wavenumber approaches the value of the wavenumber, provided that the numerical schemes are at least first-order accurate. This is easy to explain in terms of the number of grid points used to resolve a wave, known as the points per wavelength (PPW) (see Figure 4). The PPW, $n_{\lambda}$, for a harmonic signal with a wavelength $\lambda_{w}$, resolved by a uniform grid with spacing $\Delta x$, can be defined using the following relation

$$
n_{\lambda}=\frac{\lambda_{w}}{\Delta x}
$$


Noting that the frequency of the signal is given by

$$
\frac{\omega}{2 \pi}=\frac{c}{\lambda_{w}}
$$

and using ( 3.21 ), $z$ can be related to the PPW:

$$
z=\frac{2 \pi}{n_{\lambda}}
$$

The above relation shows that the nondimensional wavenumber is inversely proportional to the PPW. This means that as $z$ approaches zero, the PPW approaches infinity. When this happens, the numerical discretization approaches the continuous problem, and any finite-difference method which is at least first-order accurate will become exact.

It should also be noted that a lower limit on the PPW has to be set. To obtain any information about a sinusoidal signal it must be resolved by a grid with at least two PPW. At this lower limit the signal will be approximated by a saw-tooth wave, which is the result of using only the endpoints and the center point of the grid in Figure 4. This gives an upper limit of $\pi$ for the value of the nondimensional wavenumber.

For the more general two-dimensional case, consider the following partial differential equation

$$
\frac{\partial U}{\partial t}+c\left(\cos \theta \frac{\partial U}{\partial x}+\sin \theta \frac{\partial U}{\partial y}\right)=0
$$

This equation governs the propagation of a plane wave which moves at the speed $c$ in the direction defined by the vector making an angle of $\theta$ with the $x$-axis. Performing the above analysis on this equation again leads to the ODE given in ( 3.26 ), except now the modified wave number is also dependent on the direction of propagation. If the same finite-difference scheme is used to approximate the $x$ and $y$ derivatives on a square grid $(\Delta x=\Delta y)$, the twodimensional version of the modified nondimensional wavenumber can be determined from

$$
z_{2 \mathrm{D}}^{*}(z, \theta)=\kappa_{2 \mathrm{D}}^{*} \Delta x=\cos \theta z_{1 \mathrm{D}}^{*}(z \cos \theta)+\sin \theta z_{\mathrm{ID}}^{*}(z \sin \theta)
$$

where $z_{\mathrm{ID}}^{*}=\kappa_{\mathrm{ID}}^{*} \Delta x$ is determined using the one-dimensional analysis.

To add the effects of the time-stepping method to the error analysis, consider equation ( 3.26 ) which is rewritten here as 


$$
\frac{d u}{d t}=\lambda u=f(u, t)
$$

Noting the above value of the function $f$, the time-marching method can be used to solve for the unknown variable $u$. Direct substitution of $f$ into ( 3.12 ) gives

$$
\begin{aligned}
& u_{n+\alpha_{1}}^{(1)}=u_{n}+\lambda h \alpha_{1} u_{n} \\
& u_{n+\alpha_{2}}^{(2)}=u_{n}+\lambda h \alpha_{2} u_{n+\alpha_{1}}^{(1)} \\
& u_{n+\alpha_{3}}^{(3)}=u_{n}+\lambda h \alpha_{3} u_{n+\alpha_{2}}^{(2)} \\
& u_{n+\alpha_{4}}^{(4)}=u_{n}+\lambda h \alpha_{4} u_{n+\alpha_{3}}^{(3)} \\
& u_{n+\alpha_{3}}^{(s)}=u_{n}+\lambda h \alpha_{5} u_{n+\alpha_{4}}^{(4)} \\
& u_{n+1}=u_{n}+\lambda h u_{n+\alpha_{3}}^{(s)}
\end{aligned}
$$

A solution for $u_{n+1}$ in terms of $u_{n}$ can easily be obtained using back substitution:

$$
u_{n+1}=u_{n}+\lambda h\left(u_{n}+\lambda h \alpha_{5}\left(u_{n}+\lambda h \alpha_{4}\left(u_{n}+\lambda h \alpha_{3}\left(u_{n}+\lambda h \alpha_{2}\left(u_{n}+\lambda h \alpha_{1} u_{n}\right)\right)\right)\right)\right)
$$

which can be written as

$$
u_{n+1}=\sigma u_{n}
$$

where

$$
\sigma=1+\lambda h+\beta_{2}(\lambda h)^{2}+\beta_{3}(\lambda h)^{3}+\beta_{4}(\lambda h)^{4}+\beta_{5}(\lambda h)^{5}+\beta_{6}(\lambda h)^{6}
$$

and

$$
\begin{aligned}
& \beta_{2}=\alpha_{5}, \quad \beta_{3}=\alpha_{5} \alpha_{4}, \quad \beta_{4}=\alpha_{5} \alpha_{4} \alpha_{3}, \\
& \beta_{5}=\alpha_{5} \alpha_{4} \alpha_{3} \alpha_{2}, \quad \beta_{6}=\alpha_{5} \alpha_{4} \alpha_{3} \alpha_{2} \alpha_{1}
\end{aligned}
$$

The solution to ( 3.35 ) is given by

$$
u_{n}=u_{0} \sigma^{n}
$$

The solution is in terms of $\sigma$, which is the root of the characteristic polynomial of the timemarching method. This root is dependent on the method used and the product $\lambda h$. In general, a method may have more than one characteristic root. The time-marching methods considered in this work have a single root, which greatly simplifies the analysis.

The exact solution to equation ( 3.26 ) is

$$
u(t)=u_{0} e^{\lambda t}
$$


Noting that $t=n h$ and comparing the above two equations, the following result is obtained

$$
\sigma \approx e^{\lambda \hbar}
$$

A time-marching method's order of accuracy, when applied to a linear homogeneous ODE, can be determined by finding out how many terms of $\sigma$ match the Taylor series expansion of $e^{i h}$. From ( 3.36 ) it is clear that the family of time-marching methods being considered is at least first-order accurate, and at most sixth-order accurate, for linear homogeneous ODEs. The second-order scheme discussed in the previous section gives $\beta_{2}=1 / 2$, and the sixth-order scheme, for linear homogeneous ODEs, gives $\beta_{m}=1 / m$ for $m=2 \ldots 6$, as expected.

To determine the value of the characteristic root when the fully-discrete approximation is used, one first determines $z_{1 D}^{*}(z)$ for the spatial finite-difference scheme as given by equation ( 3.27). Given this, $z_{2 \mathrm{D}}(z, \theta)$ is easily obtained using ( 3.32 ). To calculate $\sigma$, the product $\lambda h$ must be obtained, which equation ( 3.26 ) gives as

$$
\lambda h=-j c \kappa_{2 \mathrm{D}}^{*} h=-j c \frac{\Delta t}{\Delta x} z_{2 \mathrm{D}}^{*}
$$

Noting that the Courant number is given by

$$
C=c \frac{\Delta t}{\Delta x}
$$

( 3.41 ) can be rewritten as

$$
\lambda h(z, \theta, C)=-j C z_{2 \mathrm{D}}^{*}(z, \theta)
$$

Using this and (3.36), $\sigma(\lambda h)=\sigma(z, \theta, C)$ can be obtained.

Using equation ( 3.40 ) and the exact value of $\lambda$ it is clear that

$$
\sigma=\sigma_{r}+j \sigma_{1} \approx e^{-j \alpha x h}=e^{-j c z}
$$

where $\sigma_{r}$ and $\sigma_{t}$ are the real and imaginary parts of the characteristic root. The above equation shows that the exact value of $\sigma$ has a magnitude of unity and a phase angle of $-C z$. These relations lead to definitions for the local amplitude error,

$$
e r_{a}=|\sigma|-1
$$

and the normalized local phase error, 


$$
e r_{p}=1+\frac{1}{C z} \tan ^{-1}\left(\frac{\sigma_{i}}{\sigma_{r}}\right)
$$

of the numerical approximation. These errors depend on $z, \theta$, and $C$, and they generally increase as $z$ increases.

Plots of the amplitude and phase errors as a function of $z$ over the range 0 to $\pi$ are shown in Figures 5 and 6 for some of the schemes that are discussed in the previous section. The errors shown are for a second-order central-difference scheme coupled with a third-order filter with fourth-order time-marching (C2), a fourth-order central-difference scheme coupled with a third-order filter with fourth-order time-marching (C4), and the maximum-order scheme which has a sixth-order central-difference scheme coupled with a fifth-order filter with sixth-order time-marching (MO). The strength of the filter for the $\mathrm{C} 2$ and $\mathrm{C} 4$ schemes is $d_{0}=0.06$ and for the MO scheme is $d_{0}=0.1$. These values are chosen to help ensure the stability of the methods while keeping the overall effect on the errors minimal. For the fourthorder time-marching method the characteristic root, $\sigma$, can be obtained by using $\beta_{m}=1 / m$, $m=2 \ldots 4$, and $\beta_{5}=\beta_{6}=0$ in equation ( 3.36 ).

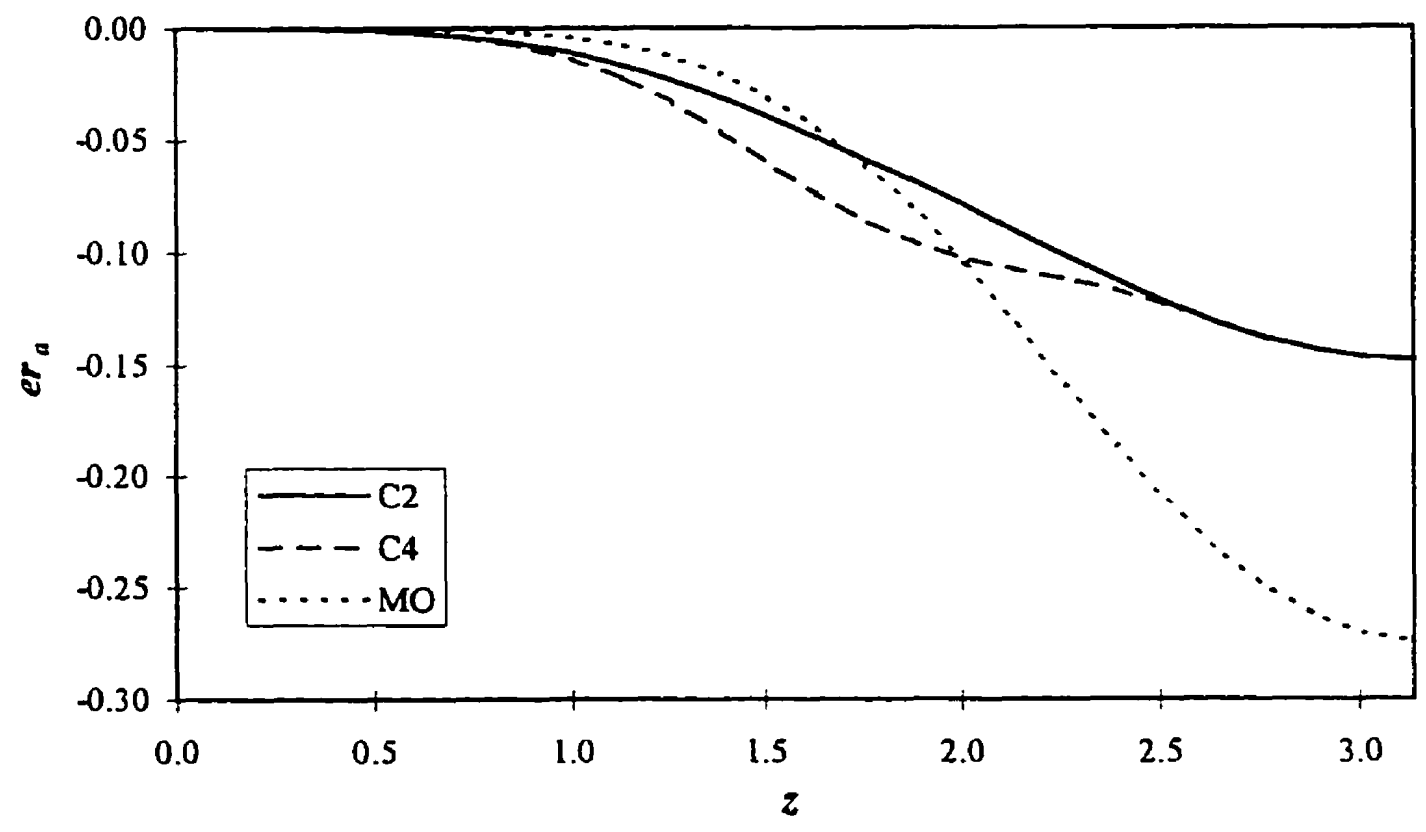

Figure 5: Local amplitude errors with $\theta=0^{\circ}$ and $C=1$ for second-order (C2), fourth-order (C4), and sixth-order (MO) schemes 


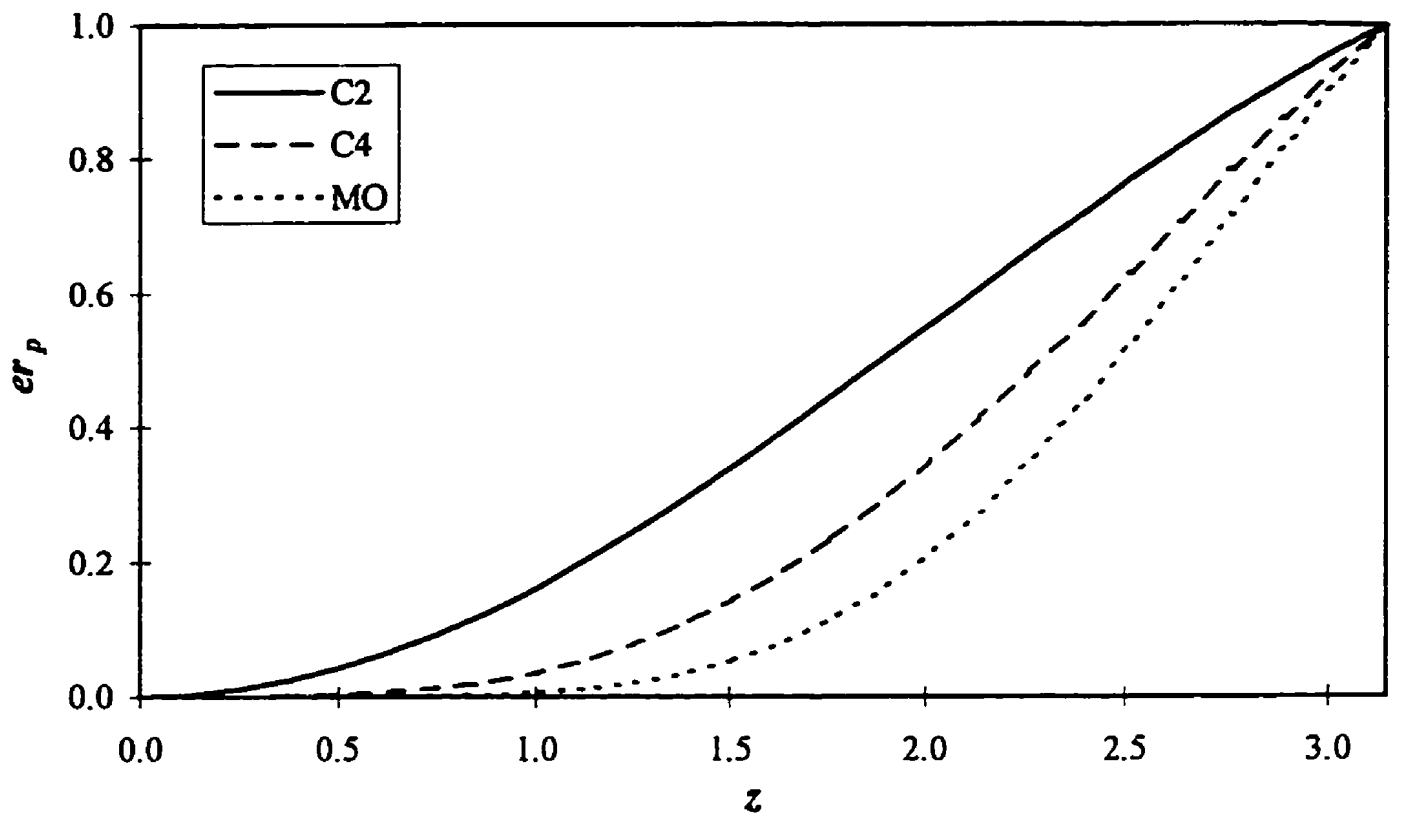

Figure 6: Normalized local phase errors with $\theta=0^{\circ}$ and $C=1$ for second-order (C2), fourth-order (C4), and sixth-order (MO) schemes

Consider the total errors that will result after a scheme has been used over a number of time-steps, $n_{t}$. The global amplitude error is given by

$$
E r_{a}=\left.|1-| \sigma\right|^{n} \mid
$$

and the global phase error is

$$
E r_{p}=n_{t}\left|C z+\tan ^{-1}\left(\frac{\sigma_{i}}{\sigma_{r}}\right)\right|
$$

These errors can be used in a very effective method for evaluating a scheme's efficiency. This is done by determining the PPW, $n_{k}$, required to propagate a wave a given number of wavelengths of travel, $n_{w}$, while keeping both $E r_{a}$ and $E r_{p}$ under 0.1 ; see, for example, [44]. Recalling that ( 3.30 ) expresses $z$ in terms of the PPW, and by noting that

$$
n_{t}=\frac{n_{\lambda} n_{w}}{C}
$$

the global errors can easily be expressed as functions of both $n_{\lambda}$ and $n_{w}$. Setting the errors equal to 0.1 implicitly defines the PPW needed to travel a given number of wavelengths. In general, there may be more than one solution for these equations. If this is the case, care must be taken to ensure that the largest value of $n_{\lambda}$ is used for a given $n_{w}$. Once the PPW values are 
found for the global amplitude error and the global phase error, taking the maximum of the two gives the required result.

Figure 7 shows a plot of the PPW required to propagate a wave a given number of wavelengths for the $\mathrm{C} 2, \mathrm{C} 4$, and $\mathrm{MO}$ schemes. This figure presents the relevant information in a very concise way, easily allowing a quick comparison of the merits of each scheme. For example, if a wave is to be numerically propagated 10 wavelengths while keeping the global errors bounded by $0.1, \mathrm{C} 2$ needs a grid with a resolution of about $60 \mathrm{PPW}, \mathrm{C} 4$ needs about $15 \mathrm{PPW}$, and MO needs only $8 \mathrm{PPW}$. This means that C4 can use one fourth the number of nodes for a one-dimensional problem as $\mathrm{C} 2$ to achieve the same accuracy, a factor of 16 less in two dimensions, and a factor of 64 less in three dimensions. On the other hand, $\mathrm{C} 4$ needs to use about twice as many nodes as MO to obtain the same error levels for a one-dimensional problem, 4 times as many nodes in two dimensions, and 8 times as many nodes in three dimensions.

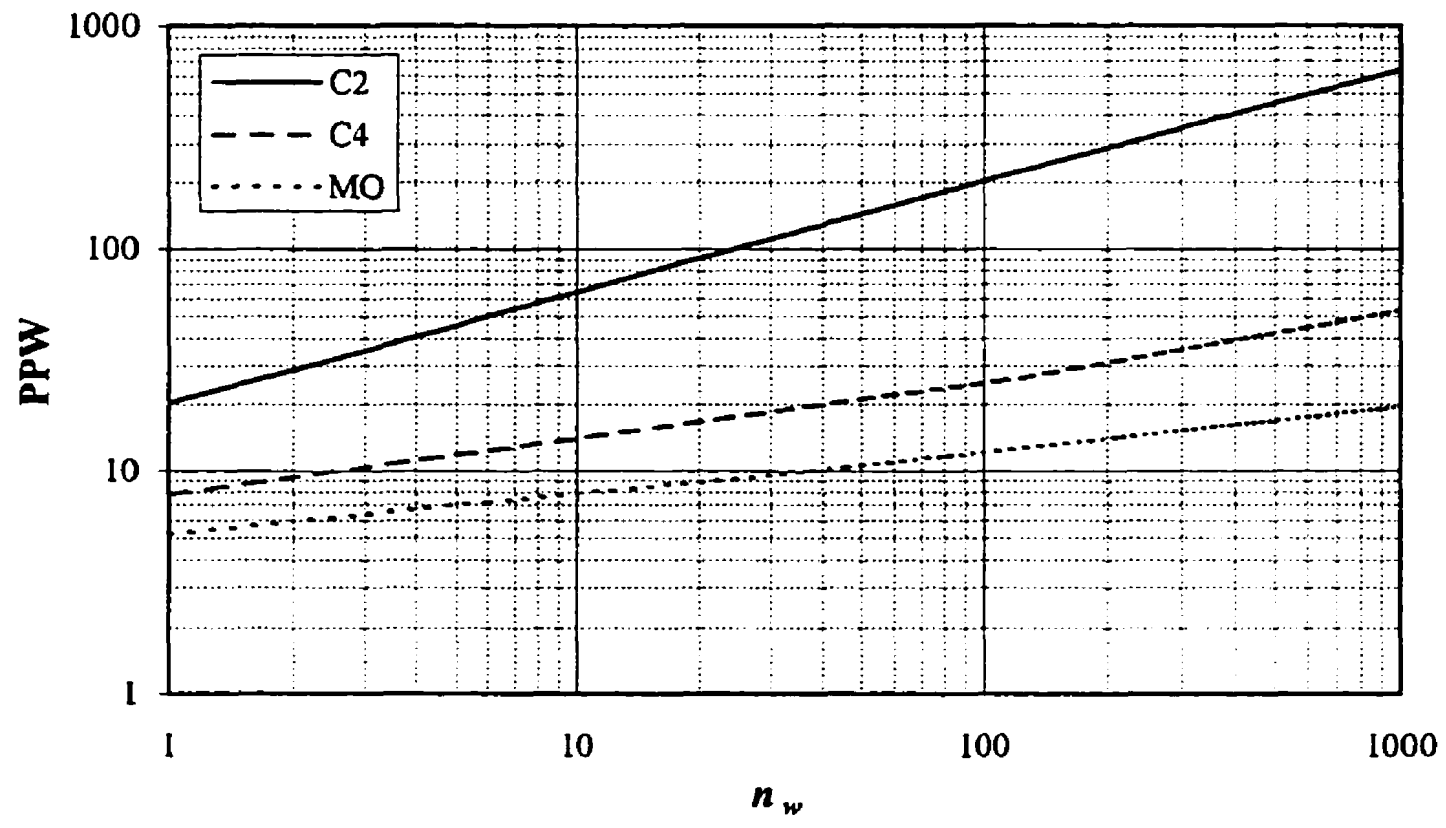

Figure 7: PPW required to propagate a wave $n_{w}$ wavelengths while keeping the global errors less than 0.1 with $\theta=0^{\circ}$ and $C=1$ for second-order (C2), fourth-order (C4), and sixth-order (MO) schemes

The errors presented in this section do not take into account the impact of the NBSs which must be used near the surface of scattering objects, or grid nonuniformity. Furthermore, 
although the PPW requirements give an accurate picture of memory usage, CPU expense is also dependent on the cost per node of the methods. The results of the numerical tests, presented in Chapter 5, show the relative efficiencies of these schemes for more realistic problems.

\subsubsection{Optimized Schemes}

Equations ( 3.1 ), ( 3.2 ) and ( 3.12 ) define a set of spatial operators and a time-marching method which can be used to model PDEs. This chapter has already defined a number of methods for which the coefficients in the schemes were found by maximizing the formal order of accuracy, for a given computational stencil size, using Taylor series expansions. A number of papers $[15,18,26,42,44,73,74,95]$ have discussed determining the coefficients of a scheme in order to obtain better error properties for a range of wavenumbers, as opposed to maximizing the formal order. The rest of this section outlines the development of schemes, discussed in $[30,89,91,95]$, which are optimized to produce amplitude and phase errors that are minimized over a range of wavenumbers.

Consider the spatial operators given by ( 3.1 ) and ( 3.2 ). By constraining these operators to be at least first-order accurate a family of schemes is obtained in which there are four free parameters (see the schemes in Tables I and 2). If these operators are used in conjunction with the time-marching method given by ( 3.12 ), constrained to be second-order accurate, another four free parameters are obtained. Using Fourier error analysis the local amplitude error ( 3.45 ) and local normalized phase error ( 3.46 ) can be found for this family of numerical schemes. These errors are dependent on the 8 free parameters $a_{2}, a_{3}, d_{2}, d_{3}, \beta_{3}, \beta_{4}, \beta_{5}$, and $\beta_{6}$, as well as the strength of the filter $d_{0}$, and the value of $z, \theta$, and $C$. Equation ( 3.37 ) allows the $\alpha_{i}$ to be expressed in terms of the $\beta_{i}$ which explicitly appear in the error equations. The maximum-order scheme (MO), which is determined by choosing the free parameters in order to minimize the truncation error, is a member of this family of schemes.

Lele [42] noted that the free parameters in his compact central-difference operators could be used to force the phase error to be zero at a number of values of $z$. Determining the free parameters by selectively choosing where the zeros of the error were placed, he generated spatial operators which had error bounds that were much smaller, over a given range of wavenumbers, than those obtained by finding the parameters which minimized the truncation error. 
Using this idea, the 8 free parameters in the scheme discussed above can be used to force the errors to be zero at 8 different places. To find schemes for which the amplitude and phase errors are roughly of equal value, the parameters are used to pin the amplitude error to zero four times, and the phase error to zero four times. The pinning locations can be optimized in order to minimize the maximum values of the amplitude and phase errors for a given range of wavenumbers.

Before finding the coefficients of a scheme, the minimum number of PPW a given problem is going to need must be decided. This can be determined by having some information about the size of the problem domain, the smallest expected wavelength that will be introduced during the simulation, and the amount of physical computer memory available. Once the value of the minimum PPW required is known, it will define the range of nondimensional wavenumbers over which the errors will be optimized. For example, if a scheme is required in which the smallest expected wavelength is to be resolved by $10 \mathrm{PPW}$, the coefficients of the scheme will be determined by optimizing over the range $z \in[0, \pi / 5]$. Also, before the optimization can begin, the strength of the filter must be set. The schemes discussed in this document have a value of $d_{0}$ chosen to help insure numerical stability, while trying to minimize the impact on the errors.

When performing the optimization, care must be taken in defining the choice of the error function which is to be minimized. The aim is to minimize the maximum phase and amplitude errors over a known range of nondimensional wavenumbers. For problems in two or more dimensions one must also consider the errors' dependence on the direction of travel. Another factor that arises, when using spatial grids which have variable node spacing, is that when a constant time-step is used the Courant number will vary spatially. For this reason the errors' dependence on the Courant number must also be taken into account. If these factors are ignored, one can develop schemes which exhibit outstanding properties for given values of $\theta$ and $C$, but changing either of these values by a small amount can degrade the method's performance significantly. For example, consider the method, whose errors are shown in Figure 8, which is optimized for $10 \mathrm{PPW}$ using a filter strength of 0.5 while holding $\theta=0^{\circ}$ and $C=1$. Changing the direction of propagation from an angle of $0^{\circ}$ to $45^{\circ}$ causes the maximum error, over the range of nondimensional wavenumbers, to increase by a factor of about $10^{4}$. 
The maximum errors are also increased by a factor of about $10^{4}$ by changing the Courant number from 1 to 0.5 , which represents a factor of two stretch in the spatial grid.

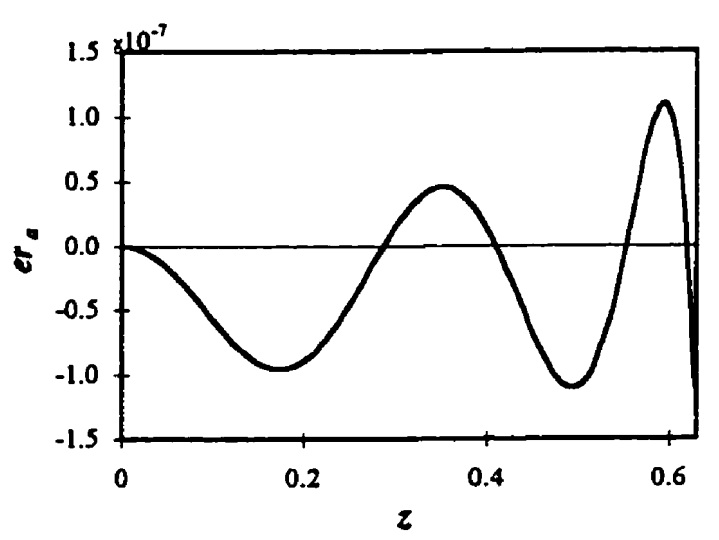

a)

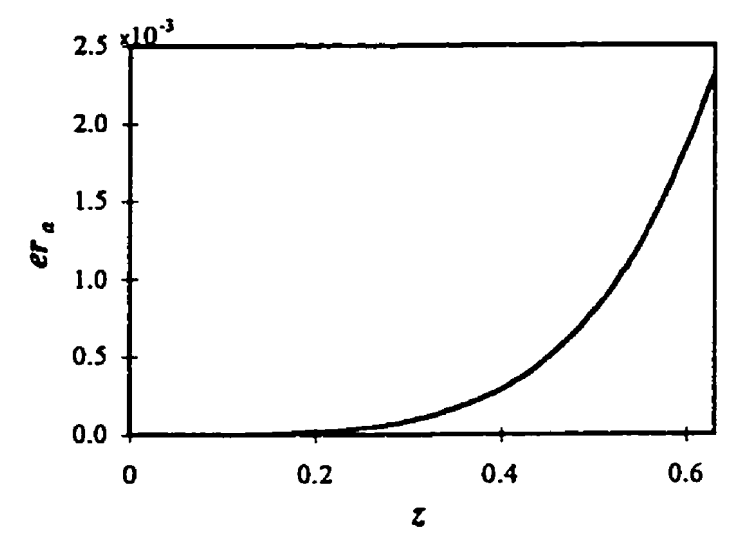

c)

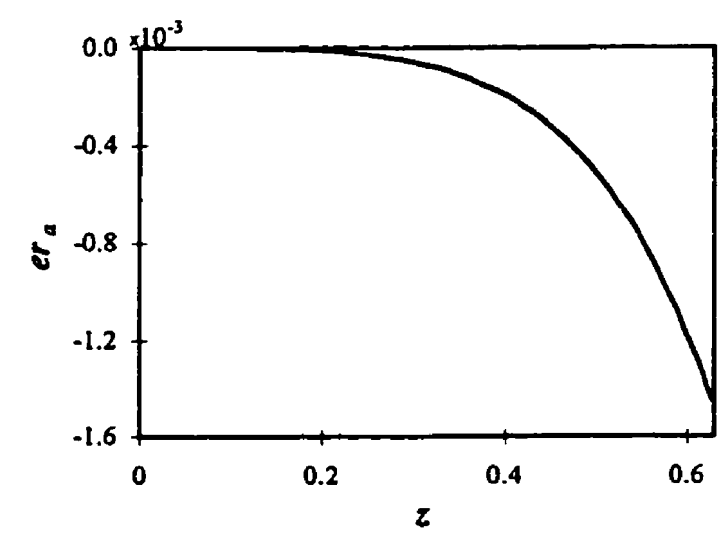

e)

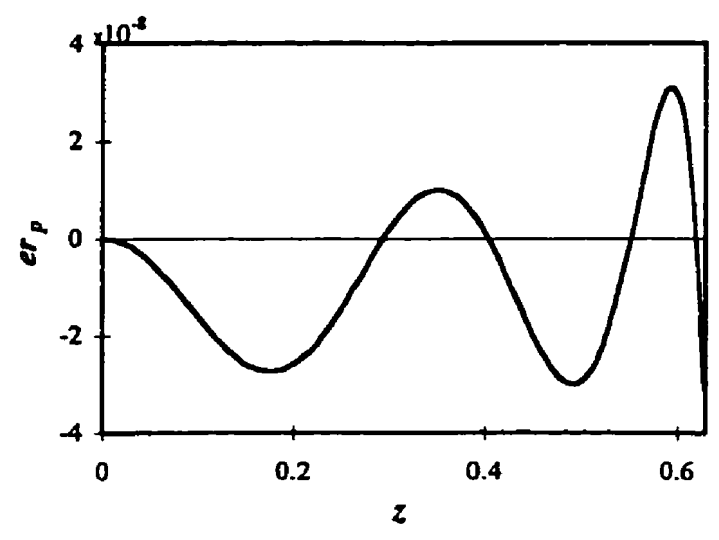

b)

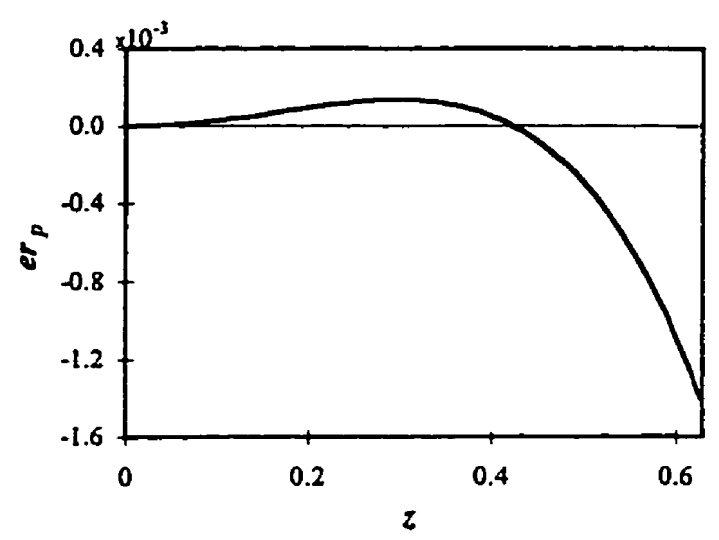

d)

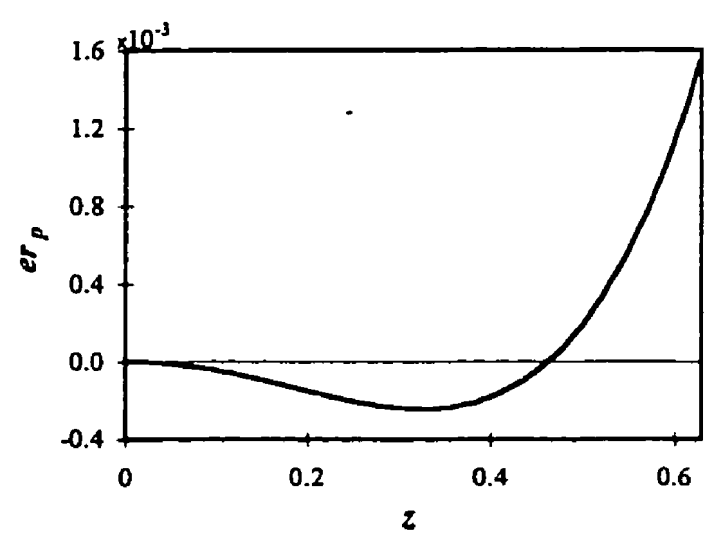

f)

Figure 8: Errors for scheme optimized for $\theta=0^{\circ}$ and $C=1$, a) and b) amplitude and phase errors for $\theta=0^{\circ}$ and $\left.C=1, c\right)$ and d) amplitude and phase errors for $\theta=45^{\circ}$ and $C=1, \mathrm{e})$ and $\mathrm{f}$ ) amplitude and phase errors for $\theta=0^{\circ}$ and $C=1 / 2$. 
The above example shows that care must be taken when optimizing the schemes in order to obtain a method which exhibits enough flexibility to be used for multi-dimensional problems on general curvilinear grids. A useful method for finding such flexible schemes is to optimize the spatial operator first, using the four available parameters to pin the spatial errors, and then optimizing the entire scheme using the four parameters available from the timemarching scheme. This optimization is performed while holding the value of $\theta$ constant at zero degrees. Optimizing the spatial scheme by itself is the same as finding an optimum scheme when the time-step is very small compared to the grid spacing, that is, having a very small Courant number. When this is the case, the error due to the time-marching method is negligible and the spatial errors can be considered by themselves. The fully optimized method, found using this spatial scheme in combination with the time-marching method at a Courant number of one, gives very good error properties over the range of Courant numbers from zero to one. A benefit of finding a method in this way is that it also has very good error properties for all directions of travel.

To perform an optimization on the spatial scheme, phase and amplitude errors must first be defined for the semi-discrete operator. Consider the Fourier analysis performed in the previous section. The exact solution for the semi-discrete problem is given by equation ( 3.19 ) and is restated here

$$
u(t)=e^{-\jmath \alpha t}=e^{-\jmath C z}
$$

Approximating the spatial derivatives using finite-differences results in the following estimate for the solution

$$
u(t)=e^{-ر z_{i}^{*}}=e^{c_{i}^{*}} e^{-j c z_{i}^{*}}
$$

where $z_{r}^{*}$ is the real part and ${z_{i}^{*}}^{*}$ is the imaginary part of the modified nondimensional wavenumber. By comparing the above two equations, the following definitions for the semi-discrete local amplitude and normalized local phase errors can be defined:

$$
\begin{aligned}
& e r_{a}^{s d}=e^{c z_{i}^{*}}-1 \\
& e r_{p}^{s d}=1-\frac{z_{r}^{*}}{z}
\end{aligned}
$$

The above errors are functions of the nondimensional wavenumber, $z$, and the direction of 
propagation, $\theta$. The amplitude error is also a function of the Courant number, $C$. Strictly speaking, the Courant number should not be a factor in the error calculations because a timemarching method is not being considered. This definition for the amplitude error is only used as a tool in the first step for finding an optimized fully-discrete scheme. Very good schemes have been found by setting the Courant number to one in this amplitude error.

A number of schemes have been developed using the method discussed above. Three of these schemes will now be considered. These schemes are optimized in order to obtain minimum errors over different nondimensional wavenumber ranges. The ranges are defined by considering schemes which are optimized for problems with grids that resolve the expected highest frequency components by at least 10 PPW, 7.5 PPW, and 5 PPW. The coefficients of these schemes can be found in Appendix $\mathrm{C}$.

Figures 9 and 10 compare the local amplitude error and the local normalized phase error of the above optimized schemes to the MO scheme. The scheme optimized for $10 \mathrm{PPW}$ is referred to as 010, the scheme optimized for 7.5 PPW is referred to as 07.5, and the scheme optimized for 5 PPW is referred to as O5. The figures display the errors for a Courant number

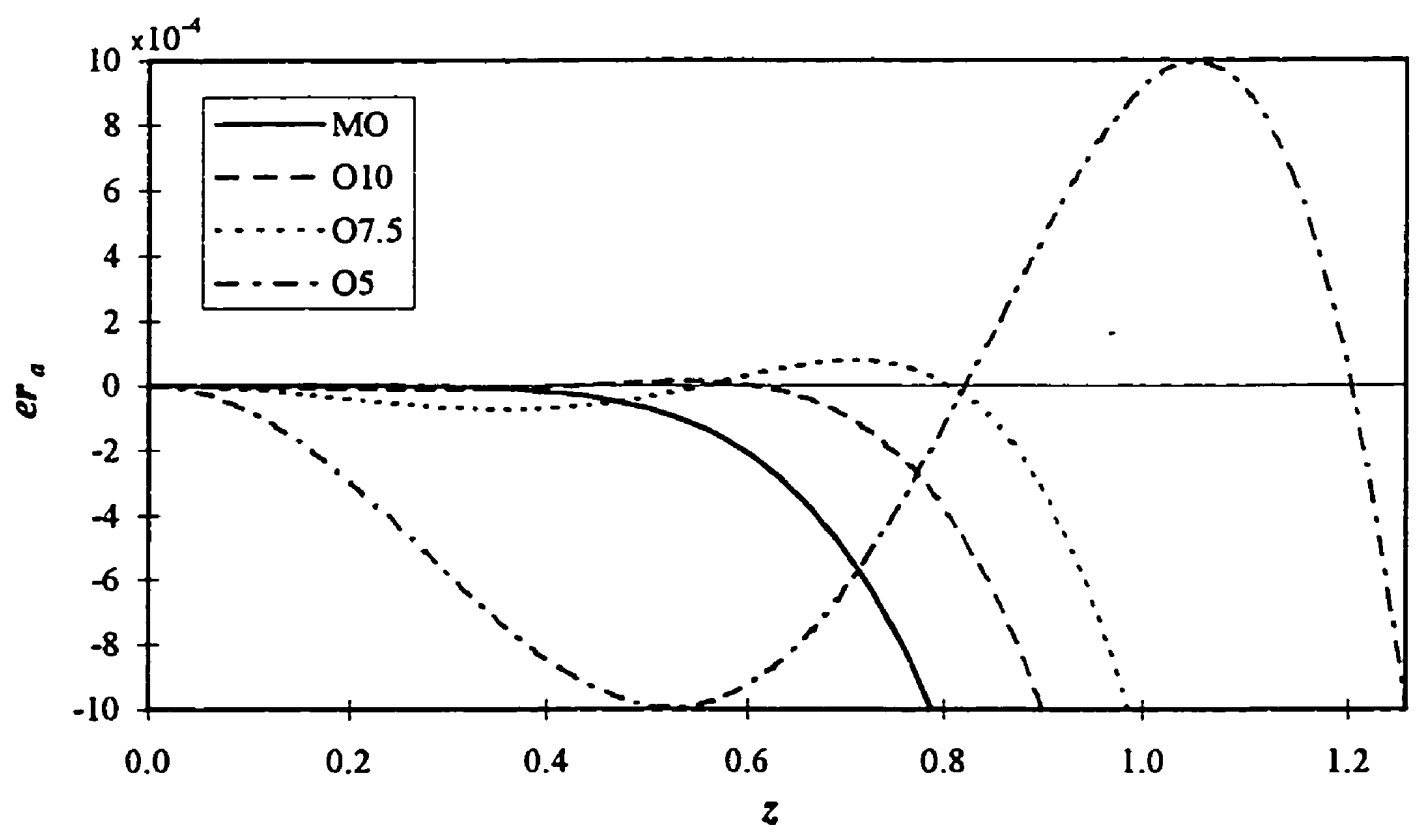

Figure 9: Local amplitude errors with $\theta=0^{\circ}$ and $C=1$ for $\mathrm{MO}, 010,07.5$, and O5 schemes 


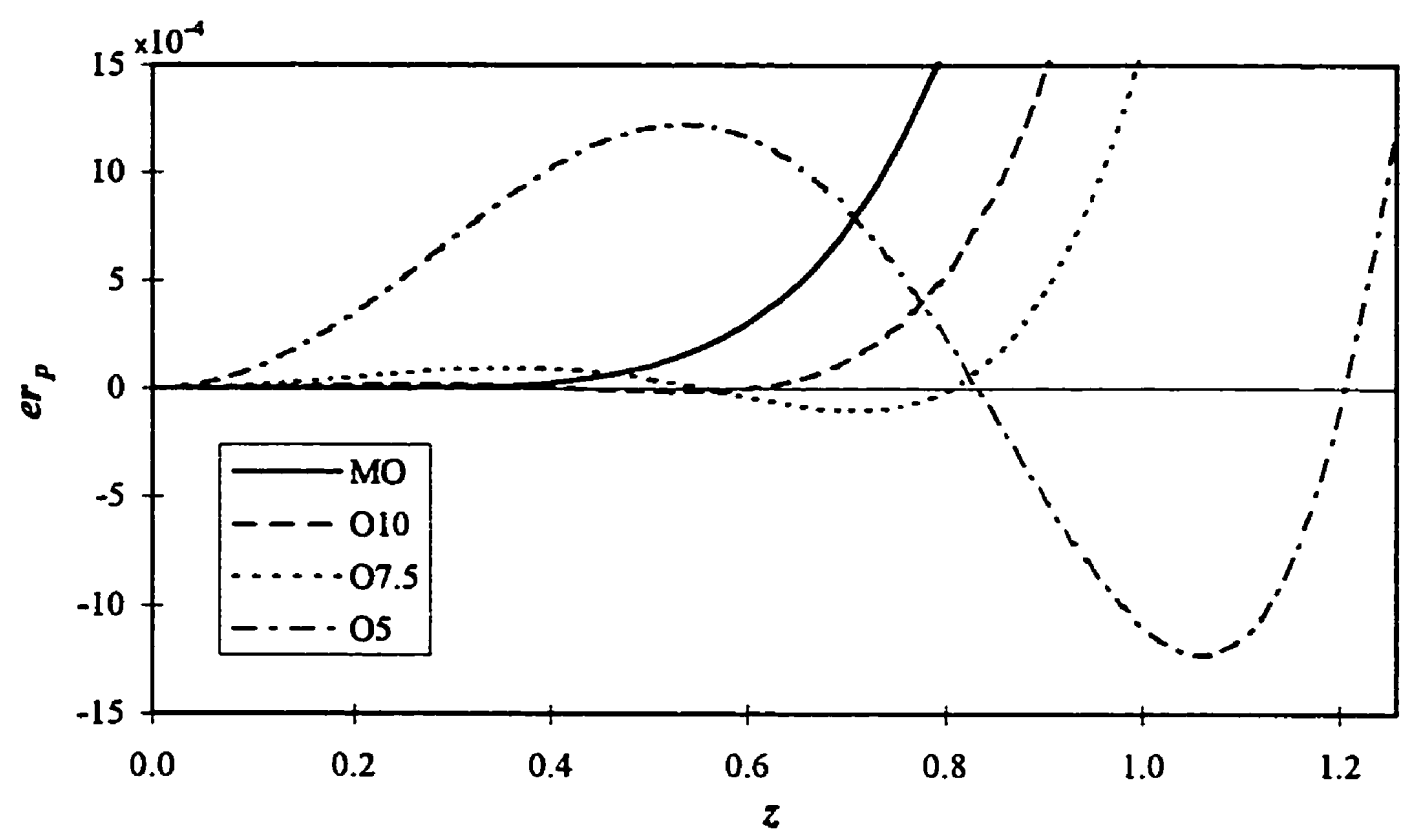

Figure 10: Normalized local phase errors with $\theta=0^{\circ}$ and $C=1$ for $\mathrm{MO}, 010$, 07.5, and 05 schemes

of one and an incident angle of zero degrees, concentrating on the range of nondimensional wavenumbers associated with resolving a problem with at least $5 \mathrm{PPW}$. These figures clearly show the tradeoffs associated with optimizing the schemes more aggressively. Consider the O5 scheme, for values of the nondimensional wavenumber greater than 1 this scheme generates the smallest errors, but when $z$ falls below 0.7 all the other schemes produce smaller errors.

Figure 11 shows the PPW required to propagate a wave a given number of wavelengths of travel, while keeping the global errors less than 0.1 , using the optimized schemes and the maximum-order scheme. The effect of optimizing a scheme over a larger range of nondimensional wavenumbers (i.e. a smaller number of PPW) becomes very clear when inspecting this figure. The curve resulting from using the MO scheme appears to be relatively straight on this log-log plot. When using an optimized scheme, better results are obtained when the wavelengths of travel are small, but at some critical-point there is a discontinuous jump in the PPW requirements which results in a need for a much finer grid than that required by the MO scheme. By optimizing a scheme for smaller PPW requirements, the number of PPW required to propagate a wave for a short distance becomes smaller, but the critical-point occurs earlier. 
The critical-point for the 05 scheme occurs at about 8 wavelengths of travel. To propagate a wave 7 wavelengths the 05 scheme requires less than 5 PPW while the MO scheme needs more than $7 \mathrm{PPW}$. The critical-point for the 07.5 scheme occurs at just over 70 wavelengths of travel. To propagate a wave 70 wavelengths the 07.5 scheme requires around 7 PPW while the MO scheme needs more than 11 PPW (the O5 scheme requires over 200 PPW). The critical-point for the 010 scheme occurs at over 300 wavelengths of travel. To propagate a wave 300 wavelengths the 010 scheme requires less than 10 PPW while the MO scheme needs more than 15 PPW (the 07.5 and O5 schemes require over 140 PPW and 900 PPW respectively).

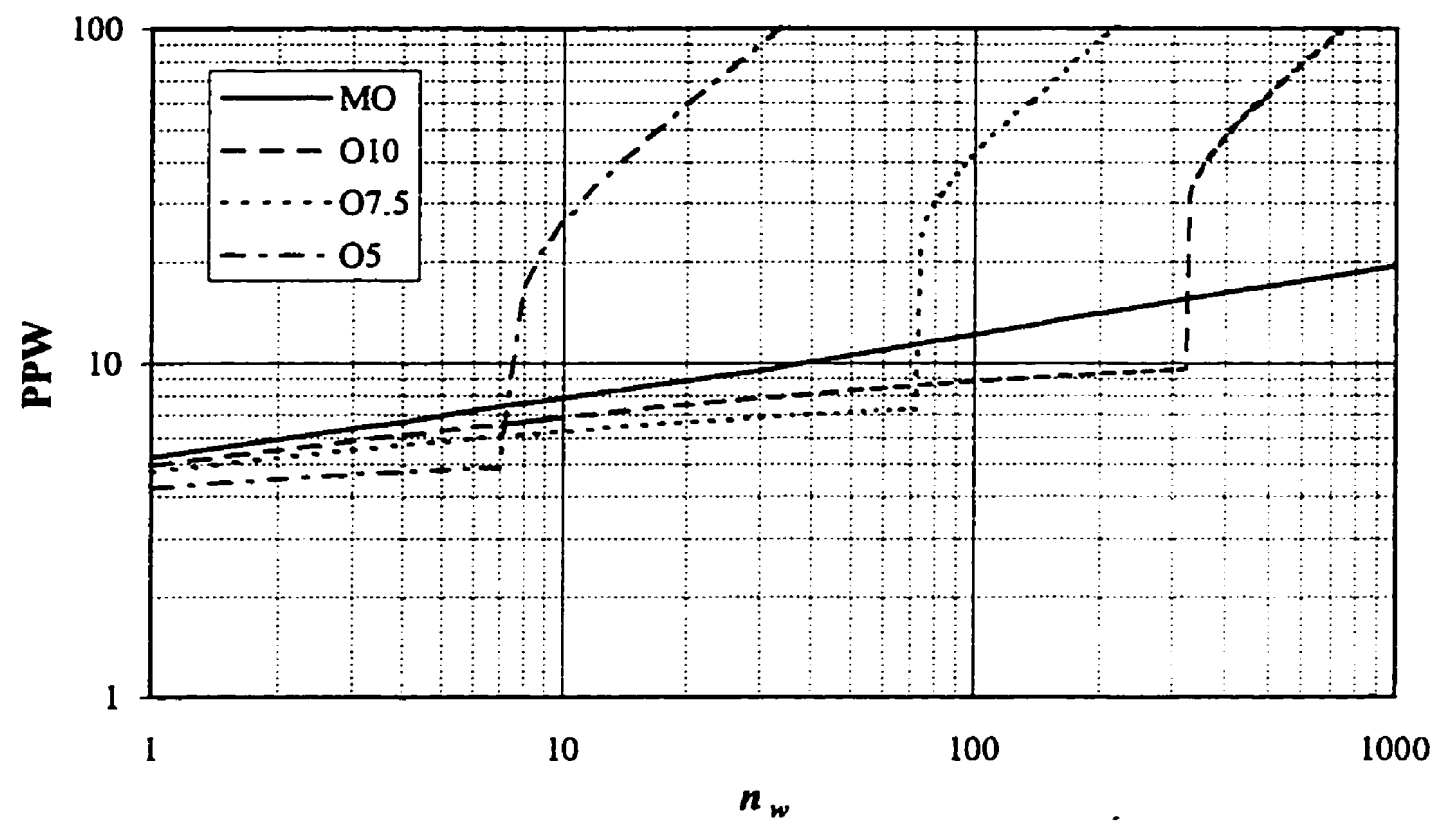

Figure 11: PPW required to propagate a wave $n_{w}$ wavelengths while keeping the global errors less than 0.1 with $\theta=0^{\circ}$ and $C=1$ for $\mathrm{MO}, 010,07.5$, and $\mathrm{O5}$ schemes

An optimized scheme should produce small errors for a range of Courant numbers and angles of incidence. The method for optimizing the schemes, discussed above, produces errors which exhibit these properties. For example, Figures 12 and 13 show the local amplitude error and local normalized phase error, respectively, for the $\mathrm{O} 10$ scheme at a number of different combinations of $C$ and $\theta$. The range of nondimensional wavenumbers shown in the figures is 


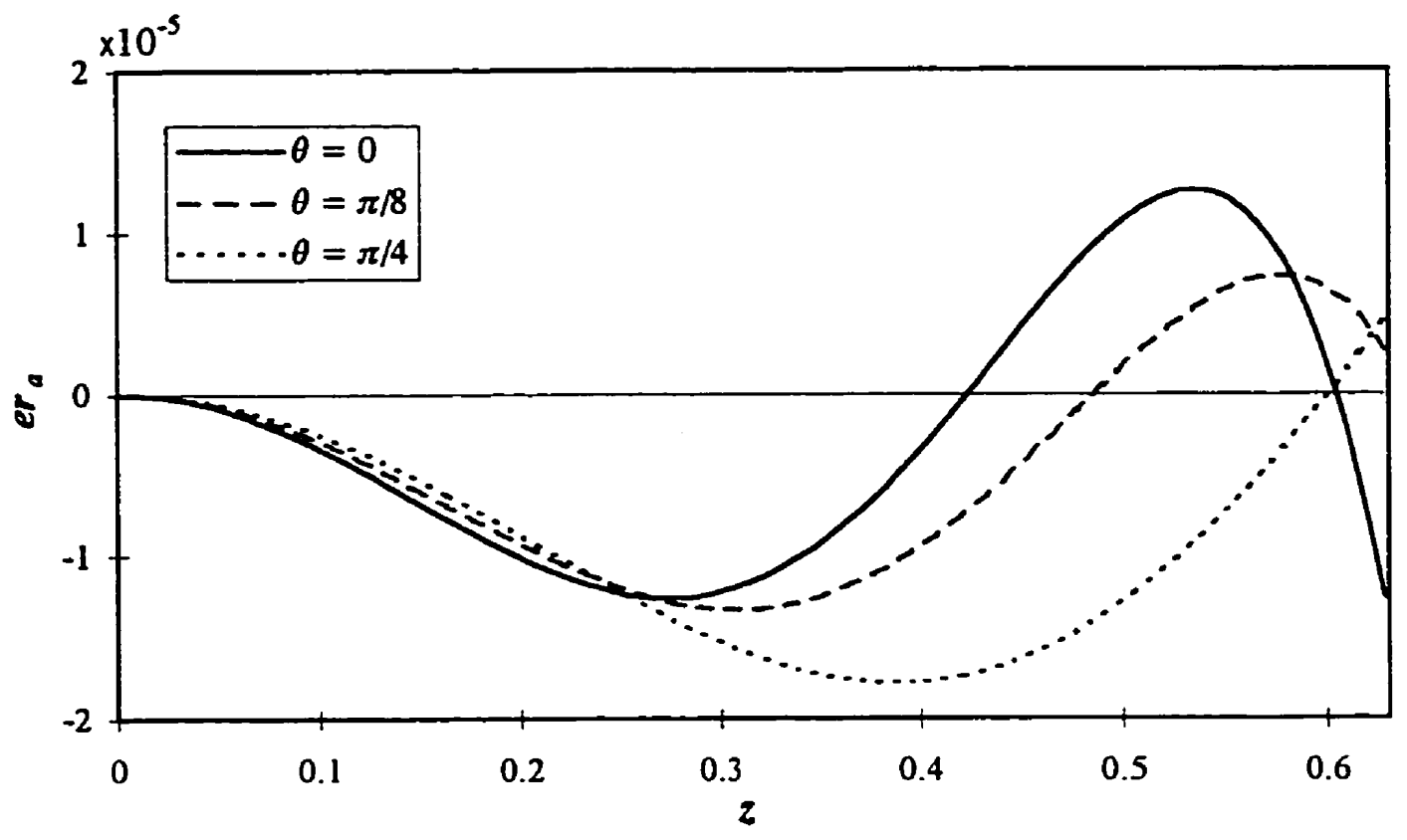

a)

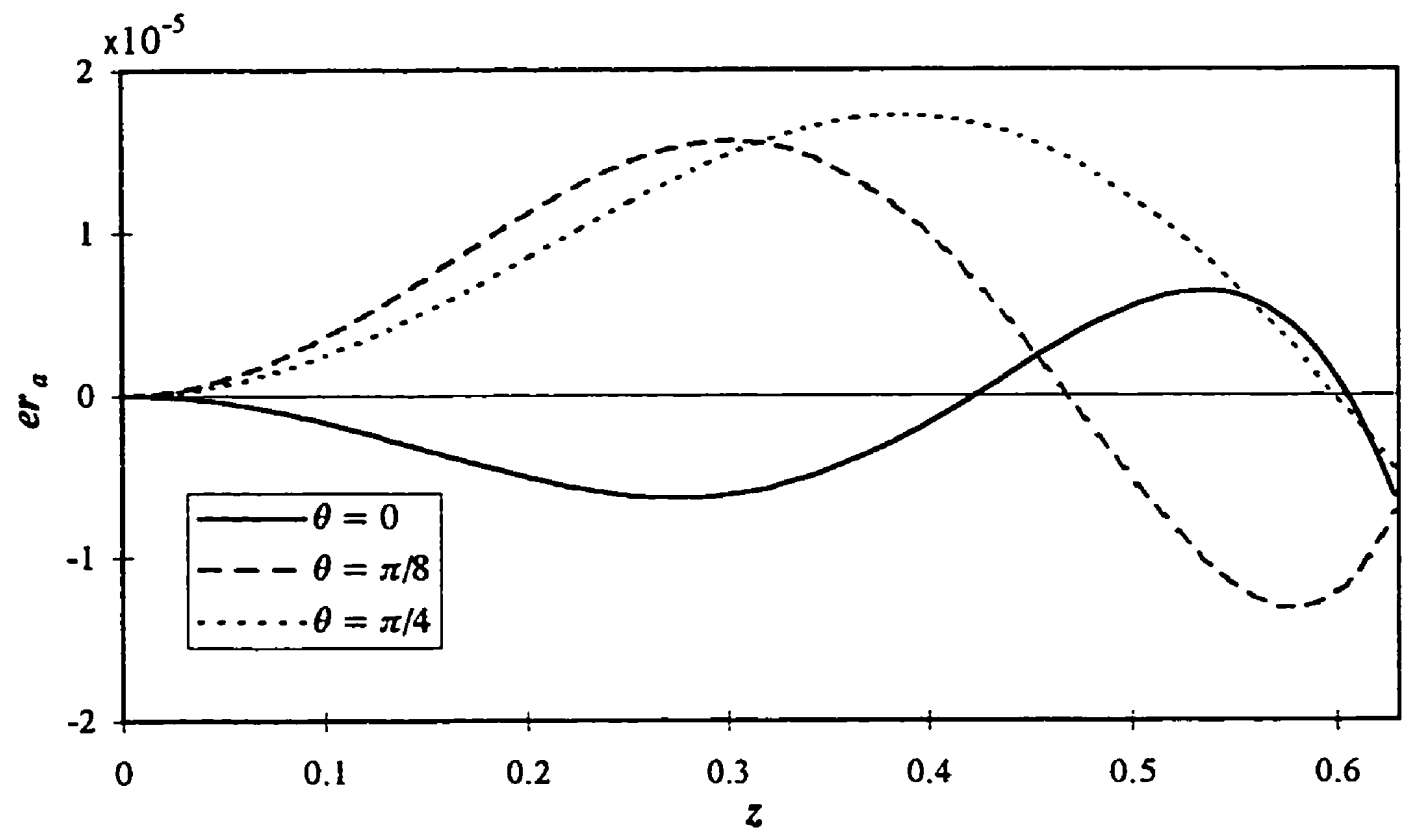

b)

Figure 12: Local amplitude errors for 010 scheme with different values of $\theta$ at a) $C=1$ and b) $C=1 / 2$.

associated with problems which have waves resolved by at least $10 \mathrm{PPW}$. The errors are bounded by $-2 \times 10^{-5}$ and $2 \times 10^{-5}$. The figures only show a finite number of combinations of the Courant number and the angle of incidence, but the errors remain bounded by $-2 \times 10^{-5}$ and 
$2 \times 10^{-5}$ for all $C \in(0,1], \theta \in[0,2 \pi]$, and $z \in[0, \pi / 3]$. Also, when extended to problems in three dimensions, using a regular Cartesian grid, the 010 scheme produces errors which are bounded by the above values when modeling a plane harmonic wave traveling in any direction.

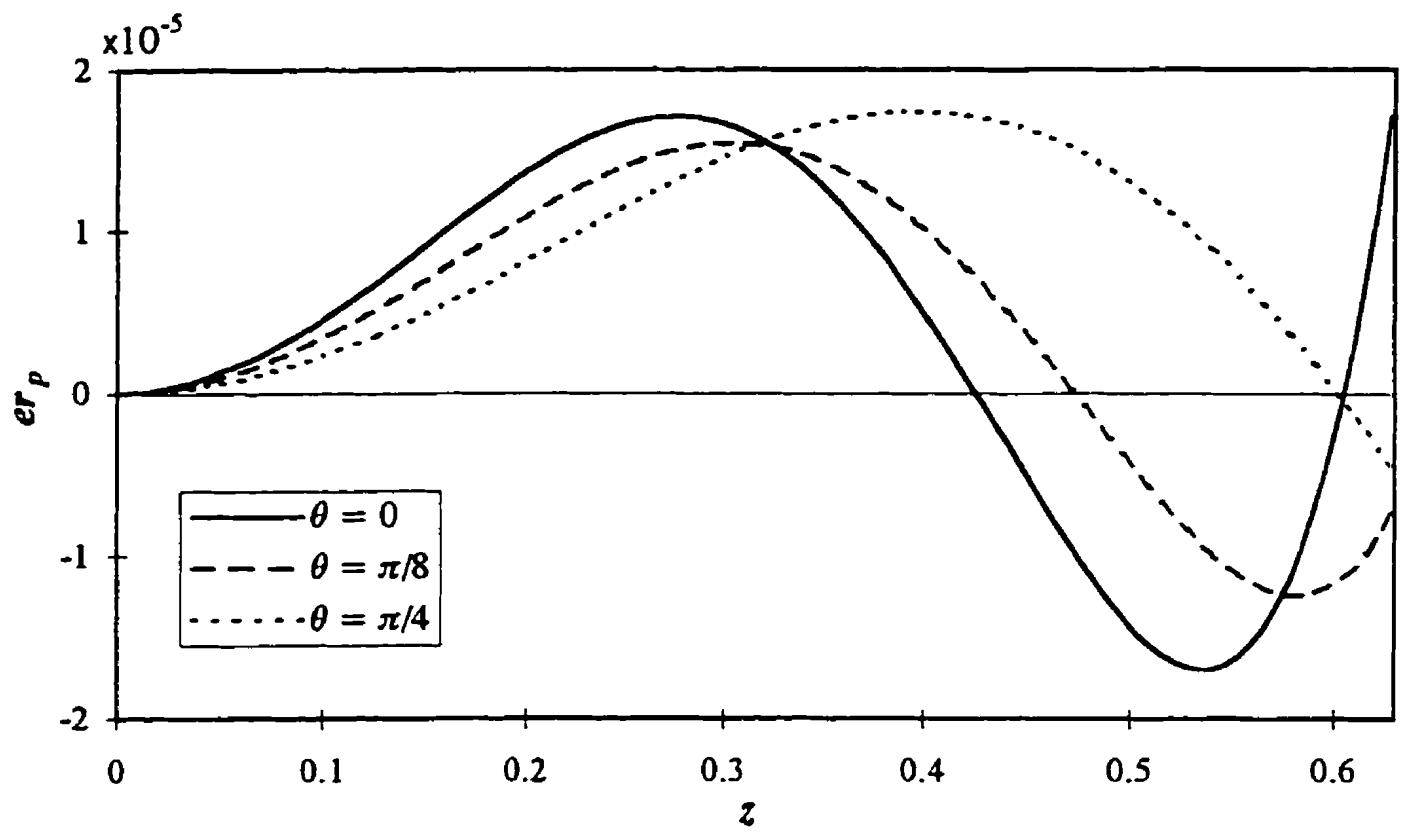

a)

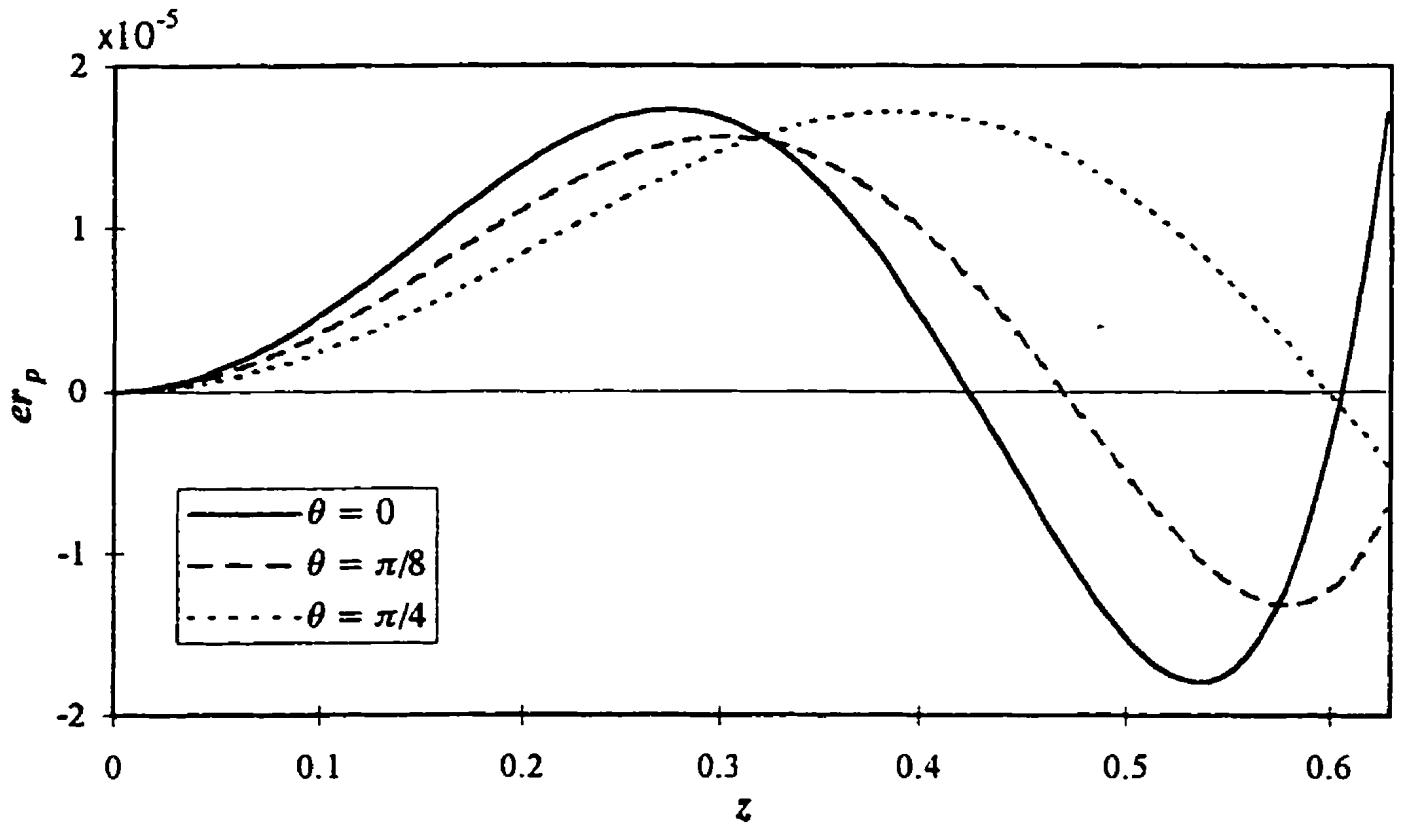

b)

Figure 13: Normalized local phase errors for $\mathrm{O} 10$ scheme with different values of $\theta$ at a) $C=1$ and b) $C=1 / 2$. 


\subsection{Numerical Boundary Schemes}

When simulating the propagation of electromagnetic waves through a dielectric material, the dielectric must be modeled using a numerical domain of finite extent. When the boundary of the numerical domain is approached, the interior schemes can no longer be used when their computational stencils extend out of the numerical domain as shown in Figure 3. For this reason numerical boundary schemes which have computational stencils that do not extend outside of the numerical domain are used.

Near a boundary, a locally one-dimensional characteristic formulation of the governing equations is used. The electromagnetic fields are split into a pair of waves, one which exits and one which enters the domain at an angle normal to the boundary. This is discussed fully in the next chapter. When calculating the spatial derivatives of these characteristic variables, upwind and upwind-biased finite-difference schemes are used. For the waves which are moving out of the domain, regular fifth-order one-sided and upwind-biased schemes are used. These schemes are developed by using the interior scheme up to the boundary, and using fifth-order polynomial extrapolation to obtain the necessary field values outside the domain. For example, if an interior operator with a seven-point computational stencil is used up to the first node in the domain, extrapolation is needed to find the field values at the $0^{\text {th }},-1^{\text {st }}$, and $-2^{\text {nd }}$ "nodes". Note that the location of these "nodes" must be known. The following are the extrapolation schemes which are used:

$$
\begin{gathered}
u_{0}=6 u_{1}-15 u_{2}+20 u_{3}-15 u_{4}+6 u_{5}-u_{6} \\
u_{-1}=21 u_{1}-70 u_{2}+105 u_{3}-84 u_{4}+35 u_{5}-6 u_{6} \\
u_{-2}=56 u_{1}-210 u_{2}+336 u_{3}-280 u_{4}+120 u_{5}-21 u_{6}
\end{gathered}
$$

When the interior scheme is used up to the last node in the domain, $i_{\max }$, the following extrapolation schemes must be used to find the field values at the $i_{\max }+1^{\text {st }}, i_{\max }+2^{\text {nd }}$, and $i_{\max }+3^{\text {rd }}$ "nodes":

$$
\begin{aligned}
& u_{i_{\max }+1}=6 u_{i_{\max }}-15 u_{i_{\max }-1}+20 u_{i_{\text {max }}-2}-15 u_{i_{\max }-3}+6 u_{i_{\max }}-4-u_{i_{\max }}-5 \\
& u_{i_{\max }+2}=21 u_{i}-70 u_{i_{\operatorname{man}}}+105 u_{i_{\max }-2}-84 u_{i_{\max }-3}+35 u_{i_{\max }-4}-6 u_{i_{\max }-5}
\end{aligned}
$$

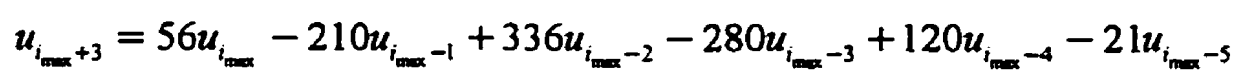


The use of regular one-sided upwind-biased fifth-order finite-difference operators will lead to numerical instabilities when calculating derivatives of the incoming characteristic variables. To find a stable and accurate inflow NBS, a two-parameter scheme, originally presented in [88], is used. When approximating the derivatives of the fields near the $0^{\text {th }}$ "node", at which incoming field values are used as boundary conditions, the NBS is given by

$$
\begin{array}{r}
\left(\delta_{x} u\right)_{1}=\frac{1}{\Delta x}\left[\begin{array}{r}
(\alpha-1 / 5) u_{0}-(6 \alpha+13 / 12) u_{1}+(15 \alpha+2) u_{2}-(20 \alpha+1) u_{3} \\
+(15 \alpha+1 / 3) u_{4}-(6 \alpha+1 / 20) u_{5}+\alpha u_{6}
\end{array}\right] \\
\left(\delta_{x} u\right)_{2}=\frac{1}{\Delta x}\left[\begin{array}{r}
(\beta+1 / 20) u_{0}-(6 \beta+1 / 2) u_{1}+(15 \beta-1 / 3) u_{2}-(20 \beta-1) u_{3} \\
+(15 \beta-1 / 4) u_{4}-(6 \beta-1 / 30) u_{5}+\beta u_{6}
\end{array}\right]
\end{array}
$$

The above NBS is at least fifth-order accurate and has a maximum accuracy which is sixthorder when $\alpha=1 / 30$ and $\beta=-1 / 60$. Setting $\alpha=3 / 20$ and $\beta=1 / 10$ gives the following fifth-order NBS which is used for the incoming waves:

$$
\begin{gathered}
\left(\delta_{x} u\right)_{1}=\frac{1}{60 \Delta x}\left[-3 u_{0}-119 u_{1}+255 u_{2}-240 u_{3}+155 u_{4}-57 u_{5}+9 u_{6}\right] \\
\left(\delta_{x} u\right)_{2}=\frac{1}{60 \Delta x}\left[9 u_{0}-66 u_{1}+70 u_{2}-60 u_{3}+75 u_{4}-34 u_{5}+6 u_{6}\right]
\end{gathered}
$$

The above NBS is a modified version of the one presented by Zingg et al. [89, 95], which was found to be unstable on some curvilinear grids. When approximating the derivatives of the fields near the $i_{\max }+1^{\text {t }}$ "node", the NBS is given by

$$
\begin{aligned}
& \left(\delta_{x} u\right)_{i_{\max }}=\frac{1}{60 \Delta x}\left[\begin{array}{r}
3 u_{i_{\max }+1}+119 u_{i_{\max }}-255 u_{i_{\max }-1}+240 u_{i_{\max }-2} \\
-155 u_{i_{\max }-3}+57 u_{i_{\max }}-4-9 u_{i_{\max }-5}
\end{array}\right] \\
& \left(\delta_{x} u\right)_{i_{\max }-1}=\frac{1}{60 \Delta x}\left[\begin{array}{r}
-9 u_{i_{\max }+1}+66 u_{i_{\max }}-70 u_{i_{\max }-1}+60 u_{i_{\max }-2} \\
-75 u_{i_{\max }-3}+34 u_{i_{\max }}-6
\end{array}\right]
\end{aligned}
$$

\subsection{Stability}

It can be shown, using a Fourier analysis, that the maximum-order scheme is stable up to $C \approx 1.5$ in one dimension, and the scheme optimized for 10 PPW is unconditionally unstable. Zingg et al. [95] show that the instability in the O10 scheme is very mild, and when 
coupled with the inflow NBS ( 3.56 ) and the outflow NBS, which uses the extrapolation schemes in ( 3.54 ), asymptotic, or time, stability is achieved on a finite one-dimensional numerical domain. The stability regions for the time-marching methods can be found in this reference, as well as plots of the eigenvalues associated with the spatial operators. It is also shown that the optimized scheme can be safely used for well over 30,000 time-steps, with a Courant number of one, before the mild instability will have any impact on the simulation. Running a simulation for 30,000 time-steps with $C=1$, and using a grid with 10 PPW resolution, is equivalent to propagating a wave 3,000 wavelengths. The $\mathrm{O} 10$ scheme is not designed to be used for such long simulations since the MO scheme is much more accurate for distances of travel greater than 300 wavelengths. The stability of both schemes was demonstrated in [91] where a number of simulations of one-dimensional electromagnetic scattering are presented.

The stability of the above methods has not been demonstrated analytically for problems in two dimensions where complex grids are used to join different material domains, and for general two-dimensional curvilinear grids. This is quite a formidable problem and further research must be performed before the numerical stability of the schemes can be fully determined for such complex geometries. The MO scheme and the O10 scheme have both been used to model many scattering problems in two dimensions, where a number of dielectric materials have been used, and where curvilinear grids are used (see, for example, the results in Chapter 5 and $[29,30])$. Useful engineering data has been obtained, and no numerical instabilities were evident, for simulations which lasted for thousands of time-steps. 


\section{Interface and Boundary Treatment}

When dealing with the scattering of electromagnetic waves, a numerical simulation must be equipped to handle an interface between two regions which have different material properties. The simulation must also allow for the truncation of the numerical domain far from the scatterer. A separate computational domain is used for each material, as shown in Figure 14. Except near material interfaces, each domain can be handled individually at each stage in the time-marching scheme. At an interface, information must be passed between domains in a way which preserves the physics of the problem. The values of the fields at the next stage in the time-marching scheme are calculated by assuming that the field values along all the interfaces are known during the current stage. Near an interface a special set of conditions are used which model the waves moving in and out of a domain. At the outer boundary of the problem (the outer boundary of ND $\mathbf{O}$ in the figure) waves must not produce any significant reflections as they pass out of the numerical domain. This is achieved by using special far-field boundary conditions.

\section{ND 0}

$$
\begin{aligned}
& \varepsilon_{0} \\
& \mu_{0}
\end{aligned}
$$

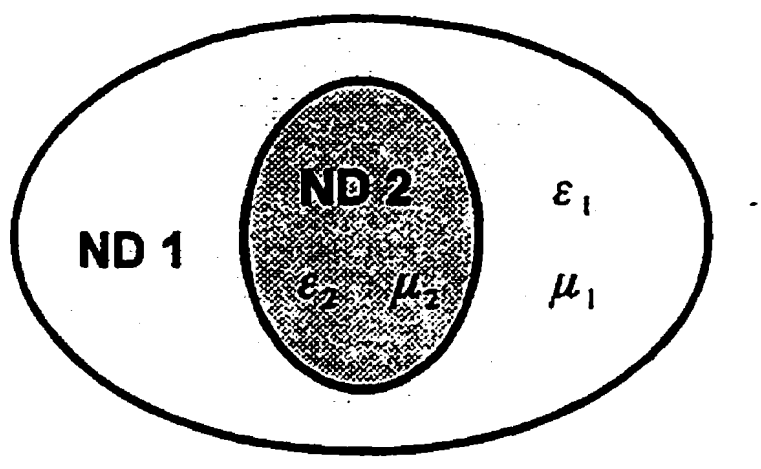

Figure 14: Alignment of Numerical Domains (ND) with Dielectric Materials 
Three separate boundary types are considered: an interface between two dielectric regions, the boundary between a dielectric and a perfect conductor, and the far-field boundary. At the interface between two dielectric media and at the surface of a perfect conductor, a locally one-dimensional characteristic formulation of the governing equations is used [24]. Using this approach, characteristic variables can be found which represent incoming and outgoing waves along lines normal to the interface. When calculating the spatial derivatives of these characteristic variables, upwind and upwind-biased finite-difference schemes, which are given in $\S 3.2$, are used.

\subsection{Dielectric Interfaces}

Figure 15 shows the different field values and material properties close to a general material interface. When the TM set of equations are used, there are six unknowns, $D_{z}^{(1)}, D_{z}^{(2)}$, $B_{x}{ }^{(1)}, B_{x}{ }^{(2)}, B_{y}{ }^{(1)}$, and $B_{y}{ }^{(2)}$, which must be stored at any point along the interface. Field values are stored on the interface for both Regions (1) and (2), which allows for possible discontinuities in the flux densities across the interface.

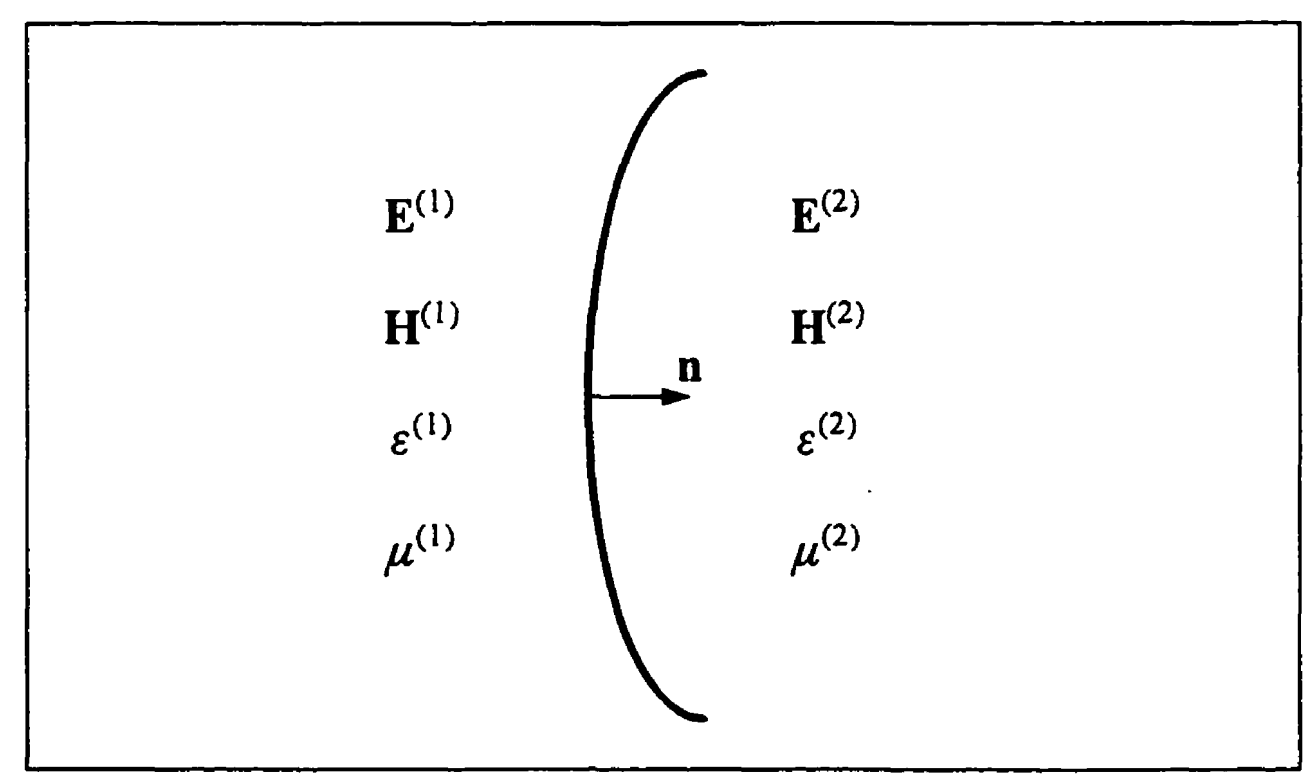

Figure 15: Field values and material properties near interface

This research is concerned with problems in which there is no current flowing along the interface, and no charge present at the interface. With these constraints, the physical boundary 
conditions given in $\S 2.5$ reduce to

$$
\begin{aligned}
& E_{l}^{(1)}=E_{l}^{(2)} \\
& H_{\imath}^{(1)}=H_{l}^{(2)} \\
& D_{n}^{(1)}=D_{n}^{(2)} \\
& B_{n}^{(1)}=B_{n}^{(2)}
\end{aligned}
$$

In the TM case, the electric field intensity and electric flux density vectors point out of the $x-y$ plane, thus ( 4.3 ) holds automatically and (4.1) simplifies to

$$
E_{z}^{(1)}=E_{z}^{(2)}
$$

\subsubsection{Curvilinear Coordinates}

Consider a body-fitted grid which ensures that the interface between different materials will lie on a $\xi=$ constant curve. Figure 16 shows an example of such a body-fitted coordinate

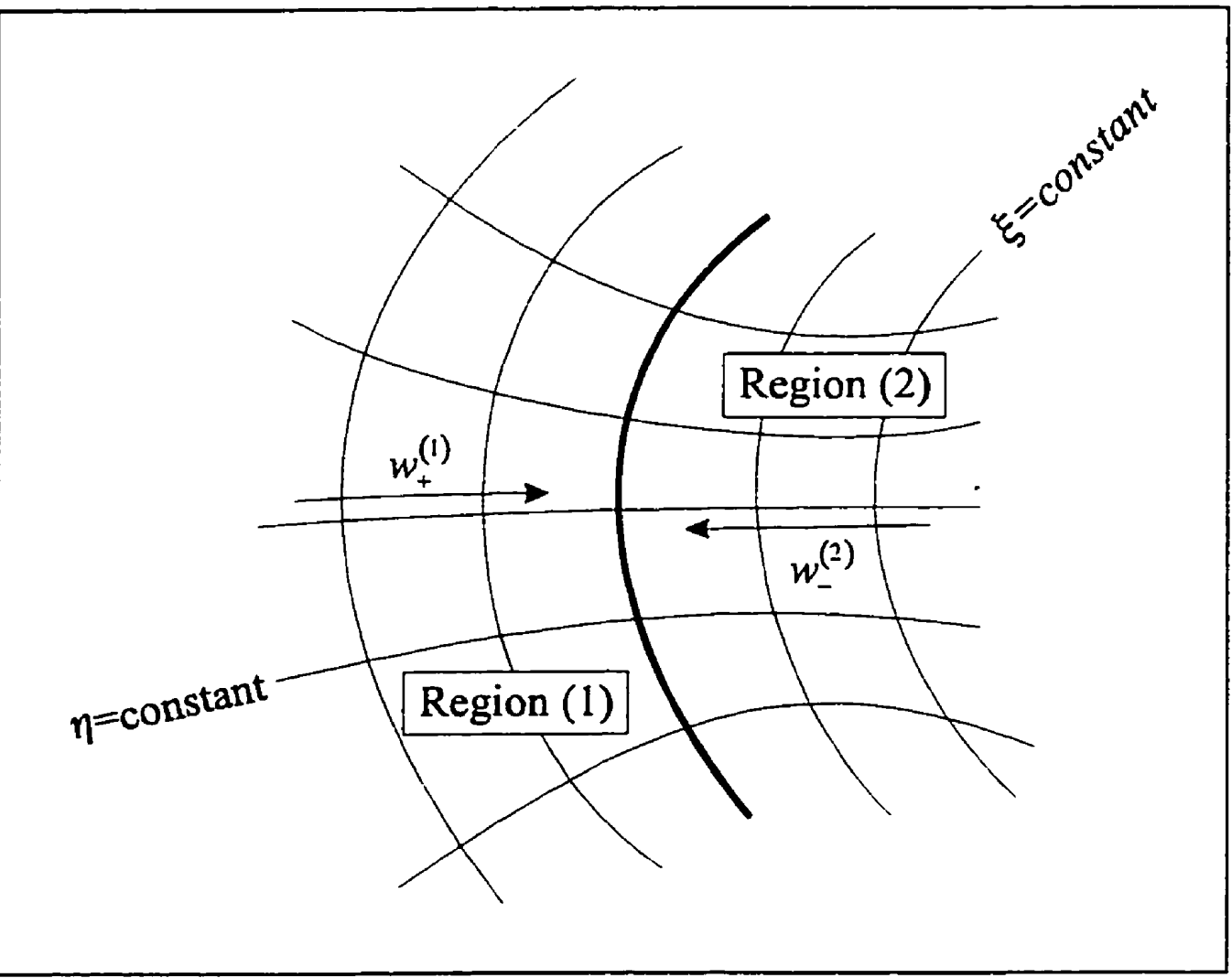

Figure 16: Example of a body fitted grid showing the characteristic variables associated with the outgoing waves 
system, where the positive $\xi$ direction points from Region (1) to Region (2). The fields are extrapolated to the interface from both materials and the characteristic variables associated with the outgoing waves are determined along the interface. These values are then coupled with the physical jump conditions, given above, in order to determine the values of the fields along both sides of the interface. The incoming characteristics can then be determined, using the interface field values as the boundary conditions, for each of the respective regions.

The field variables are extrapolated to the interface, along constant $\boldsymbol{\eta}$ lines, using the fifth-order polynomial methods given in equations ( 3.53 ) and (3.54). The characteristic variables were determined in $\S 2.4 .2$ and can be written as

$$
w_{\bullet}^{(1)}=\eta_{l}^{(1)} D_{z}^{(1)}+B_{l}^{(1)}
$$

and

$$
w_{-}^{(2)}=\eta_{t}^{(2)} D_{z}^{(2)}-B_{t}^{(2)}
$$

Note that

$$
B_{t}=\frac{\frac{\partial \xi}{\partial y} B_{x}-\frac{\partial \xi}{\partial x} B_{y}}{\left[\left(\frac{\partial \xi}{\partial x}\right)^{2}+\left(\frac{\partial \xi}{\partial y}\right)^{2}\right]^{1 / 2}}
$$

is the component of $\mathbf{B}$ tangent to a $\xi=$ constant curve, and

$$
B_{n}=\frac{\frac{\partial \xi}{\partial x} B_{x}+\frac{\partial \xi}{\partial y} B_{y}}{\left[\left(\frac{\partial \xi}{\partial x}\right)^{2}+\left(\frac{\partial \xi}{\partial y}\right)^{2}\right]^{1 / 2}}
$$

is the component of $\mathbf{B}$ normal to a $\xi=$ constant curve. This gives two equations for the unknown values $D_{z}^{(1)}, D_{z}^{(2)}, B_{t}^{(1)}$, and $B_{t}^{(2)}$. The characteristic variables depend on the orientation of the local $(\xi, \eta)$ coordinate systems at the interface. For the example above, the characteristic variable representing a wave moving in the positive $\xi$ direction is obtained along the Region (1) side of the interface, and the characteristic variable representing a wave moving in the negative $\xi$ direction is obtained along the Region (2) side of the interface. If, for example, the local coordinate systems were arranged such that one approaches the interface from 
Region (1) along a line of constant $\xi$ with $\eta$ decreasing, and approaches the interface from Region (2) along a line of constant $\eta$ with $\xi$ increasing, then different quantities would be needed. For this case, the characteristic variable representing a wave moving in the negative $\eta$ direction is used on the Region (1) side, and the characteristic variable representing a wave moving in the positive $\xi$ direction is used on the Region (2) side. Care must be taken when defining the components of the fields normal and tangential to the interface, as they will also be dependent on the orientation of the local $(\xi, \eta)$ coordinate systems.

Two more equations are needed to solve for the four unknowns given above. These equations can be found by using the interface jump conditions, ( 4.2 ) and ( 4.5 ), which can be written as

$$
\begin{aligned}
& \varepsilon^{(2)} D_{z}^{(1)}-\varepsilon^{(1)} D_{z}^{(2)}=0 \\
& \mu^{(2)} B_{l}^{(1)}-\mu^{(1)} B_{l}^{(2)}=0
\end{aligned}
$$

The four relationships given above, (4.6), (4.7), (4.10), and (4.11), can be used to determine the following field values on the interface:

$$
\begin{gathered}
D_{z}^{(1)}=\frac{w_{+}^{(1)}+\frac{\mu^{(1)}}{\mu^{(2)}} w_{-}^{(2)}}{\eta_{l}^{(1)}\left(1+\frac{\eta_{l}^{(1)}}{\eta_{t}^{(2)}}\right)} \\
D_{z}^{(2)}=\frac{\varepsilon^{(2)}}{\varepsilon^{(1)}} D_{z}^{(1)} \\
B_{t}^{(1)}=\frac{\eta_{t}^{(1)} w_{+}^{(1)}-\mu^{(1)} c^{(2)} w_{-}^{(2)}}{\eta_{t}^{(1)}+\eta_{t}^{(2)}} \\
B_{t}^{(2)}=\frac{\mu^{(2)}}{\mu^{(1)}} B_{t}^{(1)}
\end{gathered}
$$

The normal component of the magnetic flux density is single valued on the interface, as shown by (4.4). It is obtained by using the extrapolated field values to calculate $B_{n}$ along both sides of the interface, and then averaging these values. The values of $B_{x}$ and $B_{y}$ can now be determined from $B_{n}$ and $B_{t}$, using ( 4.8 ) and (4.9), along both sides of the interface. 


\subsubsection{Cartesian Coordinates}

Handling a dielectric interface in Cartesian coordinates is quite simple. The method can easily be obtained from the generalized curvilinear coordinate case given above. For example, when the interface lies along a line of constant $x$, as shown in Figure 17, the necessary relationships can be found by letting $\xi=x$ and $\eta=y$ in the equations given in the above section. It is clear from ( 4.8$)$ and (4.9) that $B_{t}=-B_{y}$ and $B_{n}=B_{x}$. The field values on an interface which lies along a line of constant $y$ value can be found in a similar manner by letting $\xi=y$ and $\eta=-x$.

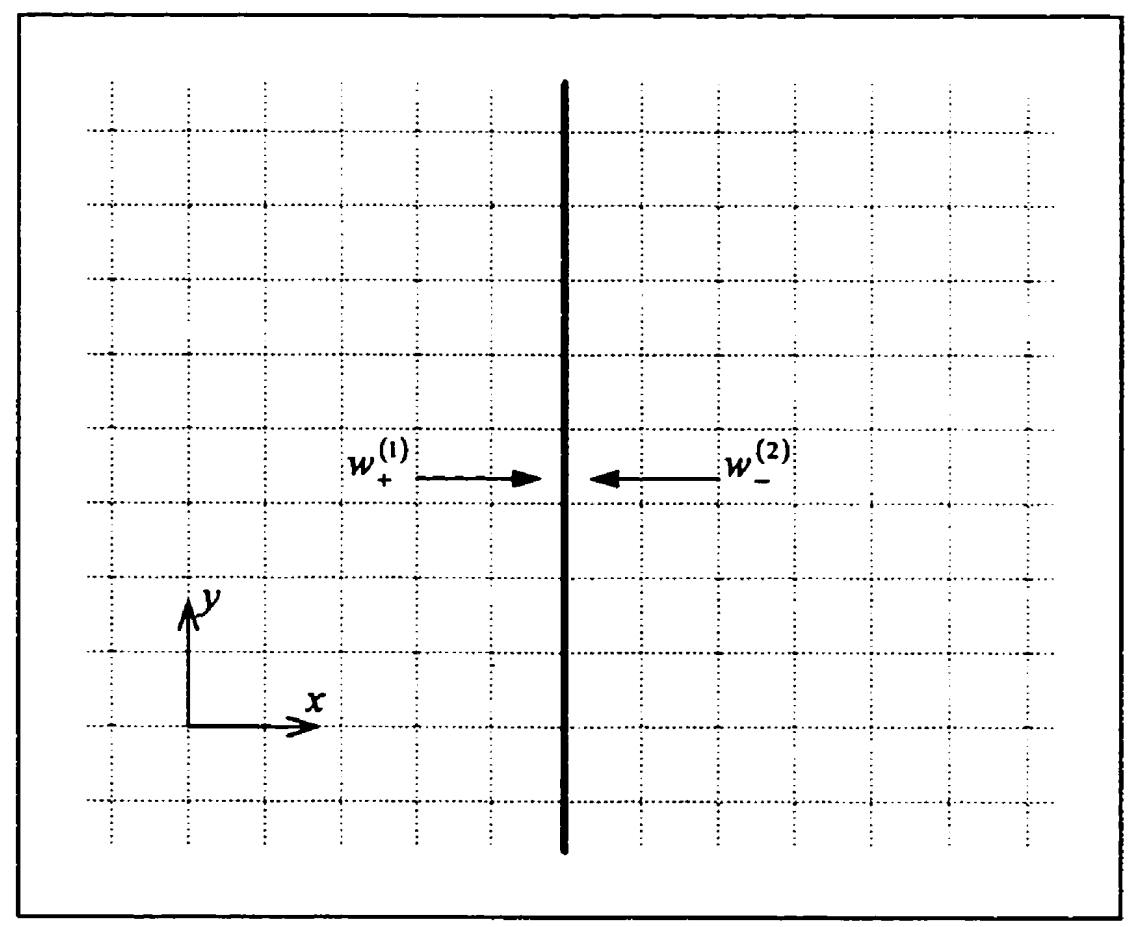

Figure 17: Example of Cartesian grid with interface along line of constant $x$

The following are the characteristic variables which are needed on both sides of the interface:

$$
\begin{aligned}
& w_{+}^{(1)}=\eta_{I}^{(1)} D_{z}^{(1)}-B_{y}^{(1)} \\
& w_{-}^{(2)}=\eta_{I}^{(2)} D_{z}^{(2)}+B_{y}^{(2)}
\end{aligned}
$$

The above equations give two constraints for the four unknown field values, $D_{z}^{(1)}, D_{z}^{(2)}$, $B_{y}{ }^{(1)}$, and $B_{y}{ }^{(2)}$. The interface jump conditions, (4.10) and (4.11), give two more constraints. For this case, equation ( 4.11 ) can be written as: 


$$
\mu^{(2)} B_{y}^{(1)}-\mu^{(1)} B_{y}^{(2)}=0
$$

Solving these four equations results in the following values:

$$
\begin{gathered}
D_{z}^{(1)}=\frac{w_{+}^{(1)}+\frac{\mu^{(1)}}{\mu^{(2)}} w_{-}^{(2)}}{\eta_{l}^{(1)}\left(1+\frac{\eta_{I}^{(1)}}{\eta_{l}^{(2)}}\right)} \\
D_{z}^{(2)}=\frac{\varepsilon^{(2)}}{\varepsilon^{(1)}} D_{z}^{(1)} \\
B_{y}^{(1)}=\frac{\mu^{(1)} c^{(2)} w_{-}^{(2)}-\eta_{I}^{(1)} w_{+}^{(1)}}{\eta_{l}^{(1)}+\eta_{l}^{(2)}} \\
B_{y}^{(2)}=\frac{\mu^{(2)}}{\mu^{(1)}} B_{y}^{(1)}
\end{gathered}
$$

The $x$ component of the magnetic flux density is single valued on the interface and is obtained by determining the average value of $B_{x}$ from the extrapolated fields.

\subsection{Perfect Conductors}

When modeling a scattering problem where some of the materials being considered can be treated as perfect conductors, the correct boundary conditions must be used along an interface between the conductor and a dielectric material. Because an electromagnetic wave does not propagate into the interior of a perfect conductor, a grid does not need to be used in any domain which represents perfectly conducting material. The boundary conditions on the interface were given in $\S 2.5$, and are written here, for the TM set of equations, as:

$$
\begin{aligned}
& E_{t}=0 \\
& H_{t}=J_{s} \\
& D_{n}=0 \\
& B_{n}=0
\end{aligned}
$$

where $J_{s}$ is the surface current present on the interface. Figure 18 shows an example of a grid near a perfect conductor whose surface lies along a line of constant $\xi$. 


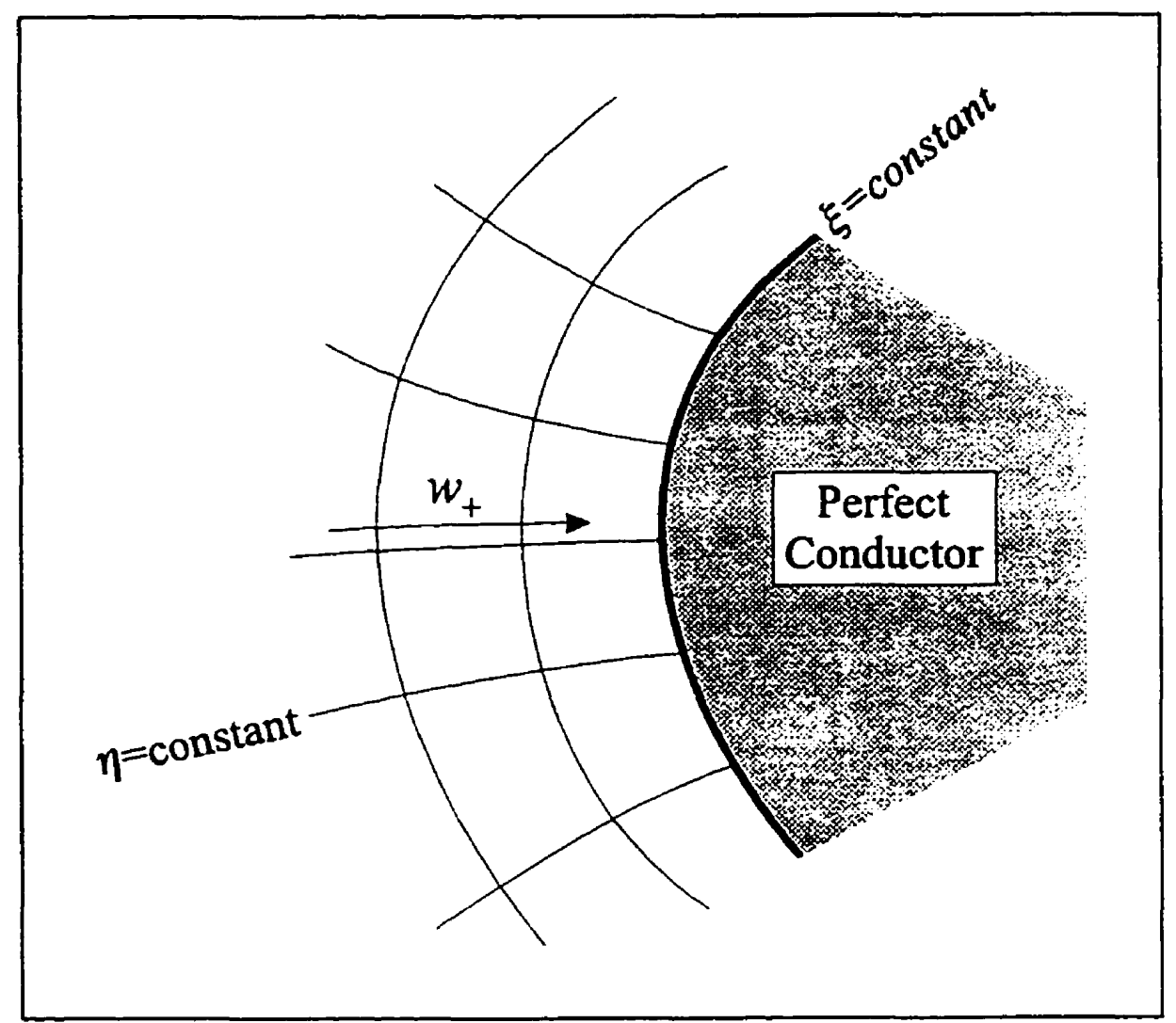

Figure 18: Example of a body fitted grid near the surface of a perfect conductor

For the TM set of Maxwell's equations ( 4.25 ) is automatically satisfied since the electric flux density points out of the plane, and ( 4.23 ) sets its magnitude to zero, i.e., $D_{z}=0$ on the surface of the perfect conductor. The tangential component of the magnetic flux density, and indirectly the surface current, is found by extrapolating the fields to the conductor's surface, and then calculating the outgoing characteristic variable. For example, if the surface of the perfect conductor lies along a line of constant $\xi$, with $\xi$ increasing as the conductor is approached, then the fields are extrapolated to the surface along a line of constant $\eta$ using a fifth-order polynomial method, and $w_{+}$is determined. Since $D_{z}=0,(4.6)$ gives

$$
B_{t}=w_{+}
$$

where $B_{\mathrm{t}}$ is defined by (4.8). The Cartesian components of the magnetic flux density are determined using this value, and the fact that $B_{n}=0$.

If a Cartesian grid is being used and the surface of the perfect conductor lies along a line of constant $x$, with $x$ increasing as one approaches the conductor, then the proper boundary conditions are found by replacing $B_{n}$ with $B_{x}$ and $B_{t}$ with $-B_{y}$. This results in $D_{z}=B_{x}=0$ and 
$B_{y}=-w_{+}$along the surface of the perfect conductor.

\subsection{Far-Field Boundaries}

To model the problem of waves moving out into free space the numerical domain must be artificially truncated. The NBS used at this outer boundary is often a large source of error due to spurious reflections which occur when the scattered waves exit the numerical domain. There are a number of methods discussed in the literature for dealing with this problem $[2,3$, $12,16,22,75]$. The simulations discussed in the next chapter use two different types of farfield boundary conditions (FFBC), the first is based upon a radiation condition given by Tam and Webb [73], and the second is based on a locally one-dimensional characteristic splitting.

\subsubsection{Radiation FFBC}

A modified version of the radiating boundary condition introduced by Tam and Webb, which was found to be the best method of those tested by Hixon et al. [25], is used. This method truncates the domain by using a far-field radiation condition which only allows disturbances to propagate in the outward direction. The method was formulated for the governing equations of computational aeroacoustics, but it can be applied directly to electromagnetic problems. In two dimensions, when the free-stream velocity is set to zero, the governing equations of aeroacoustics have a one-to-one correspondence with the TM set of Maxwell's equations. The electromagnetic fields are related to the aeroacoustic variables as follows

$$
D_{z}=\rho \quad B_{x}=v \quad B_{y}=-u
$$

The condition is applied by enforcing the following partial differential equation around the outer boundary of the domain:

$$
\left(\frac{1}{c} \frac{\partial}{\partial t}+\frac{\partial}{\partial r}+\frac{a}{2 r}\right)\left[\begin{array}{l}
D_{z} \\
B_{x} \\
B_{y}
\end{array}\right]=0
$$

where $r=\sqrt{x^{2}+y^{2}}$. The origin should be near the center of the numerical domain. When $a=1$ the operator given in [73] is recovered which is the asymptotic solution for an electromagnetic wave propagating away from the origin as $r \rightarrow \infty$, and it is $\mathrm{O}\left(r^{-5 / 2}\right)$. Using the operator with this value of $a$ results in the numerical schemes becoming unstable for some 
curvilinear grids. This instability is present even when second-order spatial finite-difference schemes are used. By setting $a=3$ a stable and accurate method results for all the schemes considered. A better understanding of the effects of changing the value of $a$ can be obtained by looking at the solution of the above equation, which is given by

$$
Q(r, t)=Q_{0} r^{-a / 2} e^{-j \omega(r / a t)}
$$

where $Q$ represents any of the unknown field values. This solution represents an outward traveling wave which is attenuated spatially by a factor of $r^{-a / 2}$. When $a=1$ the classical farfield attenuation of an outgoing cylindrical wave, $1 / \sqrt{r}$, is obtained (see, for example, [19]). When $a=3$ the wave will be attenuated at an increased rate as it moves away from the origin, which results in a stabilizing effect on the FFBC. Very good results have been obtained in a number of numerical simulations using this modified version of the original radiation FFBC, as is shown in the next chapter.

To apply (4.29), Tam and Webb use their seven-point operator out to the edge of the numerical domain. This necessitates the use of a number of points, which they call ghostpoints, that lie outside the domain. At these points the radiation boundary condition is enforced using appropriate one-sided operators as necessary. Figure 19 shows the ghost-points introduced near a corner of a grid, and examples of some computational stencils used by the interior and radiation operators. The simulations discussed in this work use a similar method. The spatial derivatives in the electromagnetic equations are handled out to the ghost-points using the seven point operators given by (3.1) and (3.2). The spatial derivatives in the radiation FFBC are handled in the ghost point region using the above mentioned interior operators and the one-sided outflow operators given in $\S 3.2$. The same time-marching method is used for both the electromagnetic equations and the radiation FFBC.

For an electromagnetic wave to propagate into the numerical domain, the method used in [73] must be modified since it only considers disturbances exiting the domain. To allow the incident field to enter the numerical domain, the domain is split into two regions, one which contains the total electromagnetic field, and one which contains only the scattered field (see, for example, [72]). The scattered field is stored on the ghost-points, and the total field is stored at all other nodes. To approximate the spatial derivatives in Maxwell's equations where 


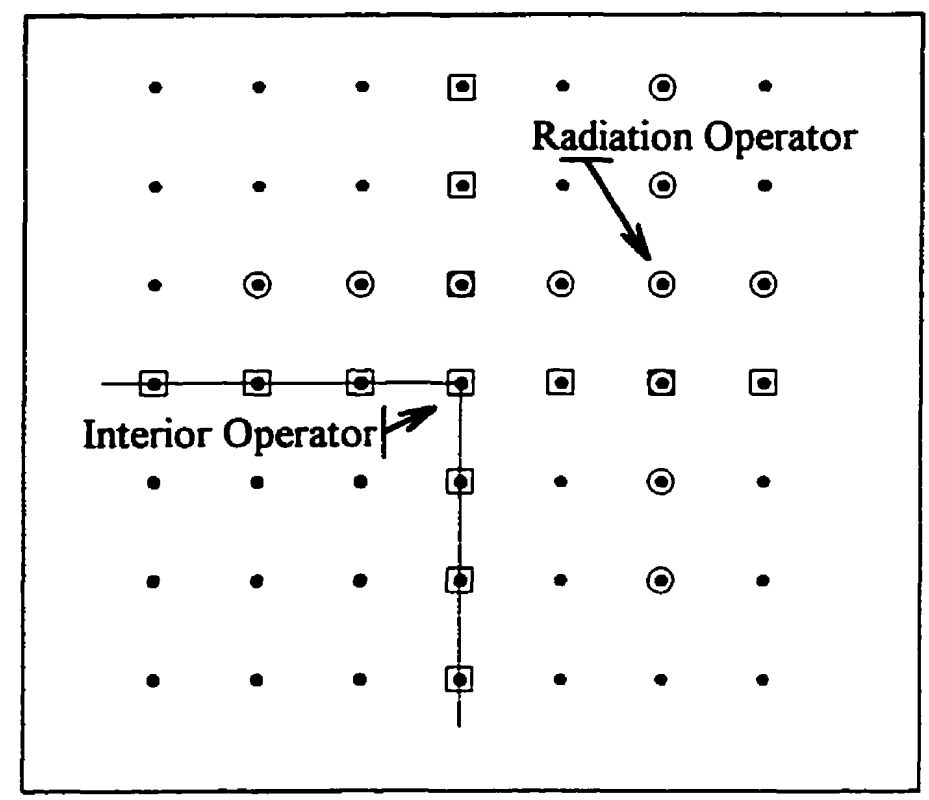

Figure 19: Example of stencils used near corner of numerical domain

the computational stencil will overlap the ghost-point region, the incident field is added to the scattered field at the necessary nodes in this ghost-point region. When calculating the spatial derivatives for the radiation condition, the incident field is subtracted from the total field at the necessary nodes in the interior domain. The incident field is a known function of space and time, and is known at every node during a simulation. Figure 20 shows an example of the

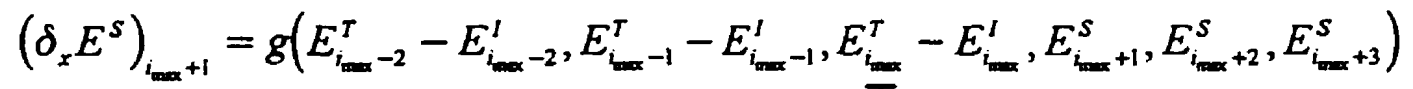

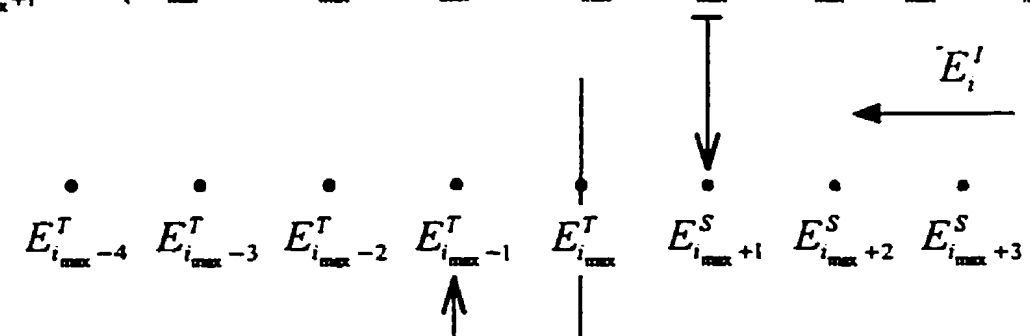

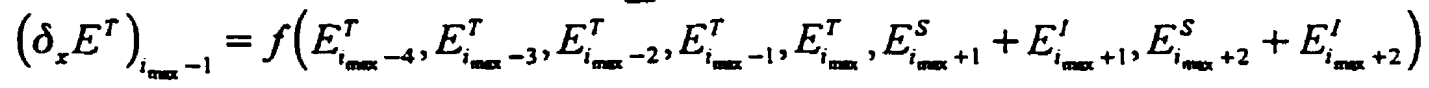

Figure 20: Fields stored near outer boundary of domain 
fields stored at the nodes near an outer boundary, as well as the data used when calculating the spatial derivatives of the fields in each domain.

\subsubsection{Characteristic FFBC}

The method based on a locally one-dimensional characteristic splitting is discussed in [29]. The characteristic form of Maxwell's equations, given in $\$ 2.4$, are used in the vicinity of the outer boundary, i.e., at any node in which the application of the interior operator will necessitate the use of ghost-points. When calculating the spatial derivatives of the outgoing characteristic variables, the NBS based on fifth-order polynomial extrapolation is used. To calculate the derivative of the incoming characteristic variables the regular seven-point interior operator is used. The incident field is used to calculate the incoming characteristics found at the ghost-points outside the domain, avoiding the use of the NBSs given in ( 3.56 ) and (3.57). Setting the incoming characteristic variables for the total field to the values associated with the incident field is the same as setting the incoming characteristic variables for the scattered field to zero. This method requires a simpler storage arrangement than the radiation FFBC because the total field can be used at every node in the domain, and no storage has to be used for the ghost-points since the incident field is a known quantity.

It has been shown in the literature (see, for example, [25]) that the use of this type of characteristic FFBC results in significant errors due to spurious refiections. This is illustrated in the next chapter by a number of tests which compare the accuracy of this method with the radiation condition discussed above. This method is useful for problems in which the scattered fields will not interact with the outer boundaries during the simulation. 


\section{Results and Discussion}

This chapter presents a number of numerical simulations which display the flexibility of the numerical methods given in previous chapters. The accuracy and efficiency of the methods are shown by performing grid resolution studies for several simulations, and comparing the results with those obtained using second- and fourth-order methods ( $\mathrm{C} 2$ and $\mathrm{C} 4$, respectively). The tests are concerned with the implementation of the high-order methods, MO and O10, for problems in two dimensions. An extensive study of the use of the high-order methods for the simulation of the one-dimensional Maxwell's equations can be found in [91]. Both regular Cartesian grids and curvilinear grids are used. The simulations test the formulation of the interface and boundary conditions by modeling dielectric and perfectly conducting scatterers, as well as determining the impact of the far-field boundary conditions.

The C2, C4, MO, and 010 numerical methods are all discussed extensively in Chapter 3. To calculate the spatial derivatives, the $\mathrm{C} 2$ method uses a second-order central-difference operator with a third-order filter while the $\mathrm{C} 4$ method uses a fourth-order central-difference operator with a third-order filter. Both C2 and C4 use the classical fourth-order RungeKutta time-marching scheme. The MO method uses a sixth-order central difference operator coupled with a fifth-order filter to calculate the spatial derivatives. A low storage six-stage Runge-Kutta scheme is used for the time-marching. The O10 method is similar to the MO method, the difference being that the coefficients of its finite-difference schemes are optimized for propagating waves roughly 300 wavelengths of travel. The high-order NBSs described in Chapter 3 are used with the MO and 010 methods. Regular third-order upwind and upwind biased NBSs, as well as third-order polynomial interpolation and extrapolation schemes, are used with the $\mathrm{C} 4$ method near the interfaces and boundaries. The $\mathrm{C} 2$ method uses a first-order upwind NBS, as well as first-order polynomial interpolation and extrapolation schemes, when required.

\subsection{Dielectric Square}

The first test simulates the scattering of a pulsed plane wave off of a dielectric square. Figure 21 shows an example grid as well as the geometry of the problem. The numerical 


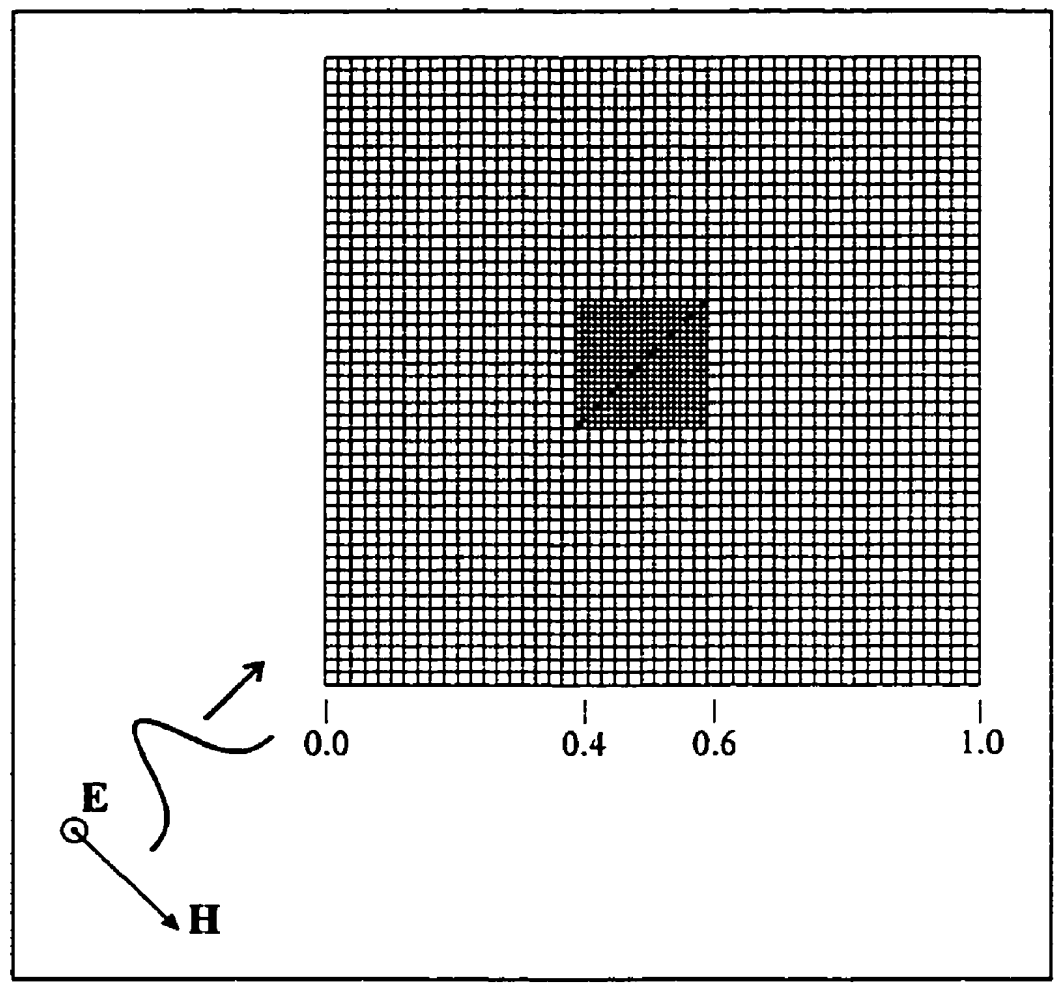

Figure 21: Example grid showing pulsed plane wave with Gaussian cross-section incident at $45^{\circ}$ on a dielectric square

domain is square, with $x$ and $y$ ranging from 0 to 1 , and the dielectric is located in the center of this region, at $0.4 \leq x \leq 0.6$ and $0.4 \leq y \leq 0.6$. The permittivity of the dielectric is four times the permittivity of the free space region, while the permeability is held constant. The wave speed in free space is normalized to one which results in a wave speed of a half in the dielectric. The domain is discretized using a Cartesian grid in which $\Delta x=\Delta y$. Two grids are used to model the problem, one for the domain outside the dielectric, and one for the interior of the dielectric. The grid density in the interior of the dielectric is twice that of free space. This ensures that the spatial resolution inside the dielectric is matched with that found in free space, since the width of the pulse will narrow as it slows down. A description of all the grids used in the tests can be found in Table 3. For all test cases, the time step is chosen to give a Courant number of one in free space. This results in a value of $\Delta t$ equal to the $\Delta x$ value of the free space domain. As a result of both the wave speed and the grid spacing inside the dielectric being one half the values found outside the dielectric, the Courant number will also have a 
value of one inside the dielectric. In all the simulations, the far-field boundary condition based on the characteristic splitting is used. Spurious reflections are not a problem because the incident field is imposed along the entire outer boundary, and the simulation is not run long enough for the reflected pulse to reach the outer boundary. Results are obtained for a Gaussian pulse incident upon the dielectric square at an angle of $45^{\circ}$. The incident electric field is given by

$$
E_{z}(x, y, t)=\exp \left[-\frac{1}{2 \sigma^{2}}\left(x \cos \frac{\pi}{4}+y \sin \frac{\pi}{4}+\frac{1}{2}-t\right)^{2}\right]
$$

with $\sigma=0.03$. The components of the incident magnetic fields can be calculated directly from this using Maxwell's equations and the angle of incidence.

\begin{tabular}{|c|c|c|}
\hline Total Nodes & $\begin{array}{c}\text { Resolution } \\
\text { Outside Dielectric }\end{array}$ & $\begin{array}{c}\text { Resolution } \\
\text { Inside Dielectric }\end{array}$ \\
\hline 2,800 & $50 \times 50$ & $20 \times 20$ \\
\hline 11,200 & $100 \times 100$ & $40 \times 40$ \\
\hline 17,500 & $125 \times 125$ & $50 \times 50$ \\
\hline 28,672 & $160 \times 160$ & $64 \times 64$ \\
\hline 44,800 & $200 \times 200$ & $80 \times 80$ \\
\hline 179,200 & $400 \times 400$ & $160 \times 160$ \\
\hline
\end{tabular}

Table 3: Grids used for dielectric square tests

For this simulation, the interface between the different materials lies along lines in the Cartesian grid. The jump conditions discussed in $\$ 4.1 .2$ can be used to obtain the field values along the interface. Since the grid in the interior of the dielectric square has twice the resolution of the grid outside the dielectric square, some of the values needed to obtain the fields on the interface must be interpolated. Also, because of the difficulty in defining tangential components of the fields, the corners must be handled as special cases. These two issues are discussed in detail in Appendix D. Furthermore, the corners produce singular fields. This is not taken into account in the present procedure, but the known singular behavior could be incorporated into the interpolation.

Contour plots of the electric field intensity, calculated at $t=1.4$, are shown for four different cases in Figure 22. Contours which represent negative values of the electric field are 


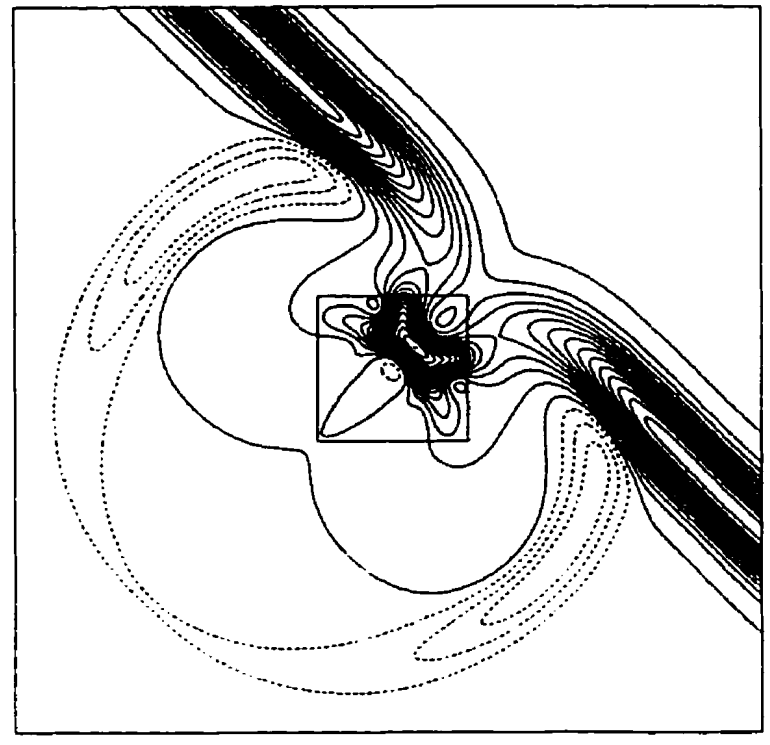

a)

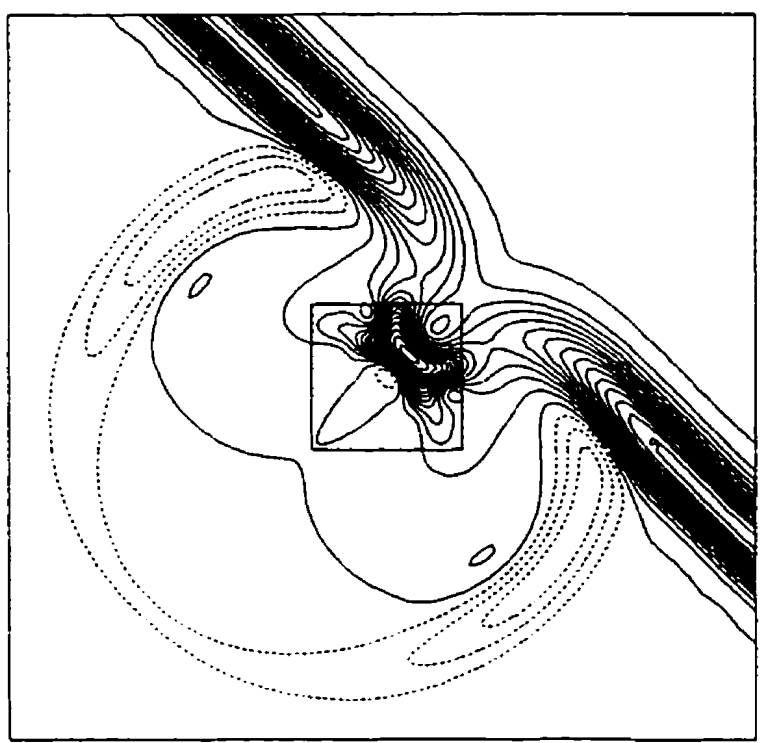

c)

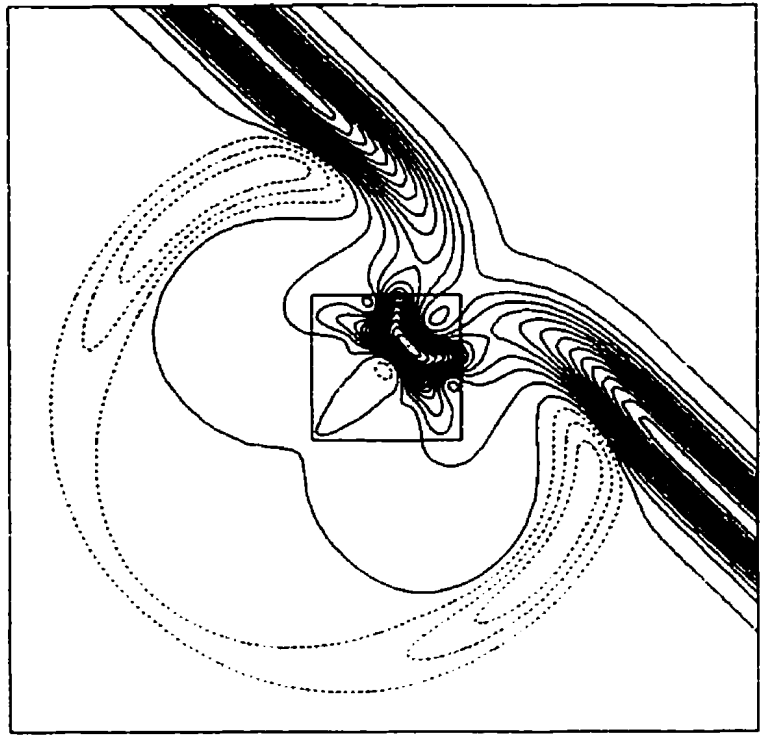

b)

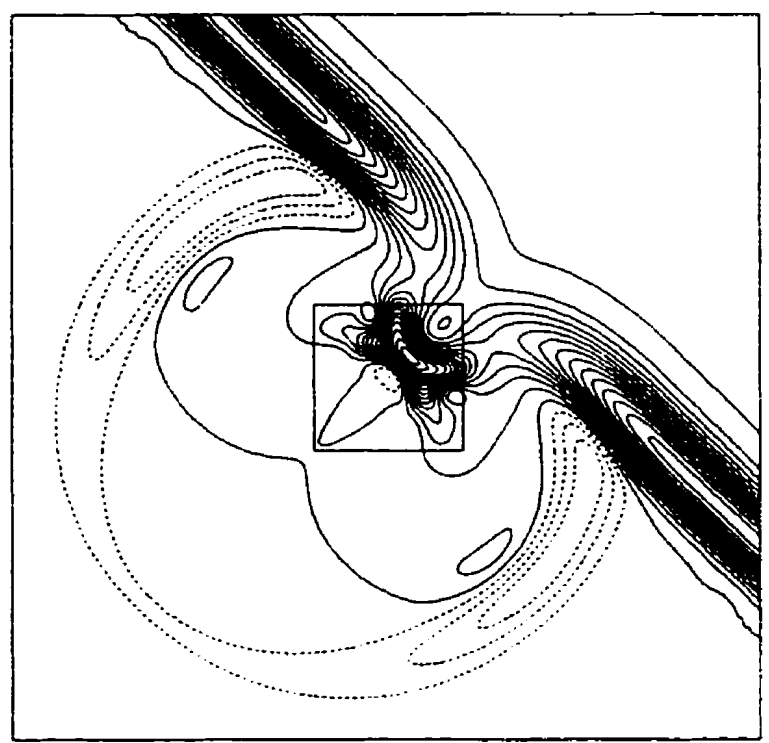

d)

Figure 22: Contour plots of electric field intensity for a) reference solution, b) MO using a grid with 11,200 nodes, c) C4 with 11,200 nodes, and d) C2 with 44,800 nodes

represented using dashed curves. Figure 22 a) shows a reference solution which is calculated using the MO scheme on a grid with 179,200 nodes. The results computed using the MO scheme on a coarser grid with 11,200 nodes is shown in Figure 22 b). The solution obtained using this coarser grid is virtually indistinguishable from the reference solution. Plots of the solutions obtained using C4 on the grid with 11,200 nodes and C2 on a grid with 44,800 
nodes are shown in Figures 22 c) and d), respectively. The C4 results show some small deviations from the reference solution, and even larger deviations are evident in the $\mathrm{C} 2$ results which are obtained on a grid with four times as many nodes.

The $z$ component of the electric field intensity along the diagonal of the dielectric square is shown in Figure 23 for the reference solution, the MO scheme on a grid with 11,200 nodes, the C4 scheme on a grid with 11,200 nodes, and the $C 2$ scheme on a grid with 44,800 nodes. The results obtained using the MO scheme lie very close to the reference solution. The C4 results are also fairly accurate, but there are some discernible deviations from the reference solution in the range $0.48<x(=y)<0.58$. The solution obtained by using the $\mathrm{C} 2$ method deviates considerably from the reference values.

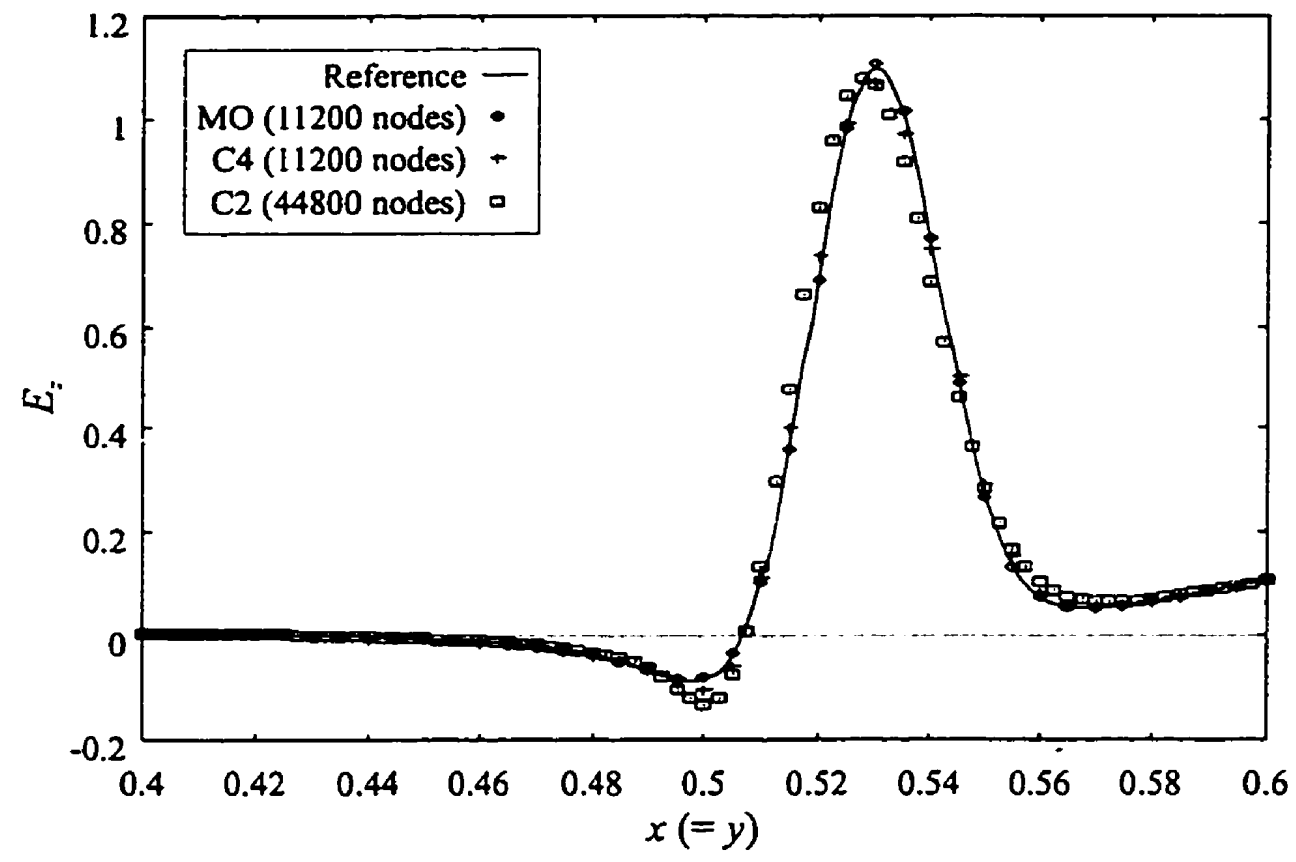

Figure 23: Electric field intensity across diagonal of dielectric square

Figure 24 shows the $\mathrm{L}_{2}$ norm of the errors in the $z$ component of the electric field intensity versus the total number of nodes in the grid. The error is normalized by the number of nodes in the numerical domain to allow the comparison of the results obtained using different grids. The plot shows the errors obtained by using the $\mathrm{C2}, \mathrm{C} 4, \mathrm{MO}$, and $\mathrm{O} 10$ schemes on a number of different grids. The errors are determined by considering the results obtained by a simulation which uses the MO scheme on the highly resolved grid as an accurate reference solution. The following formula is used to calculate the error: 


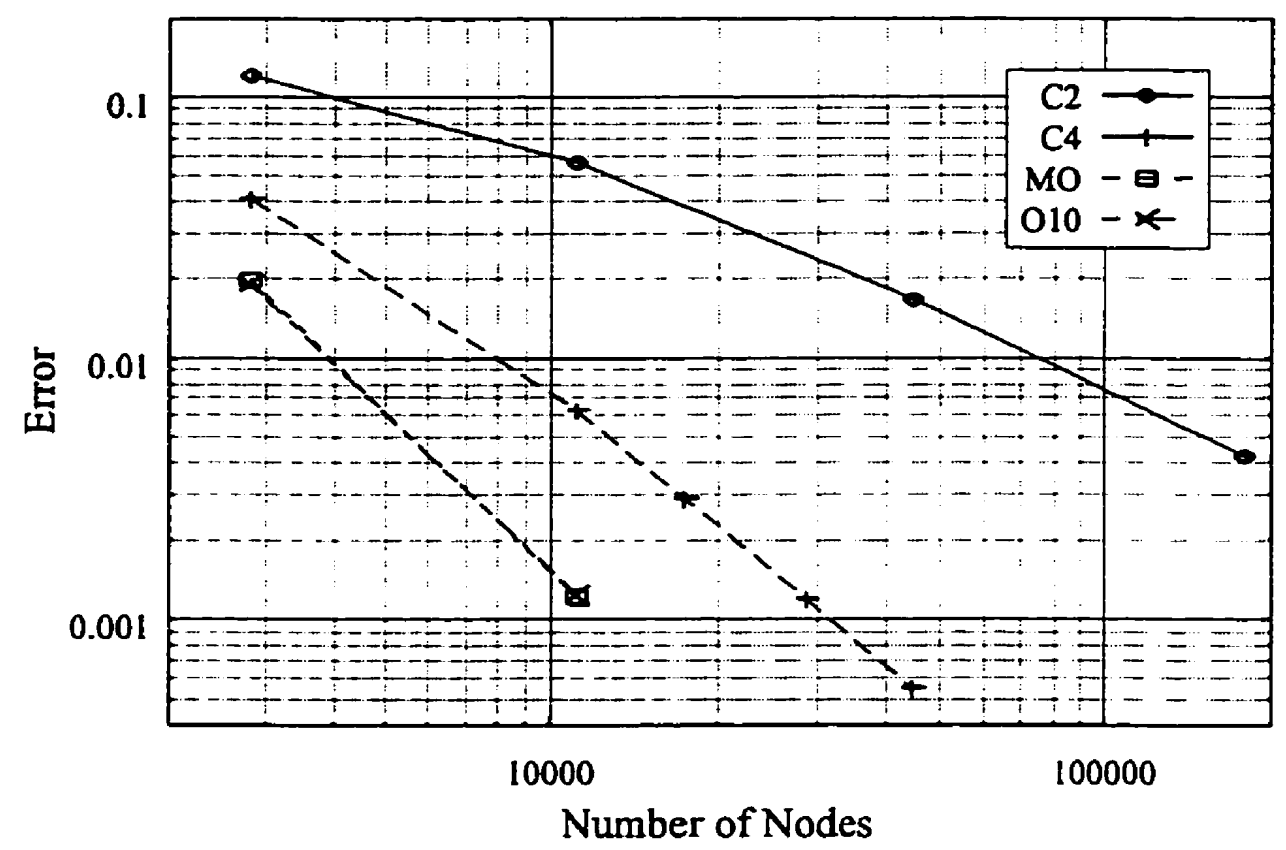

Figure 24: Normalized $L_{2}$ norm of the error in the electric field intensity, for the dielectric square case, as a function of the number of nodes in the grid

$$
\text { Error }=\sqrt{\frac{\sum_{i=1}^{n}\left[\left(E_{z}-E_{z}^{R e r}\right)_{i}\right]^{2}}{n}}
$$

where the sum is over the $n$ nodes in the numerical domain, and $E_{z}^{\text {Ref }}$ represents the value of $E_{z}$ obtained using the reference solution. Figure 25 plots these errors as a function of the computer time needed to obtain the solution. The time is measured in terms of the number of CPU cycles needed by a reference computer system to complete the simulation. It is clear that the high-accuracy schemes (MO and O10) are more efficient than the C4 and C2 schemes. To obtain the accuracy of the results generated by the MO and $\mathrm{OlO}$ methods on a grid with 11,200 nodes, the $\mathrm{C} 4$ scheme needs to be run on a grid with 2.5 times as many nodes, and requires twice as much CPU time. The results obtained using $\mathrm{C} 2$ on a grid with 16 times as many nodes gives an error which is about 3.5 times as large as that obtained by the MO scheme, taking about 25 times longer to run.

The above simulations illustrate the usefulness of the high-accuracy schemes. The method for handling the interface between two dielectric materials is also shown to work successfully. This can be seen in Figure 22 where the contours pass smoothly through the 
interface. The high-accuracy schemes are also clearly much more efficient than the lower order schemes. Even though the high-accuracy schemes take longer to run per grid node per time-step than the lower order schemes, the same error level can be obtained by using a grid with much fewer nodes, and, as a result, a larger time-step. This results in the simulation being completed much faster. Being able to use a grid with fewer nodes also results in a large savings in memory. The savings in both time and memory will be even more evident in three dimensions.

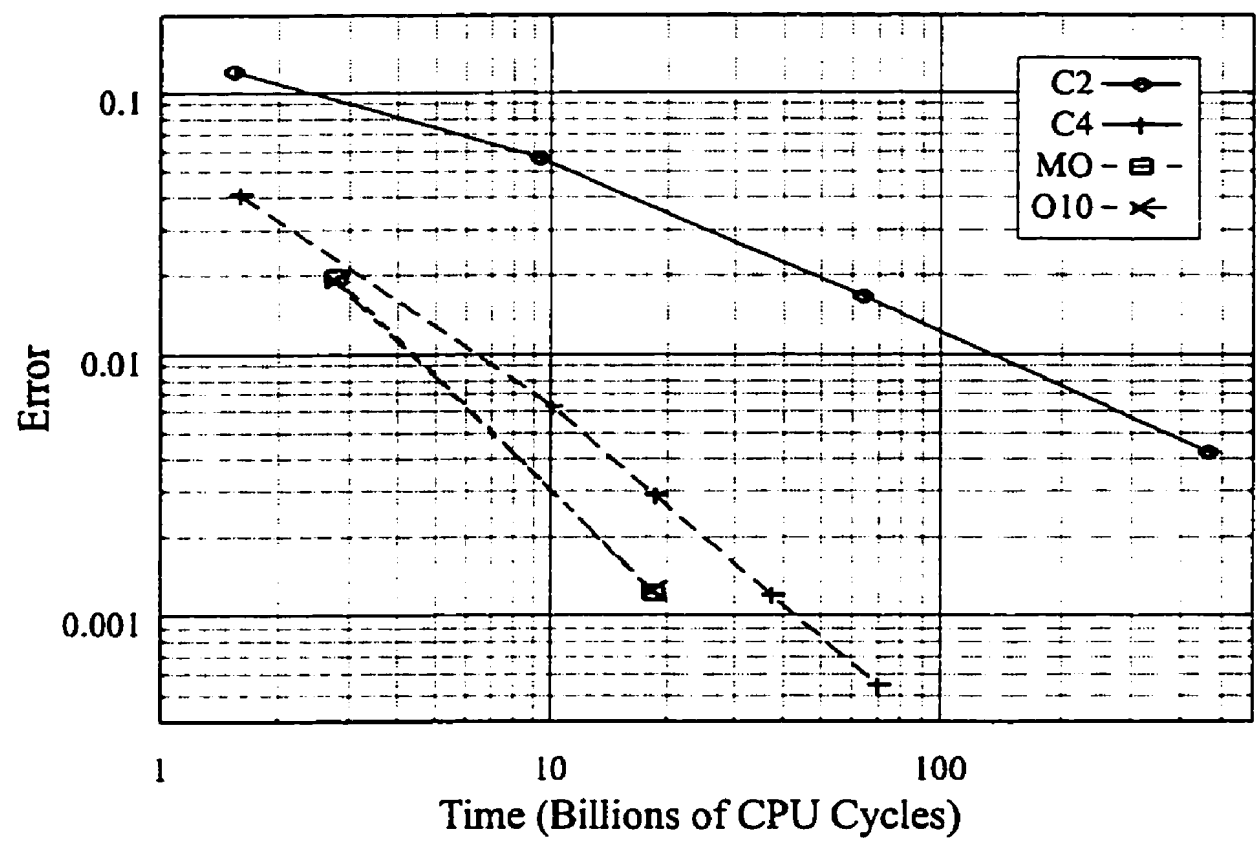

Figure 25: Normalized $L_{2}$ norm of the error in the electric field intensity, for the dielectric square case, as a function of the CPU cycles necessary to complete the simulation

In the above simulations, the optimized method (O10) gives the same results as the MO scheme. There are a number of reasons which explain this. First, the incident pulse has significant low wavenumber content. The majority of the Fourier components of the pulse used in the simulations have wavenumbers which give a value of $\kappa \Delta x<0.35$ on the grid with 11,200 nodes. These components are resolved more accurately by the MO scheme, as can be seen by inspecting Figures 9 and 10. Another factor which comes into play is that the numerical boundary formulation which is used near the interface between free space and the dielectric is the same in both the MO and 010 methods. As was shown in [91], the NBSs, in conjunction 
with the jump conditions, can have a very significant impact on the overall error. The errors introduced when the pulse is near the interface can become dominant, resulting in the accuracy gained by replacing the MO scheme with the 010 scheme being wasted. This last effect is reduced if the distance of travel between interfaces and boundaries is relatively large.

\subsection{Perfectly-Conducting Cylinder}

Several tests are now considered which use a curvilinear grid. These tests simulate a number of different waves incident on a perfectly-conducting cylinder. Figure 26 shows the geometry of the problem. The cylinder has a radius of unity, and the outer limit of the numerical domain is at $r=5$. Again, a nondimensional set of the equations are used which give a wave speed of one. The waves enter the domain from the left side and are incident at an angle of zero degrees. Grids which are made up of three distinct blocks are used. These grids, an example of which is shown in Figure 26, limit the widening of individual cells when moving away from the scatterer by doubling the resolution of the grid, in the $\theta$ direction, whenever the value of $r$ is doubled. This insures that the waves are evenly resolved over the entire domain.

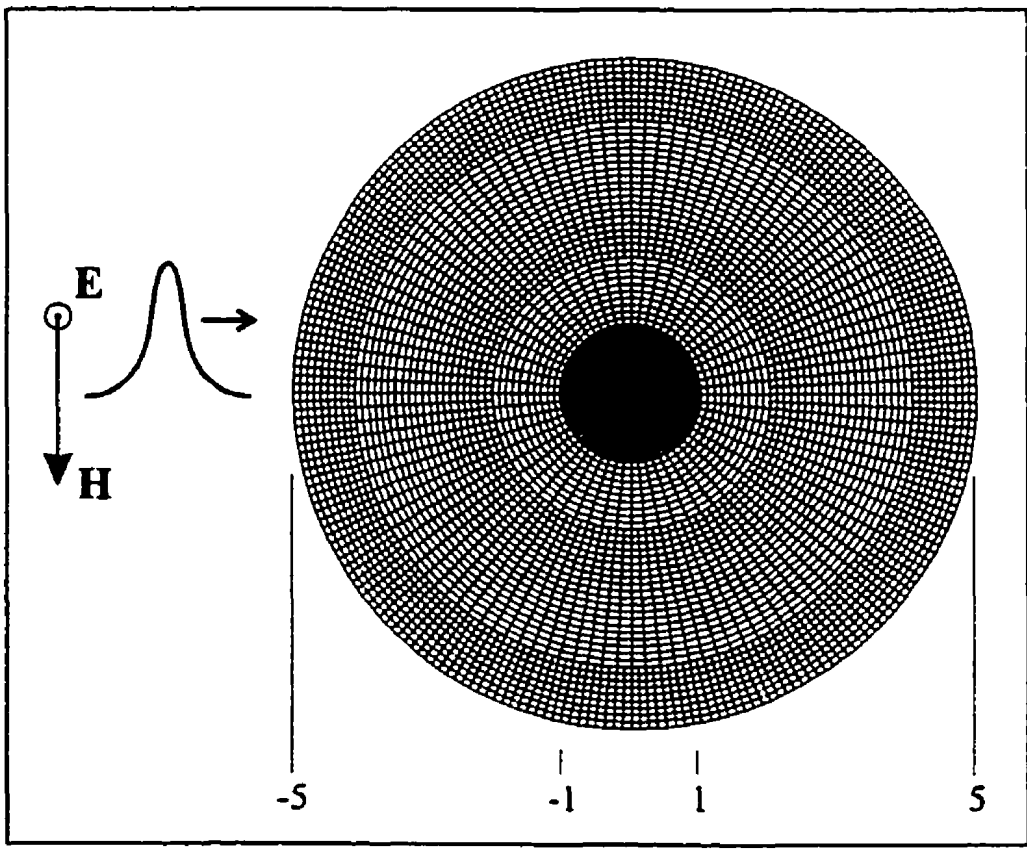

Figure 26: Example grid showing a pulsed plane wave with a Gaussian cross-section incident on a perfectlyconducting cylinder 


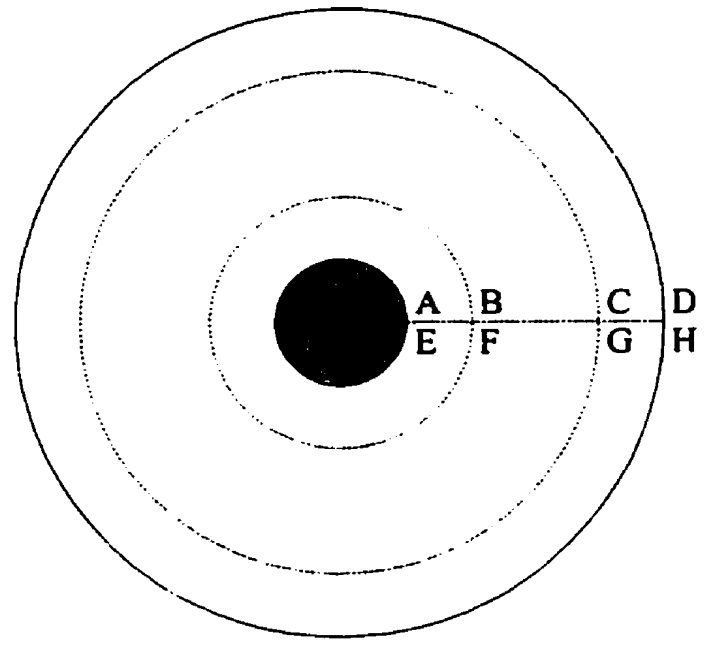

Physical Domain

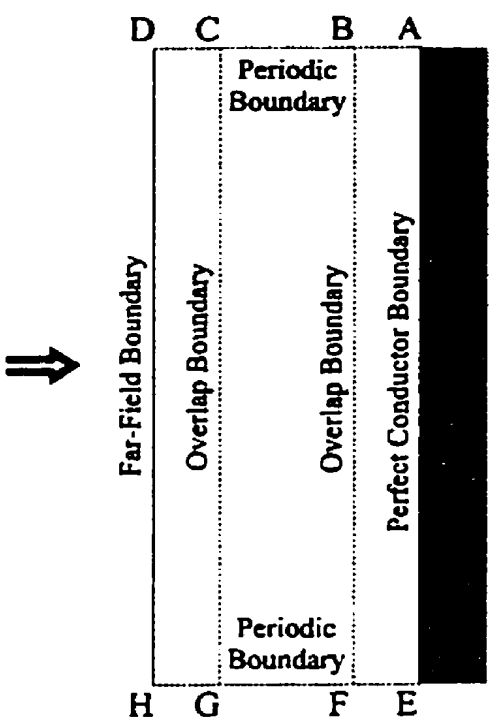

Computational Domain

Figure 27: Transformation of physical domain into computational domain showing different types of boundaries for a perfectly-conducting cylinder

Figure 27 shows a representation of the generalized coordinate transformation, described in $\S 2.3$, which maps the physical domain into the computational domain. The different types of boundary conditions are also shown in the computational domain. Each block is surrounded by a region of ghost points. The existence of these ghost points allow the grid metrics to be calculated numerically, using the same antisymmetric operator which is used in the calculation of the spatial derivatives of the electromagnetic fields, without resorting to the use of NBSs. The use of boundary operators can also be avoided when calculating spatial derivatives of field values near the interface between blocks. A result of using the ghost points is that the blocks overlap slightly, as shown in Figure 28. Data is passed between the blocks, moving from the regular nodes of one to the ghost points of the other. The fifth-order polynomial interpolation scheme, given by equation ( D.1) in Appendix D, is used to obtain the field values at nodes which do not have partners in the block being overlapped, as shown in Figure 28. The method described in $\S 4.2$ is used to handle the numerical computations at the surface of the perfect conductor. The grids are generated to obtain cells which have an aspect ratio close to one near this surface. When the aspect ratio deviates too much from this value, it is difficult 
to find boundary schemes which are stable and accurate for the high-accuracy methods. For all the tests, the time step is chosen so that the Courant number falls in the range 0.5 to 1.0 .

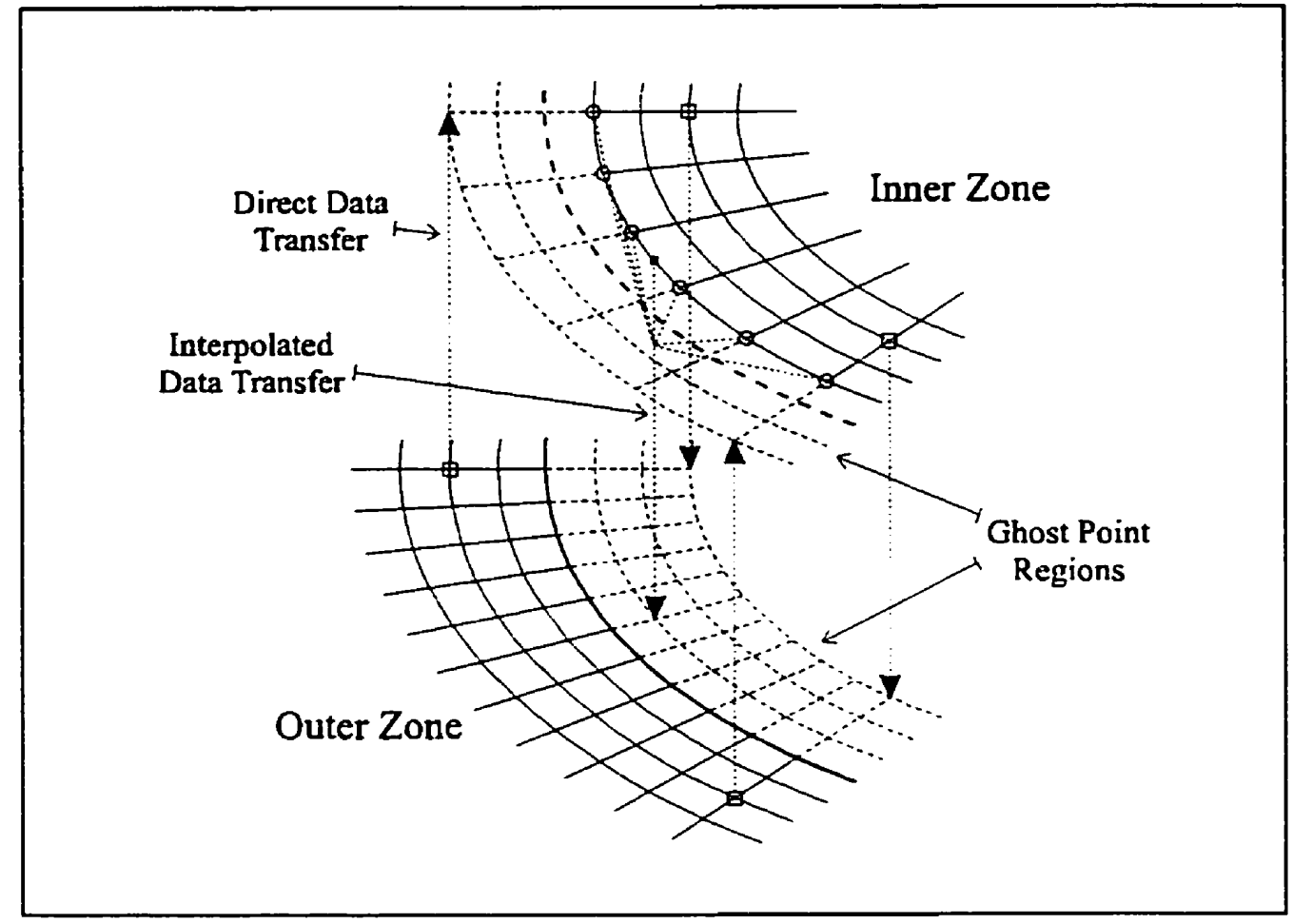

Figure 28: Overlapping grids showing example points at which data can be passed directly, and points at which interpolation must be used

\subsubsection{Gaussian Incident Pulse}

This test models a pulsed plane wave with a Gaussian cross-section incident on the cylinder described above. The incident electric field intensity is given by

$$
E_{z}(x, y, t)=\exp \left[-\frac{1}{2 \sigma^{2}}\left(x+\frac{15}{2}-t\right)^{2}\right]
$$

with $\sigma=0.3$, and the simulation is run until $t=8.5$. The FFBC based on the characteristic splitting is used for these tests. This method is adequate because the scattered field does not interact with the outer boundary during the simulation. Figure 29 shows contour plots of the electric field intensity for four different cases. Figure 29 a) shows a reference solution which is calculated using the MO scheme on a grid with 345,600 nodes. The results computed using the MO scheme on a grid with 5,400 nodes are shown in Figure 29 b). Even though this grid 


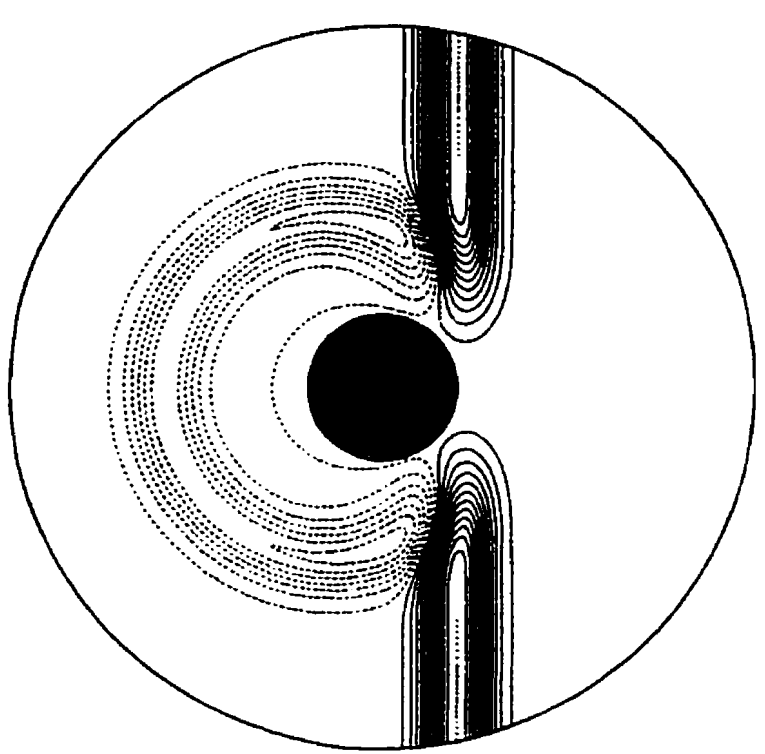

a)

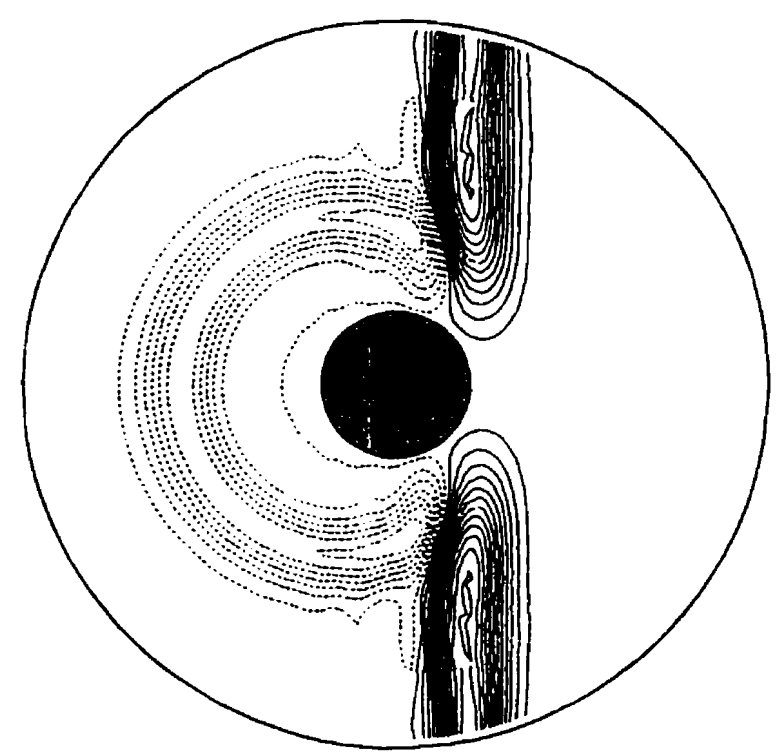

c)

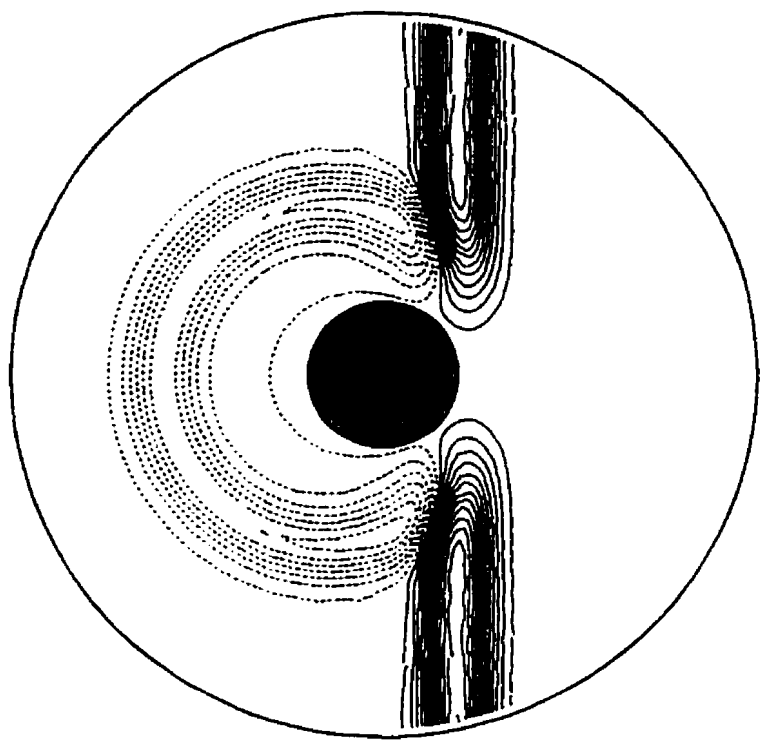

b)

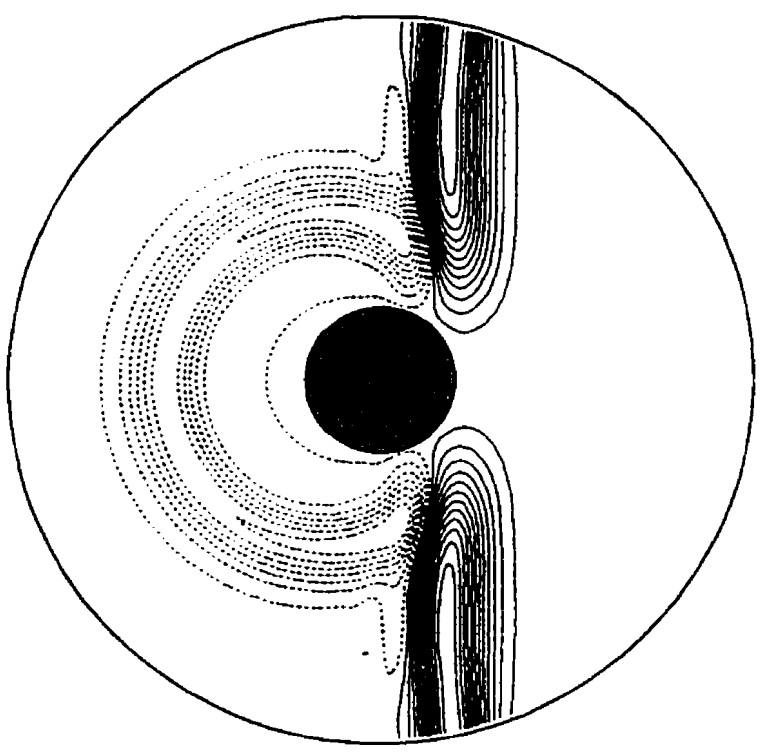

d)

Figure 29: Contour plots of electric field intensity for a) reference solution, b) MO using a grid with 5,400 nodes, c) C4 with 5,400 nodes, and d) C2 with 21,600 nodes

is fairly coarse, the solution obtained compares favorably with the reference solution. The jaggedness of the contour lines, and the discontinuities near the interface between the outer and center blocks, are artifacts of the plotting package. The numerical solutions are smooth. Plots of the solutions obtained using $\mathrm{C} 4$ on the grid with 5,400 nodes and C2 on a grid with 21,600 nodes are shown in Figures $29 \mathrm{c}$ ) and d), respectively. Both of these results deviate 
significantly from the reference solution. A description of all the grids used in the tests can be found in Table 4.

\begin{tabular}{|c|c|c|c|}
\hline Total Nodes & $\begin{array}{c}\text { Resolution in } \\
\text { Inner Block }\end{array}$ & $\begin{array}{c}\text { Resolution in } \\
\text { Middle Block }\end{array}$ & $\begin{array}{c}\text { Resolution in } \\
\text { Outer Block }\end{array}$ \\
\hline 5,400 & $10 \times 60$ & $20 \times 120$ & $10 \times 240$ \\
\hline 21,600 & $20 \times 120$ & $40 \times 240$ & $20 \times 480$ \\
\hline 31,104 & $24 \times 144$ & $48 \times 288$ & $24 \times 576$ \\
\hline 62,424 & $34 \times 204$ & $68 \times 408$ & $34 \times 816$ \\
\hline 69,984 & $36 \times 216$ & $72 \times 432$ & $36 \times 864$ \\
\hline 86,400 & $40 \times 240$ & $80 \times 480$ & $40 \times 960$ \\
\hline 345,600 & $80 \times 480$ & $180 \times 960$ & $80 \times 1,920$ \\
\hline
\end{tabular}

Table 4: Grids used for perfectly-conducting cylinder tests, where resolutions are given as radial nodes $\mathrm{x}$ angular nodes

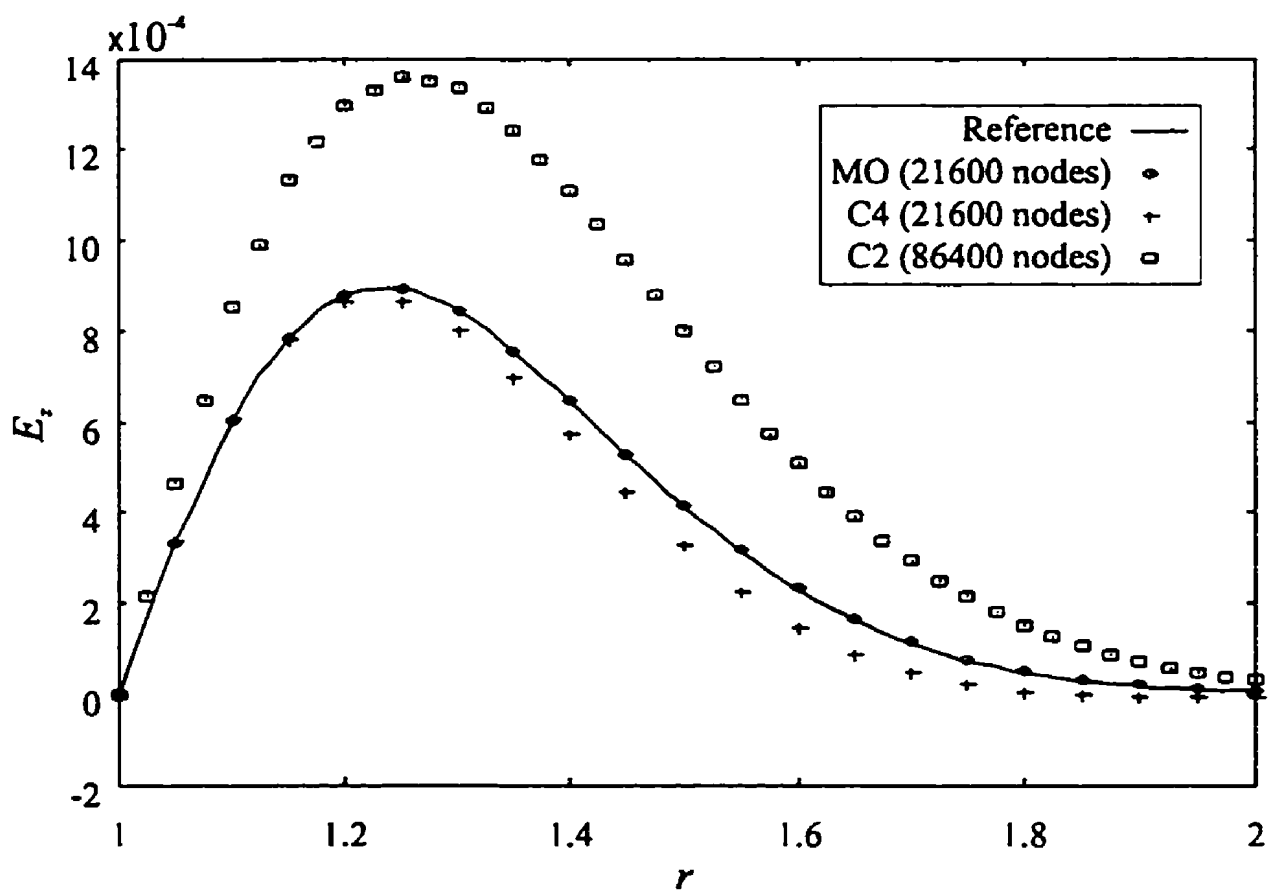

Figure 30: Electric field intensity along a radial line behind the perfectlyconducting cylinder for an incident pulse with a Gaussian cross-section

The electric field intensity along the positive $x$-axis, behind the perfect-conductor, is shown in Figure 30. The solution in this region is not visible in Figure 29 and is very sensitive to numerical errors. The reference solution, the results from the MO scheme on a grid having 21,600 nodes, the results from the C4 scheme on a grid with 21,600 nodes, and the results 
from the C2 scheme on a grid with 86,400 nodes are all shown. The solution obtained using the MO scheme is very accurate. The C4 method, when used with the same grid, produces results which deviate significantly from the reference solution. The solution obtained by using the C2 method on a grid with four times as many nodes is not adequate for engineering purposes.

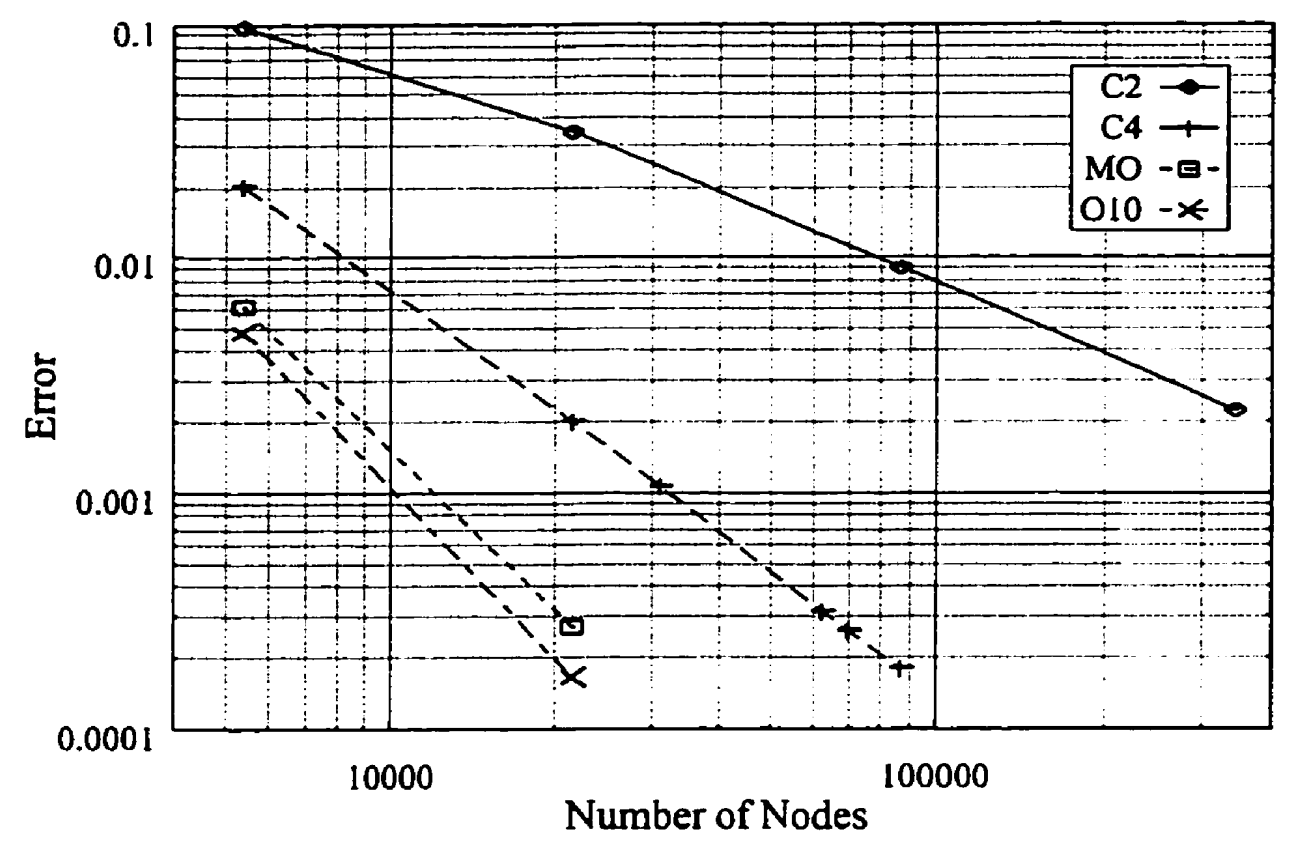

Figure 31: $\mathrm{L}_{2}$ norm of the error in the electric field intensity as a function of the number of nodes in the grid for a pulsed plane wave incident on the perfectly-conducting cylinder

Figure 31 shows the normalized $L_{2}$ norm of the error in the electric field intensity as a function of the grid resolution, and Figure 32 shows this error as a function of the CPU usage. The error value for each test is calculated using ( 5.2 ) with a reference solution obtained by running the MO scheme on a highly resolved grid. The trends shown in these figures are very similar to those obtained for the dielectric square case. To attain the same error level as that generated using the MO scheme with a grid having 21,600 nodes, the C4 scheme must be used on a grid with about 3.25 times as many nodes, and it takes about 3.4 times as long to complete the simulation. Even when using a grid with 345,600 nodes, the $\mathrm{C} 2$ method does not produce a solution which can compare with those computed by the high-accuracy methods on a grid with 21,600 nodes. The scheme optimized for $10 \mathrm{PPW}$ is seen to produce slightly better results than the maximum-order scheme when using the same grid. For example, on the grid 
with 21,600 nodes, the 010 scheme generates a solution which results in an error that is $61 \%$ of that obtained by using the MO scheme. This result is obtained with no additional CPU expense, and with no extra cost in terms of memory, since the two schemes have the exact same computational stencils.

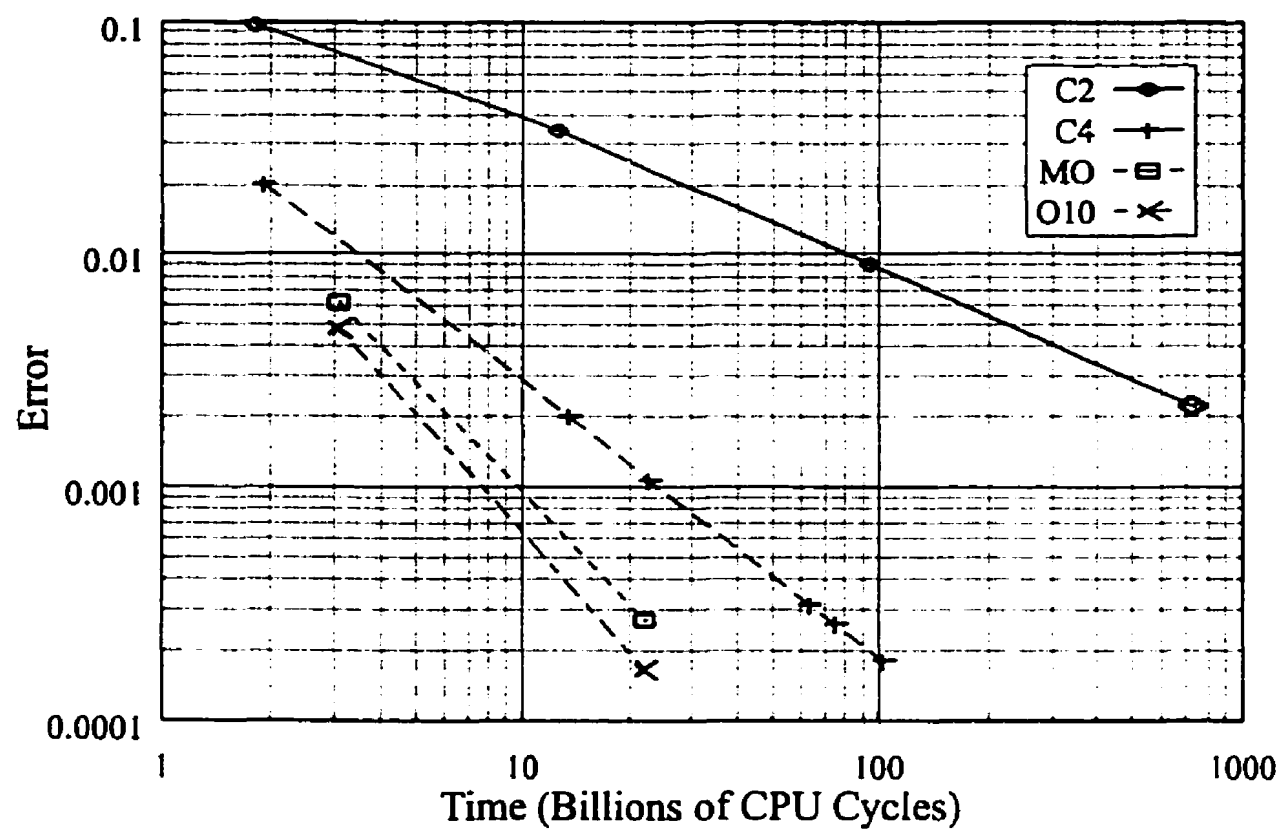

Figure 32: $\mathrm{L}_{2}$ norm of the error in the electric field intensity as a function of the number of CPU cycles it takes to complete the simulation for a pulsed plane wave incident on the perfectly-conducting cylinder

This simulation again illustrates the flexibility and efficiency of the high-accuracy schemes. They are successfully implemented on a complex curvilinear grid which is made up of several overlapping blocks. The method for handling the perfectly-conducting boundary condition also works extremely well. The optimized scheme gives better results than the MO scheme for this simulation, in contrast to the dielectric square case. One of the reasons for this is that the relative distance the pulse travels is slightly longer in the perfectly-conducting cylinder case. Also, the perfectly-conducting boundary condition does not introduce as much error as the dielectric-dielectric interface condition. This makes the error introduced while the wave propagates through the interior of the domain dominant, allowing the properties of the O10 scheme to be better displayed. It must be noted that the optimized scheme retains its ability to reduce the numerical errors, as compared to the MO scheme, when used on 
curvilinear grids. This result is seen even though the curvilinear coordinate transformation was not taken into account during the optimization of the scheme.

\subsubsection{Cosine Incident Wave}

Simulations of cosine plane waves incident on the perfectly-conducting cylinder are presented in this section. The existence of an analytical solution for a plane harmonic electromagnetic wave incident upon a perfectly-conducting circular cylinder is well known, and is discussed in detail in Appendix E. The first test is for an incident electric field intensity given by

$$
E_{z}(x, y, t)=\cos \kappa(x-t)
$$

with $\kappa=2 \pi$. A shaded contour plot of the analytical solution for the total electric field intensity resulting from this incident field is shown in Figure 33. When calculating the analytical solution, enough terms are used to converge the series to machine $\varepsilon$ in double precision. The test takes advantage of the fact that the solution for a harmonic wave incident on the cylinder

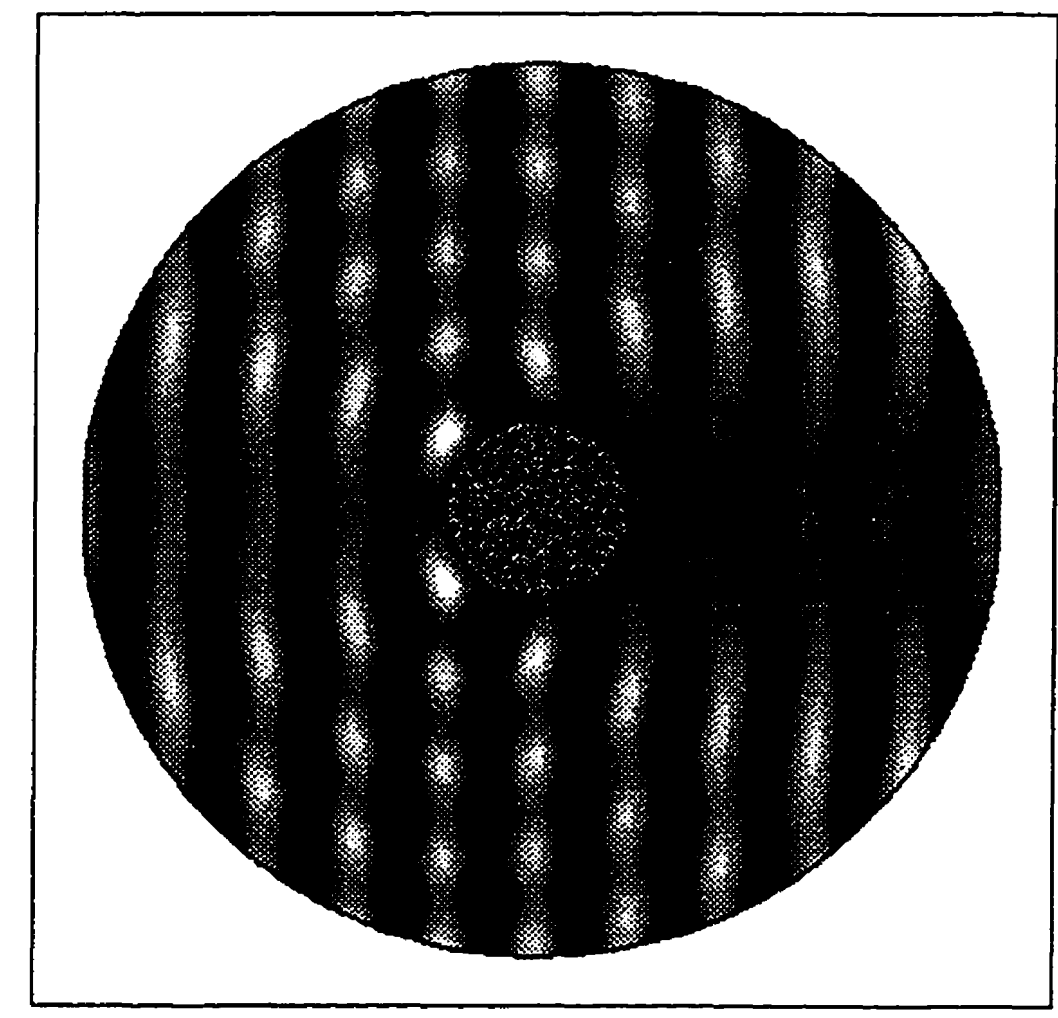

Figure 33: Electric field intensity for cosine wave, with $\kappa=2 \pi$, incident on a perfectly-conducting cylinder having radius of one 
is periodic in time. The field values in the domain are initially set to those given by the analytical solution at $t=0$, and the simulation is run until $t=8$. When the simulation is run for this length of time, all the initial disturbances that were present at $t=0$ have propagated out of the domain, and, for all the simulations run, a periodic steady-state solution has been reached. Because of the periodic nature of the fields, the solution should theoretically return to the initial conditions at this time. The continuous interaction with the scattered fields at the outer boundary stresses the far-field boundary conditions much more than the previous tests. Spurious reflections are a significant issue during the simulation, so the radiation FFBC, discussed in $\S 4.3 .1$, is used instead of the method based on characteristic splitting.

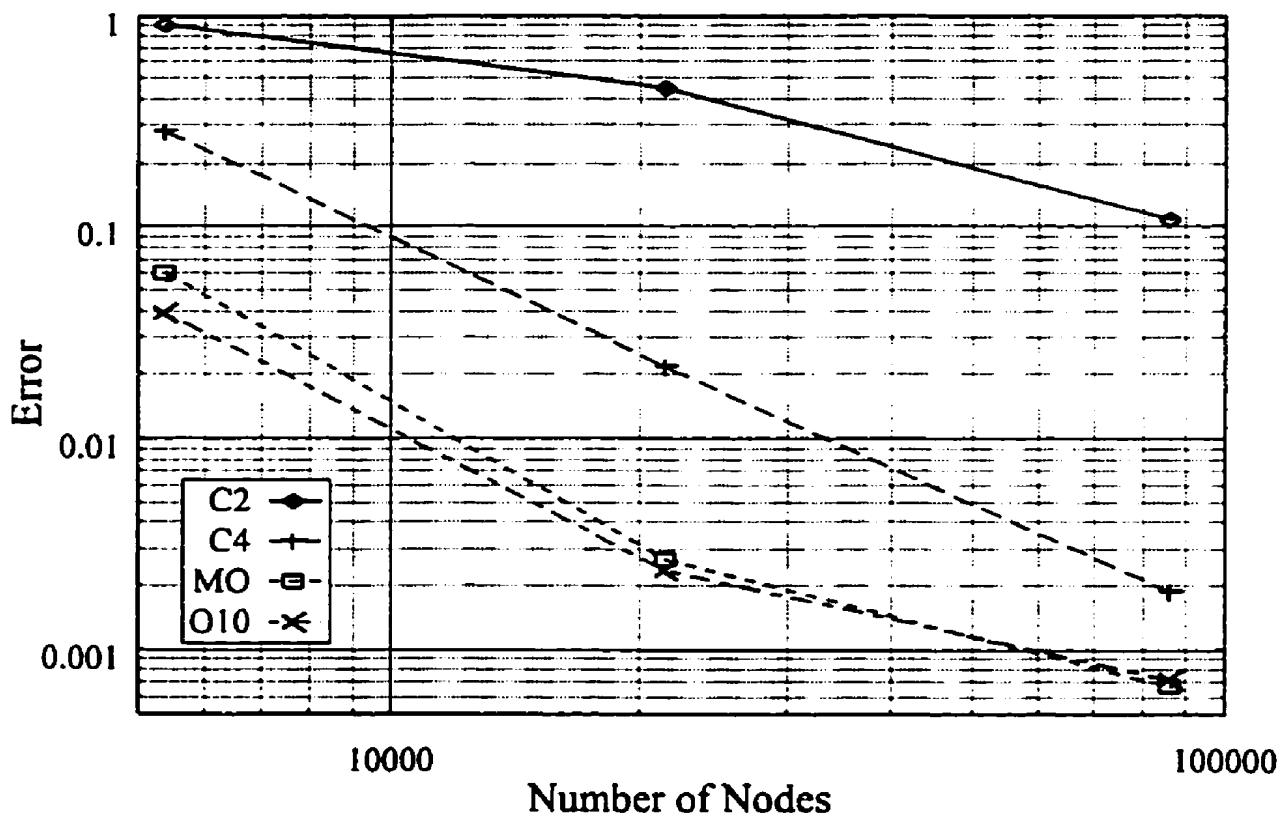

Figure 34: $L_{2}$ norm of the error in the electric field intensity as a function of the number of nodes in the grid for a cosine wave, with $\kappa=2 \pi$, incident on the perfectly-conducting cylinder

Figure 34 compares the errors, generated by the $\mathrm{C} 2, \mathrm{C} 4, \mathrm{MO}$, and $\mathrm{O} 10$ schemes, as a function of the number of nodes in the grids. Figure 35 plots these errors as a function of the CPU time needed to complete the simulations. Again, these errors are the normalized $L_{2}$ norm of the error in the electric field intensity as calculated by using ( 5.2 ). The analytical solution given in Appendix $\mathrm{E}$ is used as a reference for these calculations. The superior efficiency of the high-accuracy methods is clearly shown in these plots. The second-order method produces 


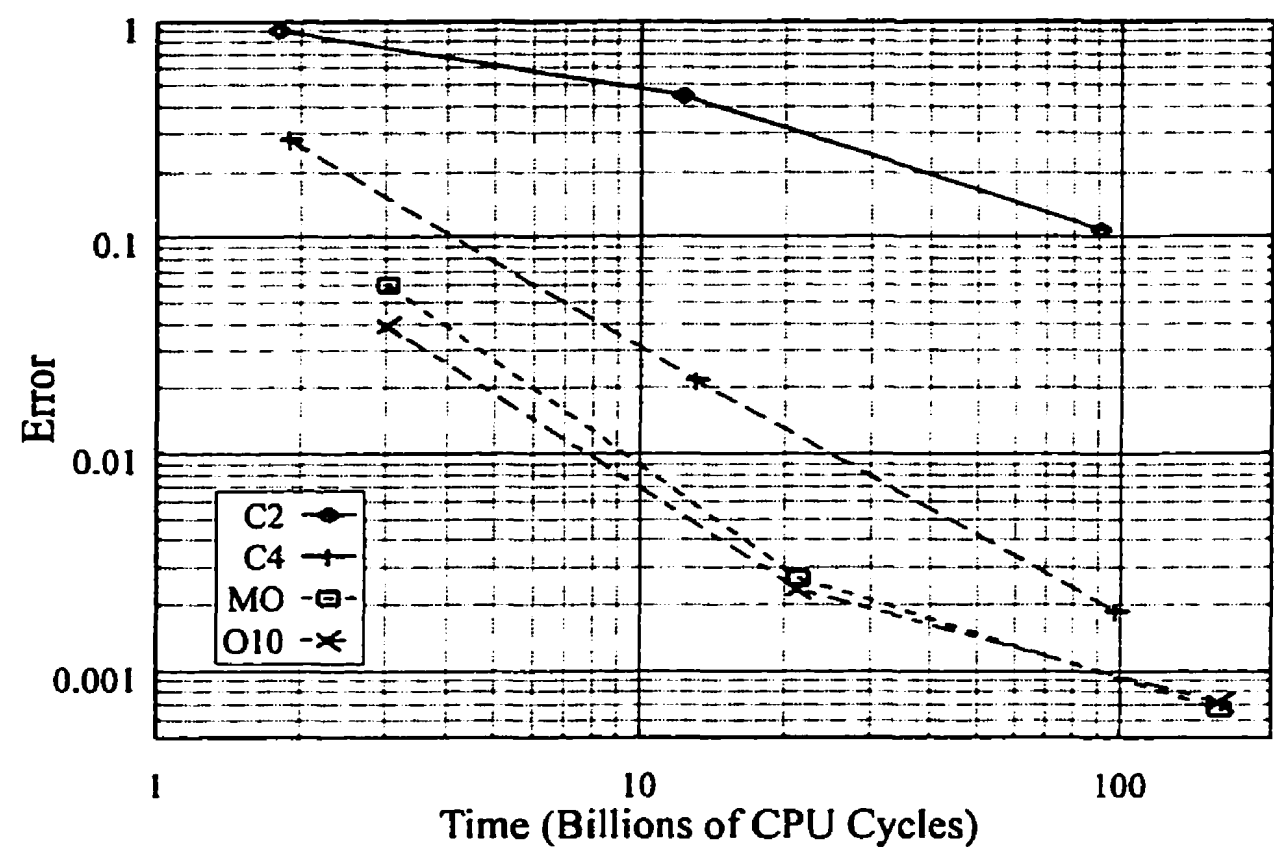

Figure 35: $L_{2}$ norm of the error in the electric field intensity as a function of the CPU time necessary to complete the simulation for a cosine wave, with $\kappa=2 \pi$, incident on the perfectly-conducting cylinder

solutions which give significant errors on all three of the grids. The fourth-order method needs to use the most resolved grid to generate a solution that gives acceptable accuracy, which can be produced by the high-accuracy methods on a grid with four times fewer nodes. Figure 36 shows the electric field intensity along a radial line directly behind the scatterer given by the analytical, fourth-order, and maximum-order solutions. The numerical solutions were obtained on a grid with 21,600 nodes. The solution obtained using the maximum-order method is considerably more accurate than that obtained using the fourth-order method.

The optimized scheme does not show significant improvement over the maximum-order scheme in the above tests because of the relatively short distance of travel for the waves being considered. To simulate cases where longer distances of travel are obtained (in terms of the number of wavelengths traveled) a highly resolved grid with 345,600 nodes is used, and incident waves with much higher wavenumbers are modeled. The first test has an incident wave with $\kappa=8 \pi$. The wave is resolved on this grid by at least 10 PPW and at most 20 PPW, and travels 32 wavelengths during the simulation. The analytical electric field intensity behind the 


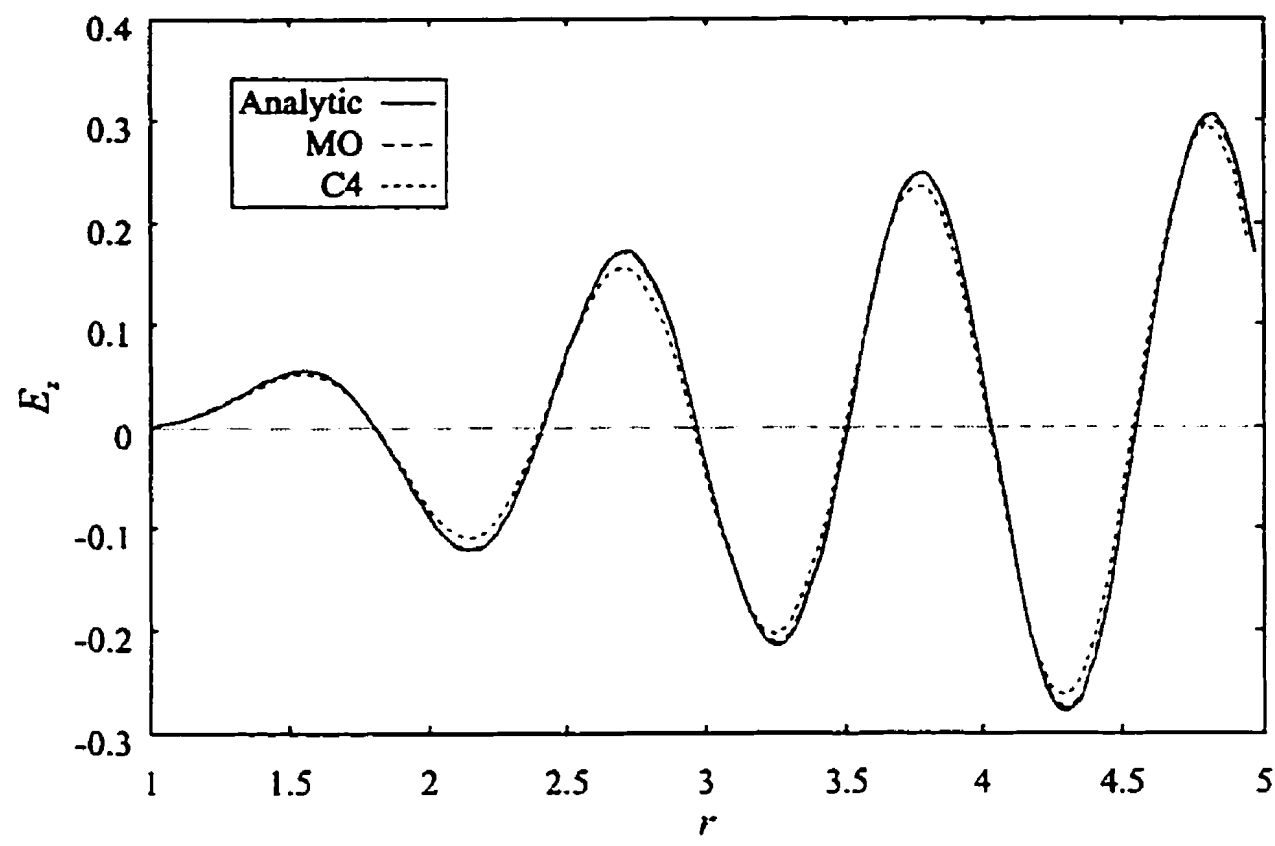

Figure 36: Electric field intensity along a radial line behind the perfectlyconducting cylinder for an incident cosine wave with $\kappa=2 \pi$

perfect conductor is shown in Figure 37 with the solutions generated by using the MO and 010 schemes. The solution given by the optimized scheme is essentially exact. The maximumorder scheme produces a solution which deviates slightly from the analytical solution. For this case, the optimized method produces a normalized $\mathrm{L}_{2}$ norm of the error in $E_{z}$ which is about $40 \%$ of that produced by the maximum-order scheme. The second test uses $\kappa=10 \pi$ for the incident wave. The wave is resolved by between 8 and $16 \mathrm{PPW}$, and the distance of travel is 40 wavelengths. For this wavenumber, the optimized method produces an error which is about $25 \%$ of that produced by the maximum-order scheme. Figure 38 shows a plot of the electric field intensity behind the perfect conductor, comparing the $\mathrm{MO}$ and $\mathrm{O} 10$ results to the analytical solution. For this case the maximum-order scheme generates a solution which deviates significantly from the analytical result. The optimized scheme gives a fairly good solution which only has very slight deviations from the analytical result. The above tests show the promise of the optimized method. The optimized method produces much better results than the maximum-order scheme when the number of wavelengths of travel is large. For the second test, the optimized method gives an error which is $1 / 4$ of that generated by the maximum-order scheme, 
while using the exact same computer resources. An even greater improvement would be seen for simulations in which the waves would have to travel even larger distances.

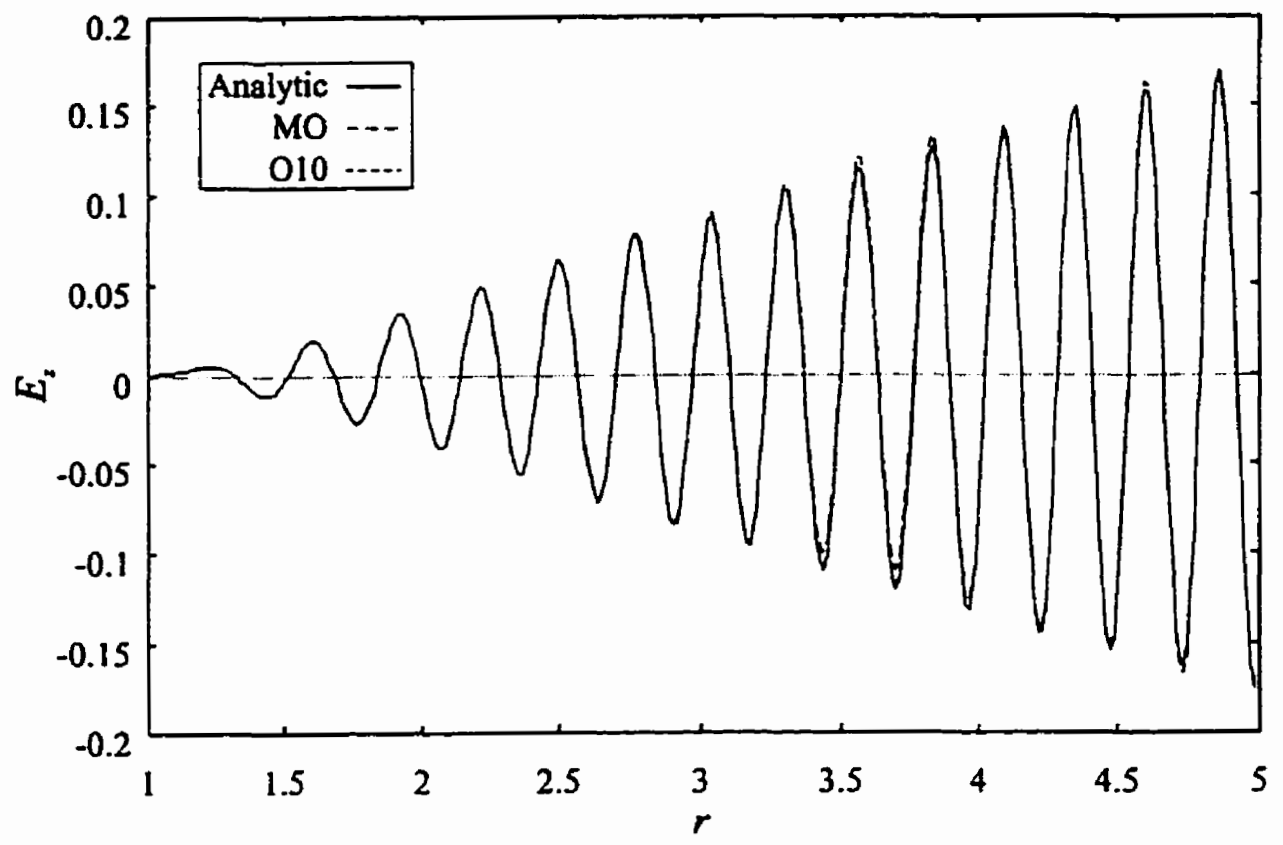

Figure 37: Electric field intensity along a radial line behind the perfectlyconducting cylinder for an incident cosine wave with $\kappa=8 \pi$

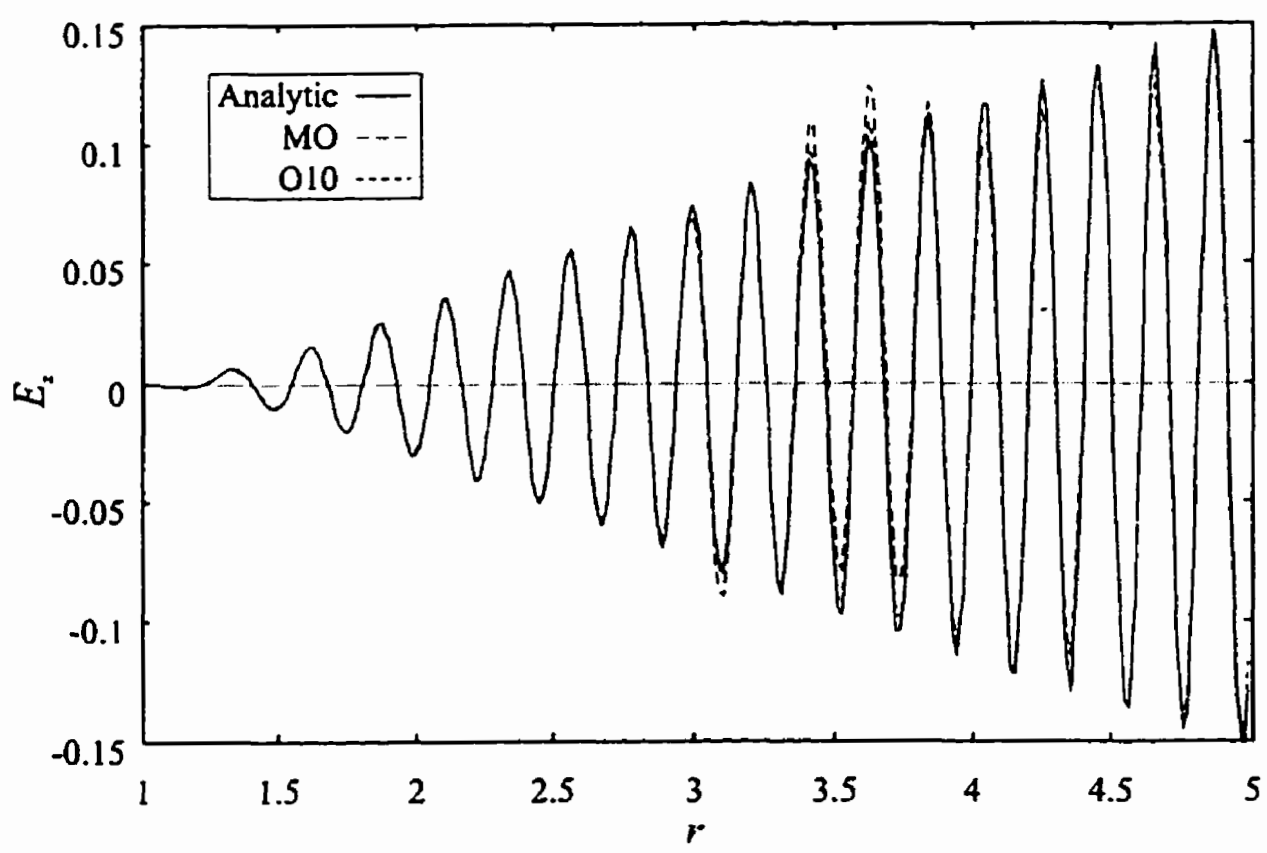

Figure 38: Electric field intensity along a radial line behind the perfectlyconducting cylinder for an incident cosine wave with $\kappa=10 \pi$ 


\subsection{Evaluation of Far-Field Boundary Conditions}

The far-field boundary conditions discussed in $\S 4.3$, including both the original and modified versions of the radiation FFBC, are implemented in Cartesian coordinates for the geometry shown in Figure 39. The numerical domain uses a square grid with a resolution of $50 \times 50$ nodes. A plane wave pulse with a Gaussian cross section enters the domain from the bottom left hand corner at an angle of $45^{\circ}$. The equation for the pulse is given by ( 5.1 ) where $\sigma=0.06$. The incident field is enforced along the domain's bottom and left boundaries, allowing the use of the interior operators right up to these boundaries. The different FFBCs are implemented along the top and right boundaries. The simulation is run using the MO scheme, at a Courant number of one, until the pulse travels across the entire domain and exits through the top right corner. The material properties of the domain are normalized so that the pulse travels at a speed of one. Theoretically the pulse should travel out of the domain and all the fields should return to zero. In practice, some spurious reflections are introduced.

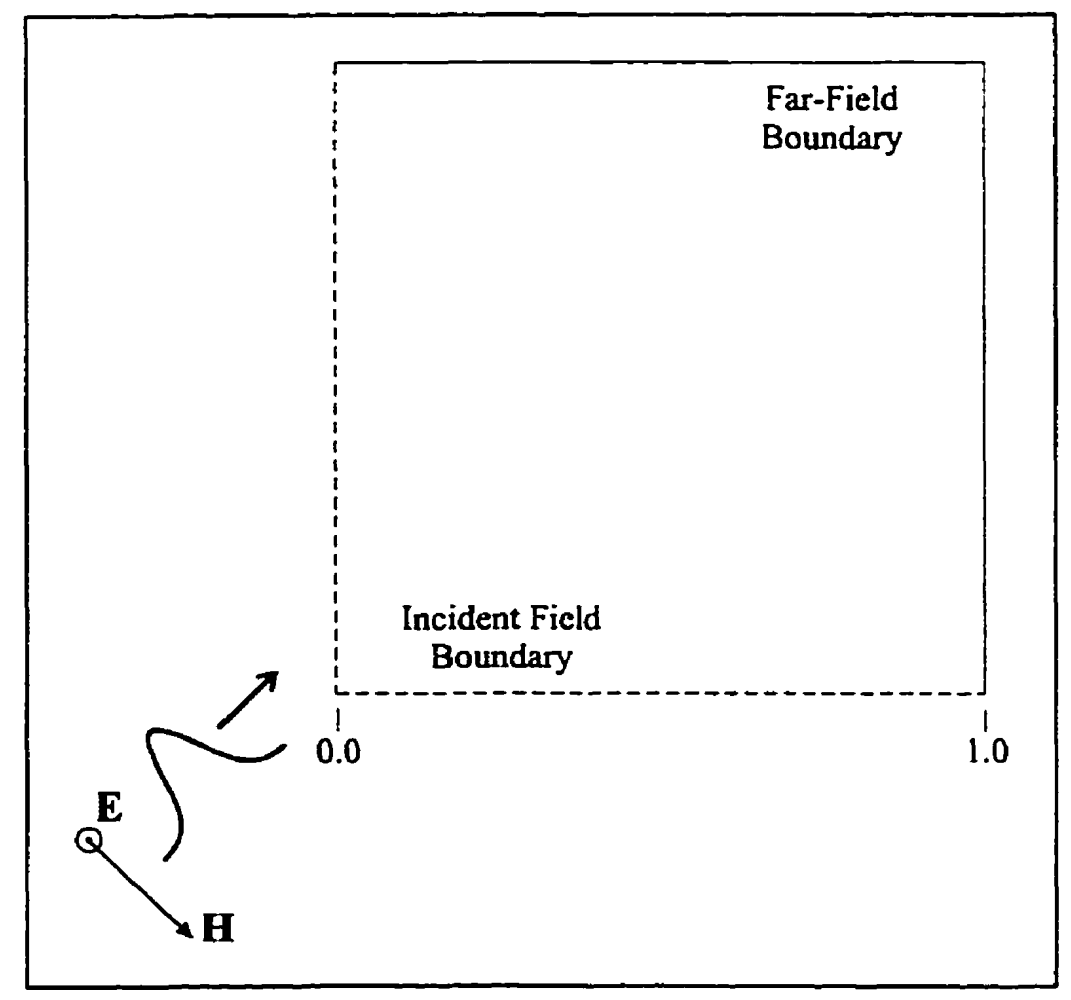

Figure 39: Geometry used to test different far-field boundary conditions in Cartesian coordinates 
Figure 40 shows a plot of the time-history of the $L_{2}$ norm of the $z$ component of the electric field intensity for the different FFBCs being considered. When the simulation reaches a time of $t=2$ the majority of the pulse should have exited the domain. Each of the FFBCs has a different level of success in damping out the unwanted spurious reflections. At this time, the characteristic method reduces the maximum value of the norm by a factor of about eight, the unmodified radiation method results in a factor of fifty reduction, and the modified radiation condition results in a factor of twenty-two reduction. It is interesting to note how each FFBC affects the fields after the incident pulse should have left the domain. The fields resulting from the spurious reflections all decay at different rates depending on the FFBC used. Surprisingly, the characteristic method results in the fastest decay. The modified radiation boundary condition causes the fields to decay faster than the original radiation method.

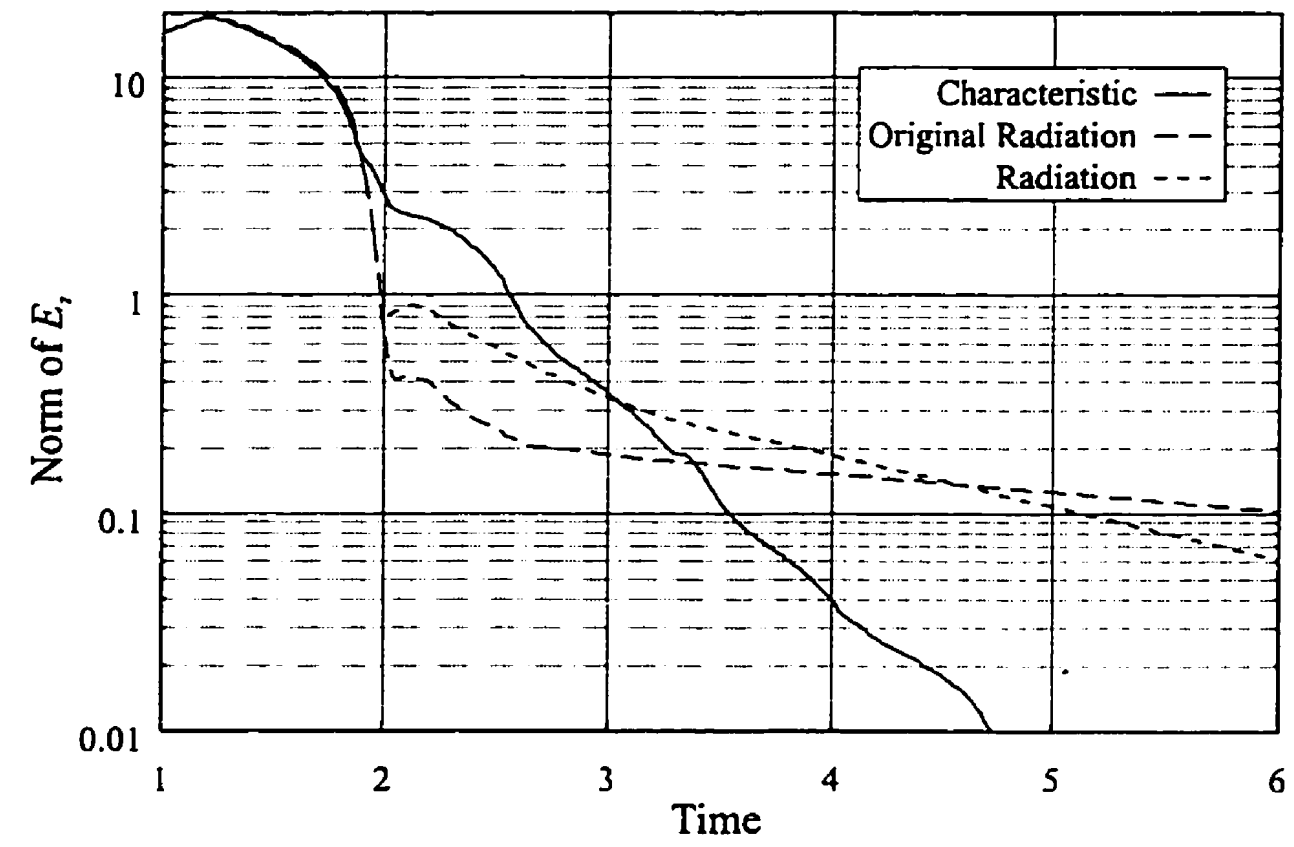

Figure 40: $L_{2}$ norm of the electric field intensity as a function of the time elapsed during the simulation, for a pulsed plane wave moving through a square domain, using different far-field boundary conditions

For most problems of interest the far-field boundary will be located close to some scattering object. For this type of problem one can expect that the spurious reflections will interact with the scatterer by $t=2.5$, at which time the reflections will have traveled half way back through the domain. The FFBCs that best model the physics of a wave exiting the domain, are 
those that generate the smallest norm by $t=2.5$. After this time, the rate at which the fields in the domain decay is a function of the numerics and is not an indication of how well the physics is being modeled. It is clear that the methods based on the radiation conditions generate spurious reflections which will have a much smaller impact on the solution. The method which produces the smallest spurious reflections is the original radiation far-field boundary condition. The modified radiation scheme still generates good results while accelerating the decay of the fields remaining in the domain, which is of primary importance when considering some grids in curvilinear coordinates.

To illustrate the effect of using a curvilinear grid, the three different FFBCs discussed above are used to model a pulsed plane wave incident on the perfectly-conducting cylinder. The problem is identical to that given in $\S 5.2 .1$, except the incident field is given by

$$
E_{z}(x, y, t)=\left(t-x-\frac{15}{2}\right) \exp \left[-\frac{1}{2 \sigma^{2}}\left(x+\frac{15}{2}-t\right)^{2}\right]
$$

where $\sigma=0.6$. After this pulse reflects off of the perfectly-conducting cylinder and exits the domain, the fields remaining near the surface of the cylinder are very small. This is an ideal case for testing the different FFBCs, because, as in the test given above, the norm of the electric field intensity should approach zero as the reflected pulse exits the domain. The MO scheme is used to model the problem on the grid containing 5,400 nodes.

A plot of the $\mathrm{L}_{2}$ norm of $E_{z}$ versus the time elapsed during the simulation is given in Figure 41 for the three different far-field boundary conditions. The trends shown are similar to that given in the Cartesian coordinate test, except that the slow decay of the fields generated by the original radiation boundary condition becomes an instability when this curvilinear grid is used. This figure clearly shows that the modified radiation condition results in a stable scheme which allows the reflected pulse to exit the domain with very little spurious reflection. This modified scheme allows much more of the reflected pulse to exit the outer boundary than the characteristic method, although the characteristic method causes the fields left in the domain to decay at a much faster rate. It should be noted that the trends shown in this test, and the Cartesian grid case, are identical when the C2 scheme is used. The instability introduced by the original radiation far-field boundary condition is present even when this lower-order method is used. 


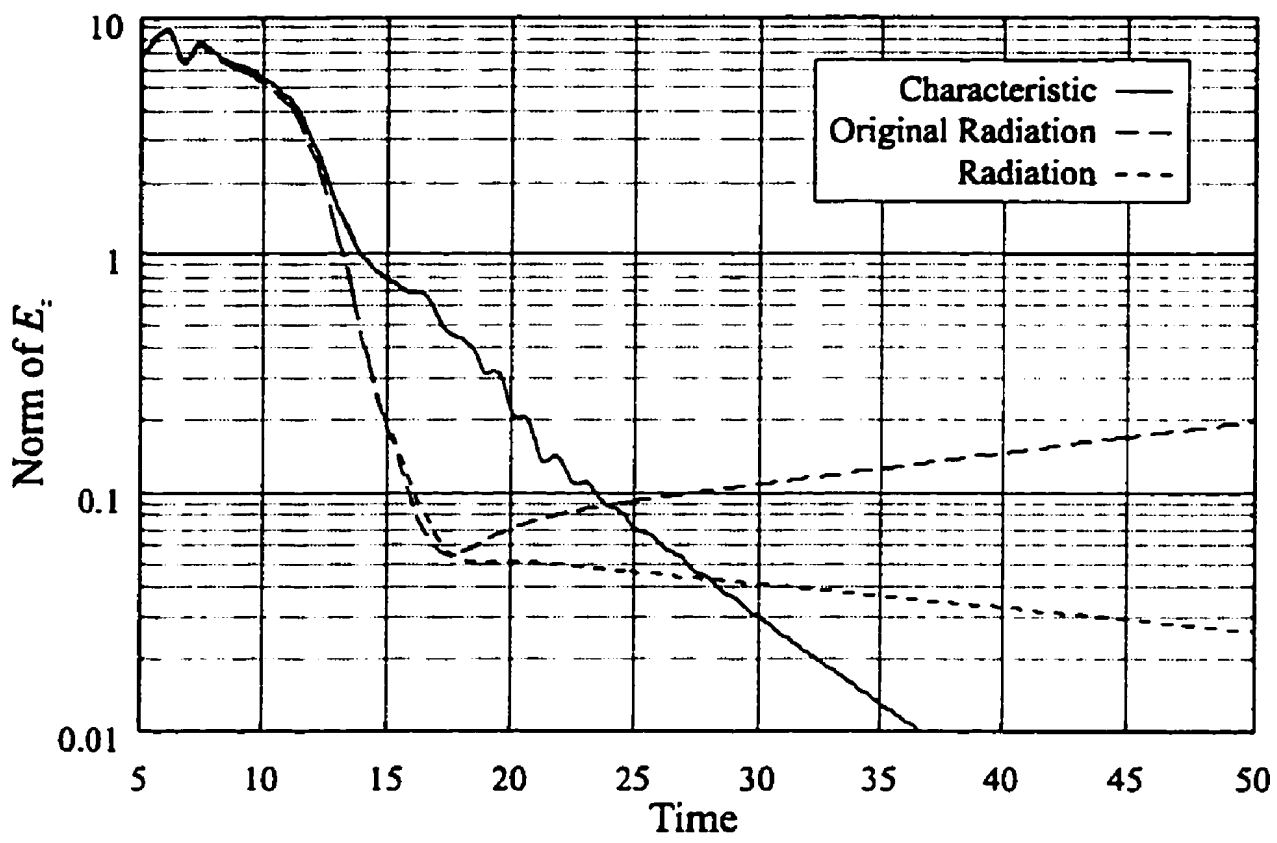

Figure 41: $\mathrm{L}_{2}$ norm of the electric field intensity as a function of the time elapsed during the simulation, for a pulsed plane wave incident on a perfectly conducting cylinder, using different far-field boundary conditions

To get an idea of the size of the errors introduced by using the different far-field boundary conditions, the problem of a cosine wave incident on the perfectly-conducting cylinder is studied, with $\kappa=2 \pi$, as described in $\S 5.2 .2$. The tests are run with a number of grid resolutions using the MO scheme in conjunction with three separate far-field boundary conditions. First, the interior operator is used right up to the outer boundary, and the analytical solution is used for nodes which lie outside the domain. Second, the locally one-dimensional characteristic splitting method is used, and finally, the radiation far-field boundary condition is imposed. The radiation FFBC refers to the modified scheme discussed in the tests given above.

Figure 42 shows the resulting normalized $L_{2}$ norm of the errors in the electric field intensity, calculated using the analytical solution as a reference, as a function of the number of nodes used in the grid. The characteristic method introduces significant errors due to spurious reflections. As the grid is resolved, the error does not get smaller than approximately 0.003 . The radiation condition performs very well, giving errors which are essentially identical to those produced by imposing the analytical solution outside the domain. The electric field intensity along the $x$-axis behind the cylinder is plotted in Figure 43 . This plot compares the 


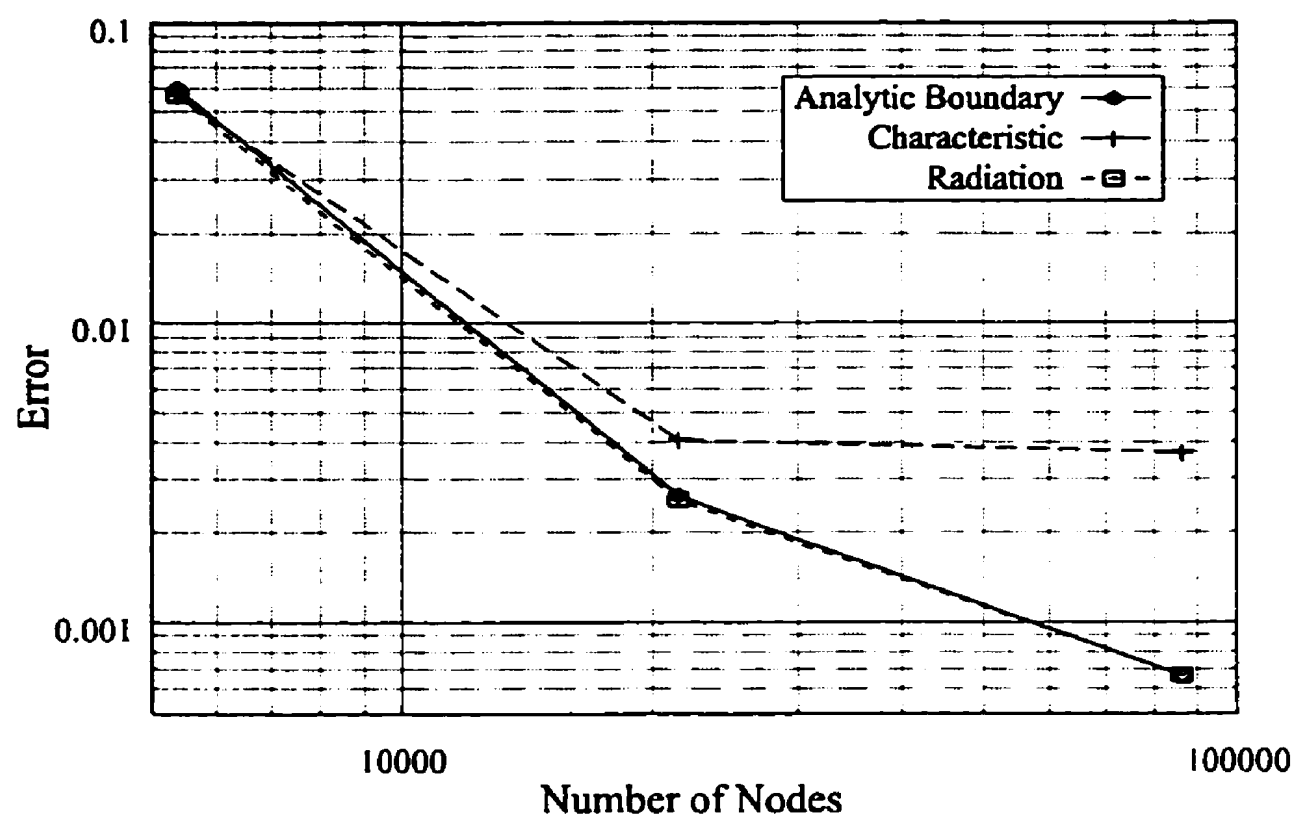

Figure 42: $L_{2}$ norm of the error in the electric field intensity as a function of the number of nodes in the grid, for a cosine wave, with $\kappa=2 \pi$, incident on the perfectly-conducting cylinder, using different far-field boundary conditions

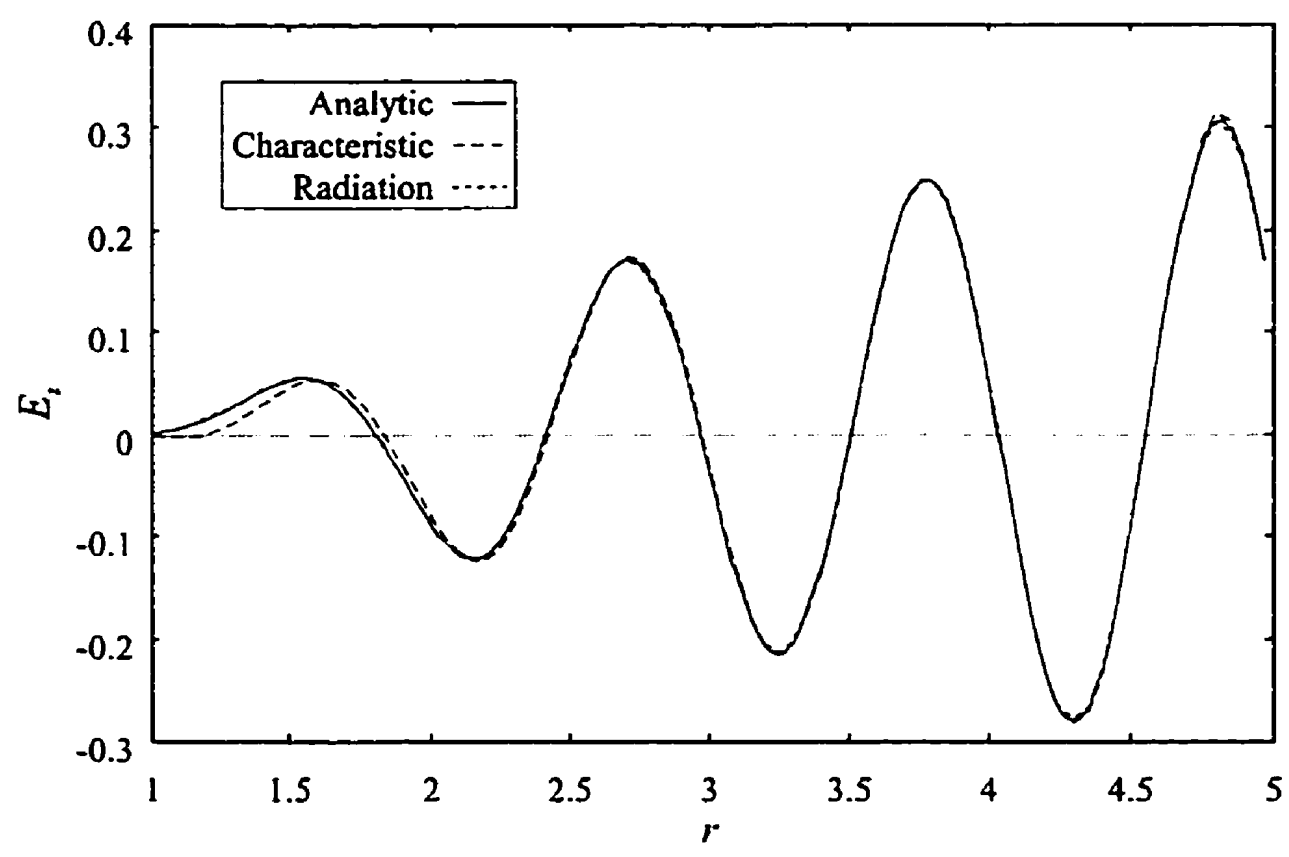

Figure 43: Electric field intensity along a radial line behind the perfectlyconducting cylinder, for an incident cosine wave with $\kappa=2 \pi$, using different far-field boundary conditions 
results for simulations using the characteristic splitting and radiation boundary conditions on a grid with 21,600 nodes to the analytical solution. The solution obtained using the radiation boundary condition is practically indistinguishable from the analytical solution. The effect of the spurious reflections due to the use of the characteristic splitting method is very evident especially near the perfect-conductor. Resolving the grid does not improve this result, because the solution is converging to a state which includes the spurious reflections. The performance of the radiation far-field boundary condition is more than adequate when used in conjunction with the high-accuracy methods, reducing the errors due to spurious reflections to a level well below the errors produced in the interior of the domain.

\subsection{Circular Dielectric Scatterer With a Perfectly-Conducting Core}

The final simulation models a pulsed plane wave with a Gaussian cross-section incident on a circular dielectric scatterer which has a perfectly-conducting core. This is an important case because all of the different types of boundary conditions considered are implemented on a complex curvilinear grid. The geometry of the problem is shown in Figure 44. The perfectlyconducting core has a radius of unity, the dielectric material extends from this core out to a radius of two, and the outer limit of the numerical domain is at $r=5$. The material properties of the free-space region are normalized to one, which gives a wave speed of unity in this region. The electrical permittivity of the dielectric is four, which results in a wave speed of $1 / 2$ in this region. The incident electric field intensity is given by ( 5.3 ) with $\sigma=0.3$. The grid used has 28,800 nodes and is shown in Figure 44 with every second node removed. The grid is made up of three separate blocks, two in the free-space region, which are used to limit the size of the cells near the outer boundary, and one for the dielectric region. The dielectric block uses a grid which has 40 nodes in the radial direction and 240 nodes in the angular direction, the middle block also has $40 \times 240$ nodes, and the outermost block has $20 \times 480$ nodes. The spatial resolution of the grid in the dielectric region is twice that of the grids used in the freespace region. This keeps the pulse well resolved over the entire grid.

Figure 45 gives a representation of the coordinate transformation which maps the physical domain into the computational domain. The different types of boundary conditions are also shown in the computational domain. The simulation is run using the MO scheme with a time 
step of 0.05 which results in the Courant number falling in the range 0.5 to 1.0 . The radiation FFBC is implemented along the outer boundary.

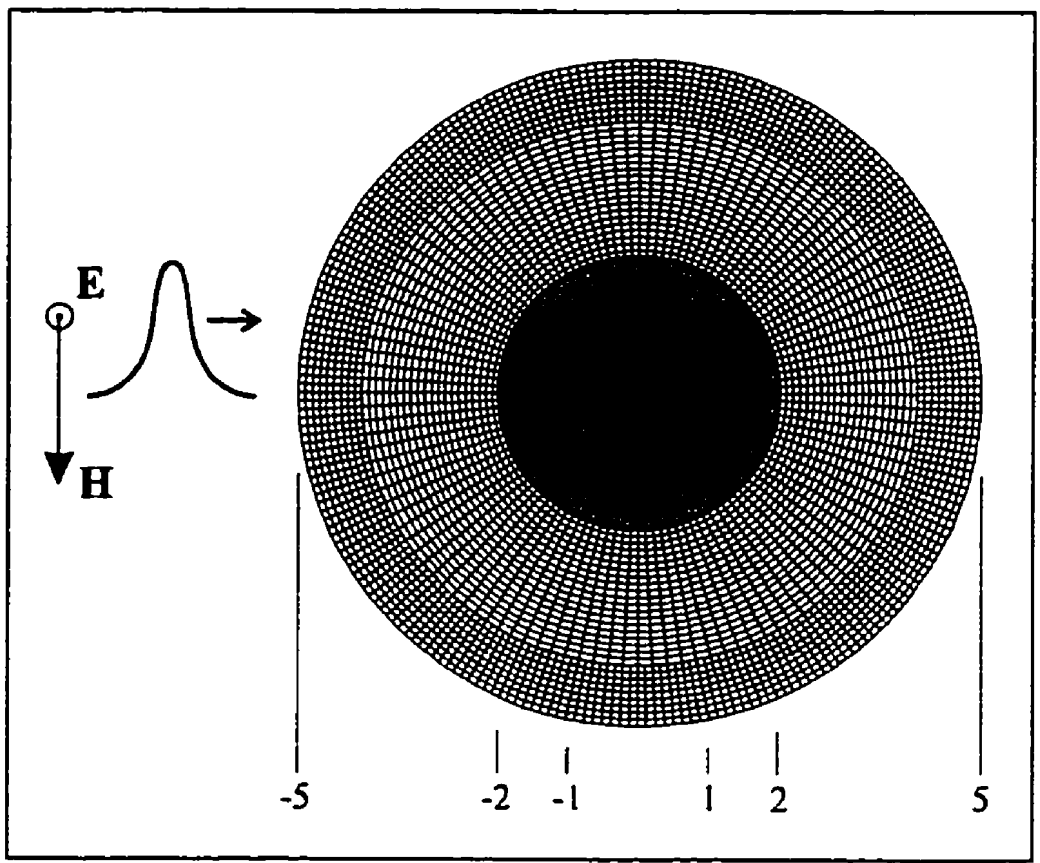

Figure 44: Example grid showing a pulsed plane wave incident on a circular dielectric scatterer with a perfectly-conducting core

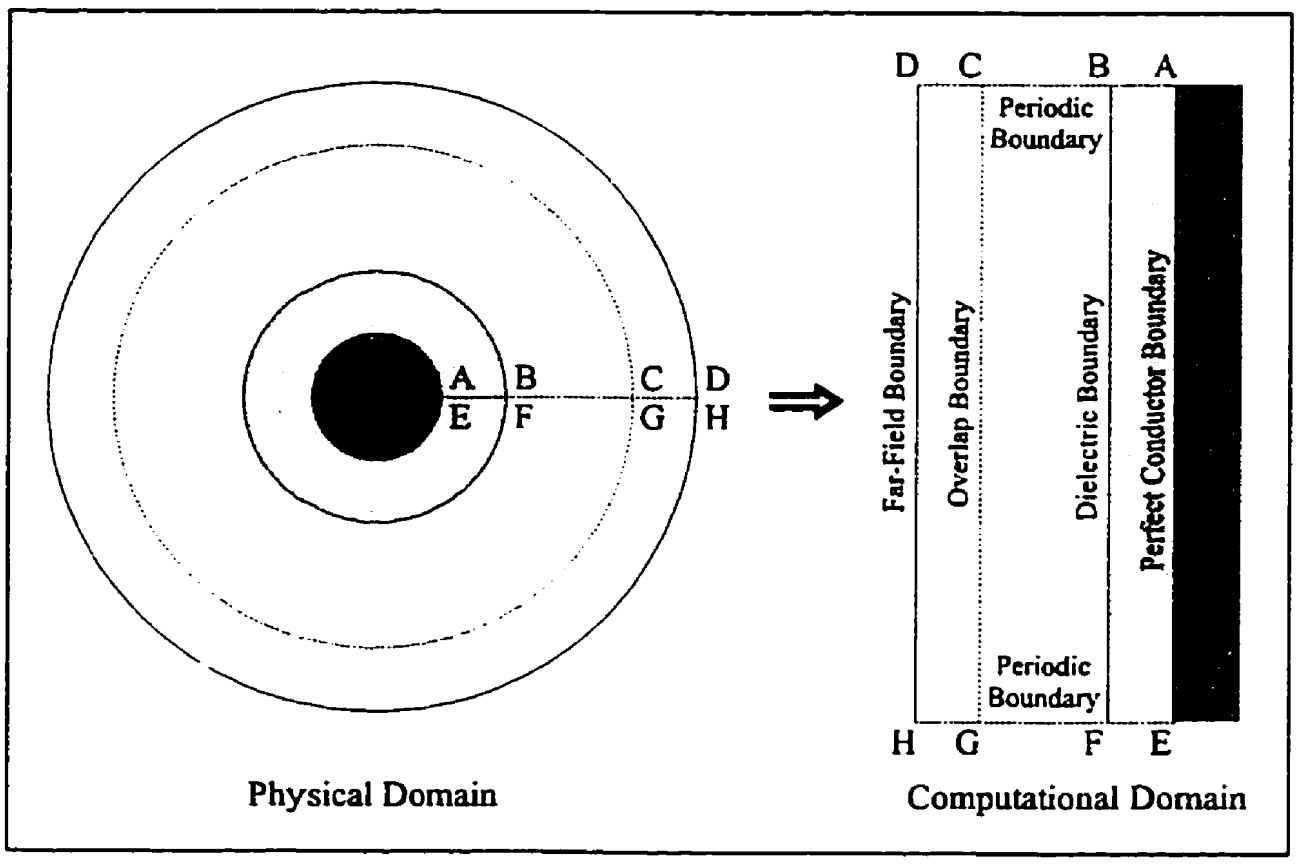

Figure 45: Transformation of physical domain into computational domain, showing different types of boundaries for a circular dielectric scatterer with a perfectly-conducting core 


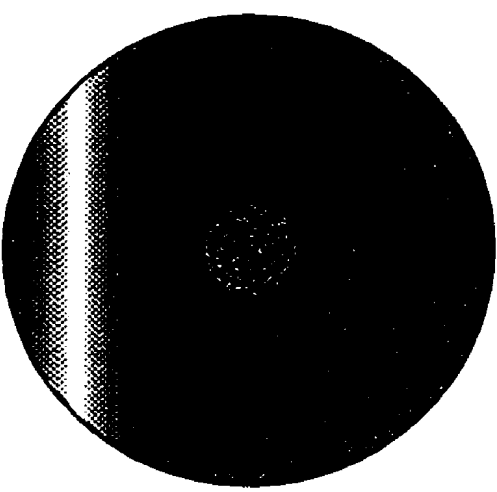

$t=4$

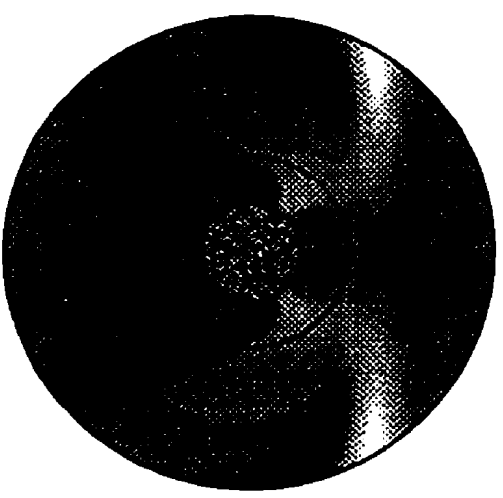

$t=10$

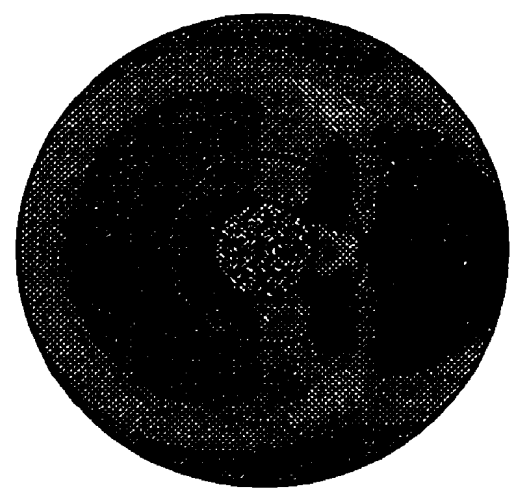

$t=16$

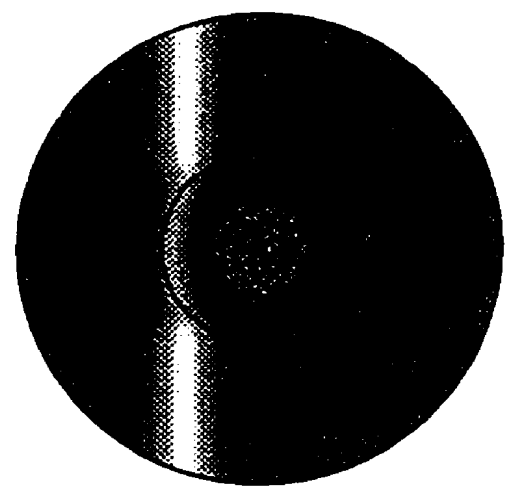

$t=6$

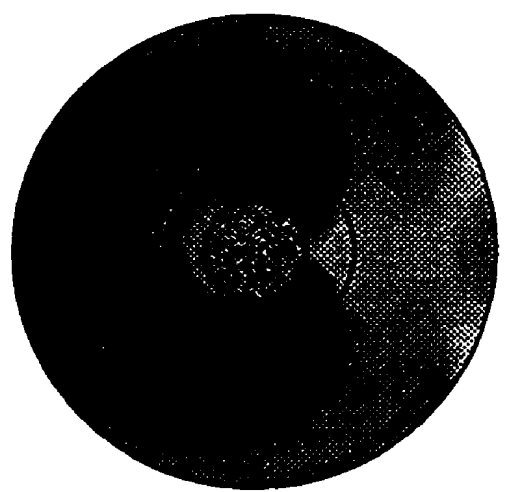

$t=12$

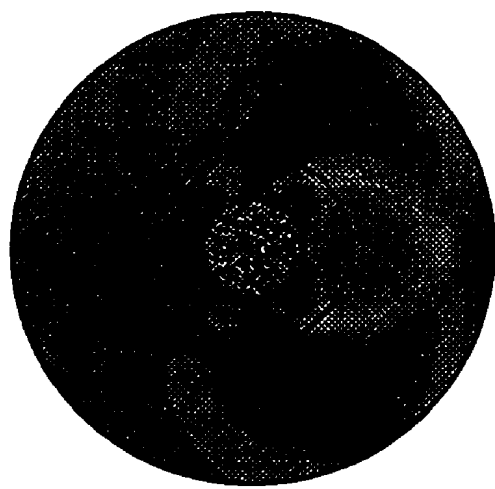

$t=18$

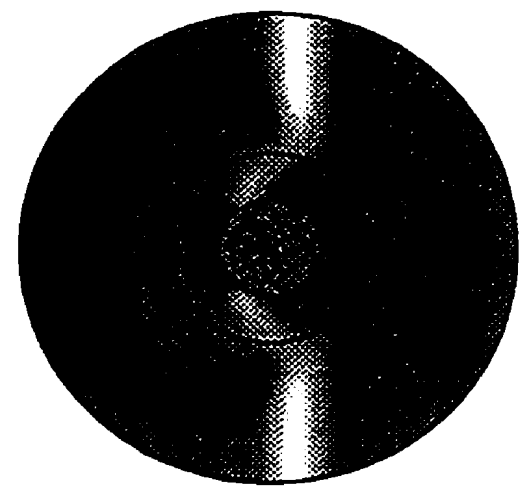

$t=8$

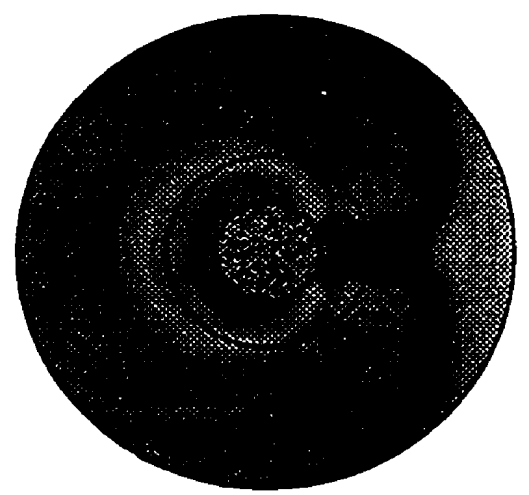

$t=14$

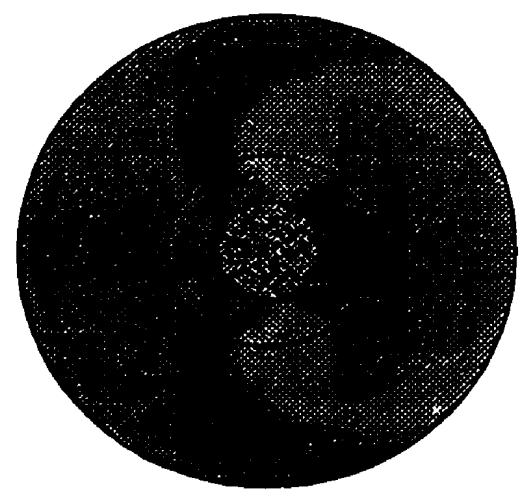

$t=20$

Figure 46: Electric field intensity for Gaussian pulse incident on a circular dielectric scatterer with a perfectly-conducting core

A number of shaded contour plots showing the $z$ component of the electric field intensity, at different times, are shown in Figure 46 . This figure clearly shows the complexity of the wave interactions with the scatterer. The transmission and reflection of the original pulse into and off of the dielectric can be seen at $t=6$ and $t=8$. At $t=8$ through 12 the pulse 
transmitted inside the dielectric reflects off of the perfectly-conducting core and moves out of the domain. By $t=12$ the original pulse has completely moved out of the domain. From $t=14$ through 20 the energy trapped in the dielectric, due to internal reflections, is slowly allowed to escape into free-space. At $t=20$ a significant amount of energy is still present in the dielectric. From a numerical standpoint this simulation is quite successful. The complex physics has been captured using a relatively coarse grid, and the high-accuracy scheme, in conjunction with the interface and boundary conditions, results in a stable and accurate numerical method. 


\section{Conclusions and Recommendations}

\subsection{Conclusions}

A method has been presented for developing high-accuracy finite-difference schemes optimized to provide small dissipation and dispersion errors for a given range of wave frequencies. A number of schemes optimized for different frequency bandwidths are given. Stable numerical boundary schemes that preserve the accuracy of the interior operators are also presented. Two high-accuracy schemes, a maximum-order scheme and a scheme optimized for use with a grid having a resolution of at least $10 \mathrm{PPW}$, have been used to solve the twodimensional time-domain Maxwell equations to simulate electromagnetic wave propagation and scattering. Conditions that can be used at the interface between materials have been presented, and a far-field radiation boundary condition has been implemented which produces very little spurious reflection when used with the high-accuracy schemes.

The schemes have successfully modeled perfectly-conducting and dielectric scatterers on Cartesian and curvilinear grids. The high-accuracy methods have been shown to be more efficient than classical second- and fourth-order methods in terms of both the number of arithmetic operations and the amount of computer memory required. The optimized scheme can produce some error reduction relative to the maximum-order scheme with no additional expense, especially when the number of wavelengths of travel is large. This error reduction is seen even when using a curvilinear grid.

Overall, the results demonstrate that the high-accuracy methods. are an efficient option for simulating the propagation and scattering of high frequency electromagnetic waves. These methods now introduce the possibility of modeling problems that could not be tackled using classical low-order methods and the computing platforms now available. For example, it should now be possible to do radar cross section modeling of an entire aircraft for incident waves having a frequency in the tens of $\mathrm{GHz}$. The application of these high-accuracy methods to problems in other disciplines, such as computational aeroacoustics, is also promising. 


\subsection{Contributions}

The following contributions have been made by this research:

1. A number of high-accuracy finite-difference methods have been developed, including schemes optimized to produce minimum phase and amplitude errors for a given range of frequencies.

2. A solver for the two-dimensional Maxwell equations which uses the new high-accuracy methods has been developed. It is applied to Cartesian and curvilinear grids. Proper material interface conditions, far-field boundary conditions, and stable and accurate numerical boundary schemes have been developed and implemented.

3. Detailed grid resolution studies have demonstrated that the high-accuracy methods are much more efficient than schemes which are second- and fourth-order in space and fourth-order in time. For the problems studied, the high-accuracy methods require 2.5 times less memory and less than half the CPU time required by the fourthorder method used for comparison.

\subsection{Recommendations}

The solver used in this research can model a number of interesting scattering problems, but a more general solver must be written in order to handle a wider range of problems. The new solver should be able to handle arbitrary source configurations, and materials with anisotropic and nonlinear properties (such as materials with frequency-dependent properties). The current solver uses overlapping multi-block grids which have similar topologies. Adding the ability to use grids that have multiple blocks with different topologies is important. The use of such grids will allow problems with very complex geometries to be modeled and can avoid potential problems, such as cylindrical polar singularities. Adding the ability to model subcell structures and improving the handling of regions where field singularities exist are also closely related to grid generation. The new solver should also be extended to handle the full three-dimensional set of Maxwell's equations.

The existing solver can be easily modified to work for other sets of equations describing linear wave propagation and scattering. The acoustic equations and the elastodynamic equations are two sets of equations which describe physics similar to electromagnetic wave 
propagation. To handle scattering problems for these sets of equations, care must be taken to ensure that the proper physical boundary conditions are implemented at material interfaces, and that the proper far-field conditions are imposed.

Further study can be performed on the numerical methods presented in this research. Fourier analysis indicates that compact spatial operators show a lot of promise for modeling wave propagation problems. The simulations performed in the previous chapter should be run using maximum-order and optimized compact schemes, such as the ones given by Haras and Ta'asan [18]. This would determine the relative numerical efficiency of the compact schemes compared to the noncompact schemes. Research should be carried out on stable numerical boundary schemes for use with interior operators of order higher than sixth. The lack of such boundary schemes is a major obstacle in the use of such operators. Also, an attempt at optimizing the numerical boundary conditions and interpolation and extrapolation schemes used at interfaces can be made. It should be possible to tailor the frequency response of these schemes in a way similar to that used on the interior operators. Finally, the Berenger PML absorbing boundary condition should be implemented, and its performance evaluated. 


\section{Appendix A: Obtaining the Strong Conservation Law Form of Equations}

After performing a generalized coordinate transformation on an equation which is in a conservation law form, the new equation is not in strong conservation law form because the grid metrics are located outside the derivatives. This appendix shows how to obtain a strong conservation law form for a two-dimensional equation, see, for example, [1]. During the development, the assumption is made that the grid, and as a result the grid metrics, are not time dependent.

The transformed equation can be written in chain rule form as follows

$$
\partial_{t} \mathbf{Q}+\xi_{x} \partial_{\xi} \mathbf{F}+\eta_{x} \partial_{\eta} \mathbf{F}+\xi_{y} \partial_{\xi} \mathbf{G}+\eta_{y} \partial_{\eta} \mathbf{G}=\mathbf{0}
$$

where, for example, $\partial_{t}=\frac{\partial}{\partial t}$ and $\xi_{x}=\frac{\partial \xi}{\partial x}$. The grid metrics are given by

$$
\xi_{x}=J y_{\eta}, \quad \xi_{y}=-J x_{\eta}, \quad \eta_{x}=-J y_{\xi}, \quad \eta_{y}=J x_{\xi}
$$

where the reciprocal of the Jacobian of the transformation is

$$
J^{-1}=x_{\xi} y_{\eta}-x_{\eta} y_{\xi}
$$

To obtain the strong conservation law form of the equation, first multiply the chain rule form of the equation by the reciprocal of the Jacobian

$$
J^{-1} \partial_{t} \mathbf{Q}+J^{-1} \xi_{x} \partial_{\xi} \mathbf{F}+J^{-1} \eta_{x} \partial_{\eta} \mathbf{F}+J^{-1} \xi_{y} \partial_{\xi} \mathbf{G}+J^{-1} \eta_{y} \partial_{\eta} \mathbf{G}=\mathbf{0}
$$

The terms in front of the derivatives can be forced inside using simple calculus

$$
\begin{array}{r}
{\left[\partial_{1}\left(J^{-1} \mathbf{Q}\right)-\mathbf{Q} \partial_{t} J^{-1}\right]+\left[\partial_{\xi}\left(J^{-1} \xi_{x} \mathbf{F}\right)-\mathbf{F} \partial_{\xi}\left(J^{-1} \xi_{x}\right)\right]+\left[\partial_{\eta}\left(J^{-1} \eta_{x} \mathbf{F}\right)-\mathbf{F} \partial_{\eta}\left(J^{-1} \eta_{x}\right)\right]} \\
+\left[\partial_{\xi}\left(J^{-1} \xi_{y} \mathbf{G}\right)-\mathbf{G} \partial_{\xi}\left(J^{-1} \xi_{y}\right)\right]+\left[\partial_{\eta}\left(J^{-1} \eta_{y} \mathbf{G}\right)-\mathbf{G} \partial_{\eta}\left(J^{-1} \eta_{y}\right)\right]=\mathbf{0}
\end{array}
$$

Recalling that the grid does not change in time, which gives $\partial_{t} J^{-1}=0$, and by rearranging the terms, the following equation is obtained

$$
\begin{array}{r}
\partial_{1}\left(J^{-1} \mathbf{Q}\right)+\partial_{\xi}\left(J^{-1} \xi_{x} \mathbf{F}+J^{-1} \xi_{y} \mathbf{G}\right)+\partial_{\eta}\left(J^{-1} \eta_{x} \mathbf{F}+J^{-1} \eta_{y} \mathbf{G}\right) \\
=\mathbf{F}\left[\partial_{\xi}\left(J^{-1} \xi_{x}\right)+\partial_{\eta}\left(J^{-1} \eta_{x}\right)\right]+\mathbf{G}\left[\partial_{\xi}\left(J^{-1} \xi_{y}\right)+\partial_{\eta}\left(J^{-1} \eta_{y}\right)\right]
\end{array}
$$

The terms in the square brackets on the right hand side of the above equation are called invariants of the transformation. It is very easy to show, by using the relations given in ( A.2 ), that these terms are identically zero. 


$$
\begin{gathered}
\partial_{\xi}\left(J^{-1} \xi_{x}\right)+\partial_{\eta}\left(J^{-1} \eta_{x}\right)=\partial_{\xi} y_{\eta}-\partial_{\eta} y_{\xi}=y_{\eta \xi}-y_{\xi \eta}=0 \\
\partial_{\xi}\left(J^{-1} \xi_{x}\right)+\partial_{\eta}\left(J^{-1} \eta_{x}\right)=-\partial_{\xi} x_{\eta}+\partial_{\eta} x_{\xi}=-x_{\eta \xi}+x_{\xi \eta}=0
\end{gathered}
$$

The above results are obtained by using the fact that the derivative operators are commutative, i.e. $\frac{\partial^{2}}{\partial \xi \partial \eta}=\frac{\partial^{2}}{\partial \eta \partial \xi}$.

Noting that the right hand side of ( A.6) is zero, the strong conservation law form of the equation is now obtained

$$
\partial_{t} \hat{\mathbf{Q}}+\partial_{\xi} \hat{\mathbf{F}}+\partial_{\eta} \hat{\mathbf{G}}=\mathbf{0}
$$

where

$$
\hat{\mathbf{Q}}=J^{-1} \mathbf{Q}, \quad \hat{\mathbf{F}}=J^{-1}\left(\xi_{x} \mathbf{F}+\xi_{y} \mathbf{G}\right), \quad \hat{\mathbf{G}}=J^{-1}\left(\eta_{x} \mathbf{F}+\eta_{y} \mathbf{G}\right)
$$




\section{Appendix B: Artificial Source Terms}

Problems can occur when numerical approximations are used for the spatial derivatives of an equation that is expressed in a generalized coordinate system and is written in a strong conservation law form [1]. If the operators used for the approximations are not formulated correctly, the solution will become polluted by artificial source terms. These sources have no physical counterparts, because they are an artifact of the numerics not being able to properly model the metric invariants discussed in Appendix A. The problem stems from the spatial operators not being commutative over the entire grid. Difficulties usually occur near interfaces and boundaries where characteristic forms of the equations are used in conjunction with upwind and upwind-biased numerical boundary schemes. An easy way to test for the sources is to set the field values in the numerical domain to be a non-zero constant, and then to determine the approximation for the field's time derivatives. If the result is not zero, and barring any other errors, the source terms are present.

Consider the following invariant of the grid transformation

$$
\frac{\partial}{\partial \xi}\left(\frac{1}{J} \frac{\partial \xi}{\partial x}\right)+\frac{\partial}{\partial \eta}\left(\frac{1}{J} \frac{\partial \eta}{\partial x}\right)
$$

This term evaluates to zero analytically, but problems arise when the analytical derivatives are replaced with numerical approximations. This term will not evaluate to zero if the approximations have not been specially formulated. By inspecting ( A.6), it easy to see that the following source term, which is an artifact of the numerical methods, will be present

$$
\mathbf{F}\left[\frac{\partial}{\partial \xi}\left(\frac{1}{J} \frac{\partial \xi}{\partial x}\right)+\frac{\partial}{\partial \eta}\left(\frac{1}{J} \frac{\partial \eta}{\partial x}\right)\right]
$$

There is a similar term which is proportional to $\mathbf{G}$.

When considering Maxwell's equations, it is clear that the artificial source terms are proportional to the field variables multiplied by terms which are dependent on the grid. This leads to an easy method for correcting these errors. Since the grids being considered are not dependent on time, a correction factor can be determined at the beginning of a simulation. By inspecting the characteristic splitting given by equations ( 2.47$)$ and (2.48), it is clear that the source term errors at a node can be written as follows 


$$
\mathbf{e}=D_{z}\left[\begin{array}{ccc}
c & 0 & 0 \\
0 & \frac{1}{c} & 0 \\
0 & 0 & \frac{1}{c}
\end{array}\right] \mathbf{e}_{1}+B_{x}\left[\begin{array}{ccc}
\frac{1}{\mu} & 0 & 0 \\
0 & c & 0 \\
0 & 0 & c
\end{array}\right] \mathbf{e}_{2}+B_{y}\left[\begin{array}{ccc}
\frac{1}{\mu} & 0 & 0 \\
0 & c & 0 \\
0 & 0 & c
\end{array}\right] \mathbf{e}_{3}
$$

where the $e_{i}$ are error vectors which depend only on the grid. These error vectors can be found using the following procedure:

1. Set the material properties in the numerical domain to constants.

2. Set $D_{z}=1, B_{x}=B_{y}=0$; and calculate $\mathbf{e}_{1}=\left[\begin{array}{ccc}\frac{1}{c} & 0 & 0 \\ 0 & \varepsilon & 0 \\ 0 & 0 & \varepsilon\end{array}\right] \delta, \hat{\mathbf{Q}}$.

3. Set $B_{x}=1, D_{z}=B_{y}=0$; and calculate $\mathbf{e}_{2}=\left[\begin{array}{ccc}\mu & 0 & 0 \\ 0 & \frac{1}{c} & 0 \\ 0 & 0 & \frac{1}{c}\end{array}\right] \delta, \hat{\mathbf{Q}}$.

4. Set $B_{y}=1, D_{z}=B_{x}=0$; and calculate $\mathbf{e}_{3}=\left[\begin{array}{ccc}\mu & 0 & 0 \\ 0 & \frac{1}{c} & 0 \\ 0 & 0 & \frac{1}{c}\end{array}\right] \delta_{t} \hat{\mathbf{Q}}$.

$\delta_{t} \hat{\mathbf{Q}}$ represents the numerical approximation to the time derivative of the field values which is calculated using equation ( 3.13 ). To generate the corrected approximation to the time derivative, the following equation is used

$$
\frac{\partial \hat{\mathbf{Q}}}{\partial t} \approx-\left(\delta_{\xi}^{a} \hat{\mathbf{F}}+\delta_{\xi}^{s}|\hat{\mathbf{F}}|\right)-\left(\delta_{\eta}^{a} \hat{\mathbf{G}}+\delta_{\eta}^{s} \mid \hat{\mathbf{G}}\right)-\mathbf{e}
$$

The use of this correction factor will have very little impact on the total number of operations in the simulation, but it will have a significant impact on the amount of memory required. The three error vectors must be stored at every node in the numerical domain, resulting in the need for nine additional storage locations per node. 


\section{Appendix C: Coefficients of Optimized Finite-Difference Schemes}

The following table presents the coefficients of the schemes optimized for a grid having a minimum resolution given in terms of the number of points per wavelength (PPW).

\begin{tabular}{|c|c|c|c|}
\hline Minimum PPW & 10 & 7.5 & 5 \\
\hline \hline$a_{1}$ & 0.759961 & 0.767920 & 0.791568 \\
\hline$a_{2}$ & -0.158122 & -0.164845 & -0.186214 \\
\hline$a_{3}$ & 0.0187609 & 0.0205901 & 0.0269529 \\
\hline \hline$d_{0}$ & 0.1 & 0.1 & 0.1 \\
\hline$d_{1}$ & -0.0763846 & -0.0774664 & -0.0804523 \\
\hline$d_{2}$ & 0.0322896 & 0.0341999 & 0.0403545 \\
\hline$d_{3}$ & -0.00590500 & -0.00673357 & -0.00990220 \\
\hline$\alpha_{1}$ & 0.168850 & 0.173113 & 0.176095 \\
\hline$\alpha_{2}$ & 0.197348 & 0.193146 & 0.188949 \\
\hline$\alpha_{3}$ & 0.250038 & 0.250218 & 0.250642 \\
\hline$\alpha_{4}$ & 0.333306 & 0.333135 & 0.332852 \\
\hline$\alpha_{5}$ & 0.5 & 0.5 & 0.5 \\
\hline
\end{tabular}

Table 5: Coefficients of optimized finite-difference schemes 


\section{Appendix D: Dielectric Square - Interpolation and Corners}

The following fifth-order polynomial interpolation schemes are used, when necessary, to obtain the fields on the interface of the dielectric square

$$
\begin{gathered}
u_{i+1 / 2}=\left(3 u_{i-2}-25 u_{i-1}+150 u_{i}+150 u_{i+1}-25 u_{i+2}+3 u_{i+3}\right) / 256 \\
u_{i+1 / 2}=\left(-7 u_{i-1}+105 u_{i}+210 u_{i+1}-70 u_{i+2}+21 u_{i+3}-3 u_{i+4}\right) / 256 \\
u_{i+1 / 2}=\left(63 u_{i}+315 u_{i+1}-210 u_{i+2}+126 u_{i+3}-45 u_{i+4}+7 u_{i+5}\right) / 256 \\
u_{i}=\frac{3}{4}\left(u_{i-1}+u_{i+1}\right)-\frac{3}{10}\left(u_{i-2}+u_{i+2}\right)+\frac{1}{20}\left(u_{i-3}+u_{i+3}\right)
\end{gathered}
$$

Figure 47 shows a section of the domain near one of the corners of the dielectric square. The outgoing characteristic variables, which are obtained along the left-hand interface in each domain, as discussed in $\S 4.1 .2$, are denoted by $w_{+}$and $w .$. At the nodes along the outside of the interface marked with a $*$, the $w_{+}$characteristic variable is determined from interpolated fields. These fields are obtained using the values from the surrounding interface nodes and the schemes given in (D.1), (D.2), and (D.3). The non-centered interpolation schemes are only used at nodes near the corners of the dielectric. After this interpolation is performed, $D_{z}$ and $B_{t}$ can be found at all nodes along both sides of the interface, excluding the corner nodes.

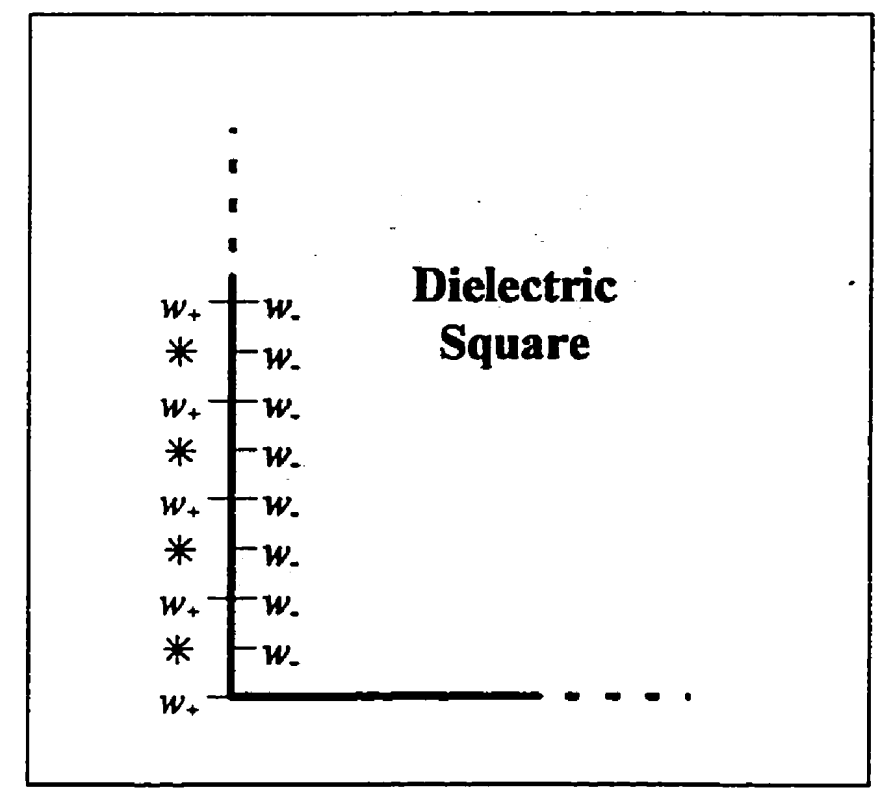

Figure 47: Nodes, $*$, on outside surface of dielectric at which $w_{+}$must be interpolated 
To determine the normal component of the magnetic flux density, which is continuous across the interface, different extrapolation schemes must be used depending on whether or not gridlines from the interior and exterior of the dielectric meet at the interface. As shown in Figure $48 \mathrm{a}$ ), the value of $B_{n}$ at a node which is part of the interior and exterior of the domain, marked with an 0 , is found by extrapolating the values from both sides of the interface, found at the nodes marked by an $x$, using a fifth-order polynomial method and then averaging these values. Figure 48 b) shows all the data needed to obtain $B_{n}$ at a node on the interface which is only present inside the dielectric square, again marked by an 0 . The value on the interface is obtained, as discussed above, by extrapolating the data found at the locations marked by an $\mathrm{X}$. Because there are no nodes at the $x$ locations uniside the dielectric, (D.1) is used to interpolate the data found at the nodes marked by $a *$. Using these methods às an example, $B_{n}$ can be found at all the nodes along the interface, excluding the corners.

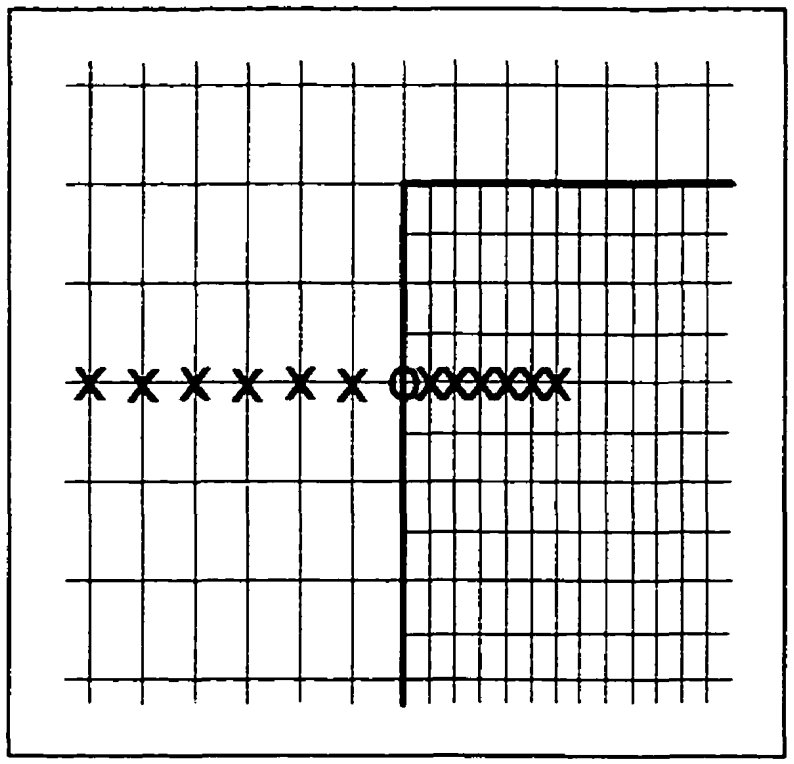

a)

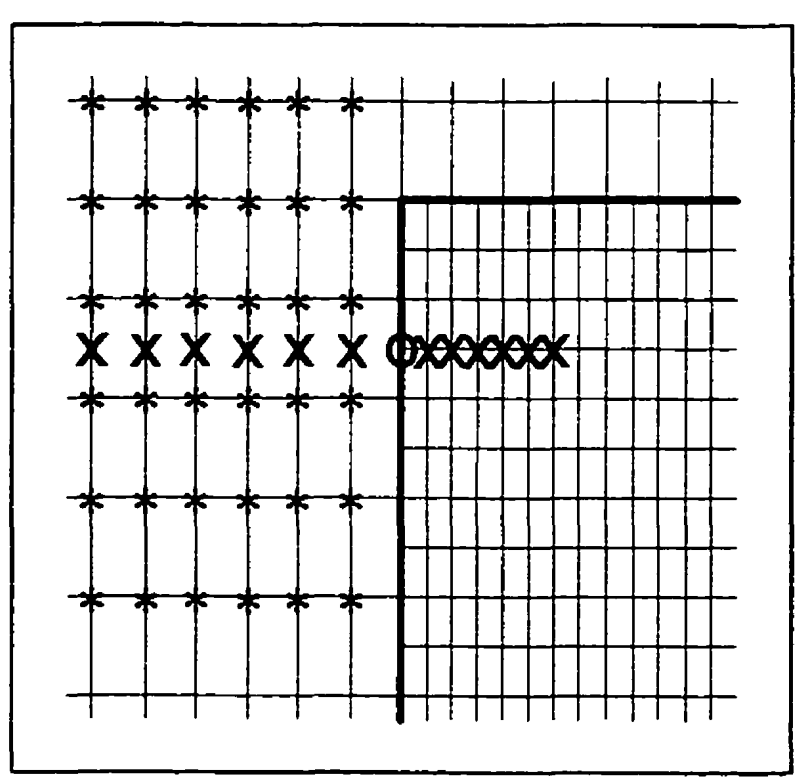

b)

Figure 48: Data needed to interpolate $B_{n}$ onto the interface for a) a node located on both sides of the interface and b) a node located only on the dielectric side of the interface

As mentioned above, the corners must be handled as special cases. It is only necessary to store the field values at the corner nodes for the free space part of the domain. The corner values in the interior of the dielectric are never used during the simulation because they are not needed when finding the spatial derivatives of the field values at nodes near the corners. The fields at the corners, outside the dielectric, are obtained by taking the average value of the 
results of interpolating the data found along the $x$ and $y$ grid lines which intersect each corner node. The fifth-order polynomial interpolation scheme, (D.4), is used along these grid lines. 


\section{Appendix E: Analytical Solution for a Harmonic Electromagnetic Wave Incident on a Perfectly-Conducting Circular Cylinder}

Many texts present the analytical solution for a harmonic electromagnetic wave incident on a perfectly-conducting circular cylinder (see, for example, [19]). Consider the system shown in Figure 49, where a $z$-polarized (Transverse Magnetic) wave is incident upon a perfectly-conducting cylinder of radius, $a$. The frequency domain $z$-component of the incident electric field intensity, represented as a complex number, is given by:

$$
\mathrm{E}_{z}^{i}=E_{0} e^{-j i x}=E_{0} e^{-j p \rho \cos \varphi}
$$

where $j=\sqrt{-1}$.

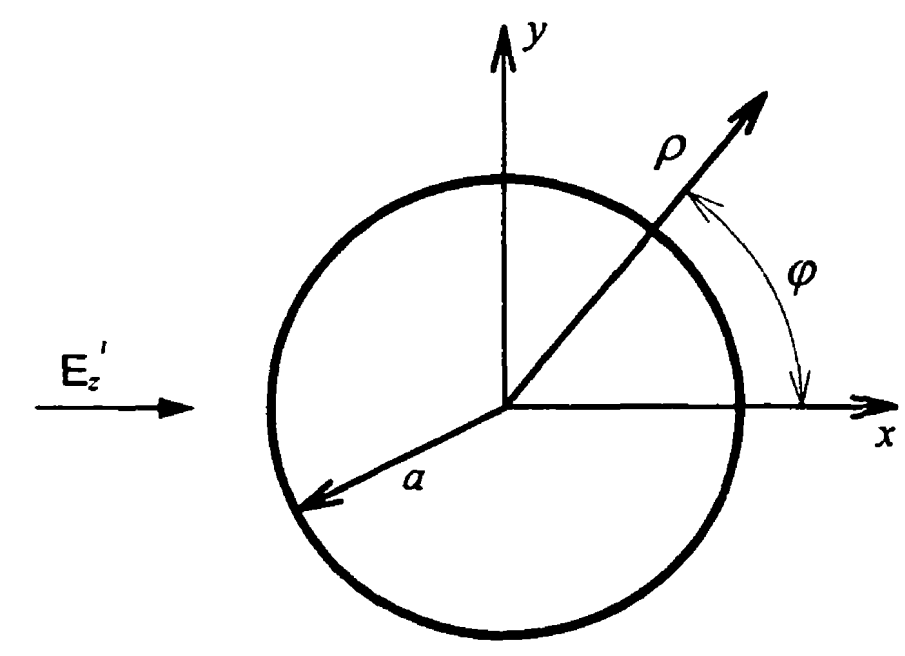

Figure 49: TM harmonic wave incident on a perfectly-conducting circular cylinder of radius $a$

The resulting scattered electric field intensity is given by:

$$
\mathrm{E}_{:}^{s}=E_{0} \sum_{n=-\infty}^{\infty} j^{-n} a_{n} \mathrm{H}_{n}^{(2)}(\kappa \rho) e^{j m \rho}
$$

where $a_{n}=-\frac{\mathrm{J}_{n}(\kappa a)}{\mathrm{H}_{n}^{(2)}(\kappa a)}, \mathrm{J}_{n}$ is Bessel's function of the first kind, and $\mathrm{H}_{n}^{(2)}$ is Hankel's function of the second kind. The total field is found by summing the incident and scattered fields. The following relations are used to find the magnetic field intensities: 


$$
\begin{aligned}
& \mathrm{H}_{\rho}=\frac{j}{\omega \mu} \frac{1}{\rho} \frac{\partial \mathrm{E}_{z}}{\partial \varphi} \\
& H_{\odot}=-\frac{j}{\omega \mu} \frac{\partial \mathrm{E}_{z}}{\partial \rho}
\end{aligned}
$$

where $\omega$ is the temporal angular frequency of the wave, and $\mu$ is the magnetic permeability. The $x$ and $y$ components of the magnetic field intensities are:

$$
\begin{aligned}
& H_{x}=H_{p} \cos \varphi-H_{\varphi} \sin \varphi \\
& H_{y}=H_{\rho} \sin \varphi+H_{p} \cos \varphi
\end{aligned}
$$

To obtain the field values in the time domain the following relations are used:

$$
\begin{aligned}
& E_{x}=\operatorname{Re}\left(\mathrm{E}_{z} e^{j \alpha x}\right) \\
& H_{x}=\operatorname{Re}\left(\mathrm{H}_{x} e^{j \alpha x}\right) \\
& H_{y}=\operatorname{Re}\left(\mathrm{H}_{y} e^{j \alpha x}\right)
\end{aligned}
$$




\section{References}

1. D.A. Anderson, J.C. Tannehill, and R.H. Pletcher, Computational Fluid Mechanics and Heat Transfer, Hemisphere, New York, 1984.

2. A. Bayliss, and E. Turkel, Radiation boundary conditions for wave-like equations, Communications on Pure and Applied Mathematics, Vol. 33, 1980, pp. 707-725.

3. J.P. Berenger, A perfectly matched layer for the absorption of electromagnetics wave, Journal of Computational Physics, Vol. 114, 1994, pp. 185-200.

4. D.G. Bishop, and D.A. Anderson, A comparison of finite-volume time-domain schemes for the Macwell equations, AIAA Paper 92-0456, 1992.

5. A.C. Cangellaris, C.-C. Lin, and K.K. Mei, Point-matched time-domain finite element methods for electromagnetic radiation and scattering, IEEE Transactions on Antennas and Propagation, Vol. 35, 1987, pp. 1160-1173.

6. M.H. Carpenter, D. Gottlieb, and S. Abarbanel, Time-stable boundary conditions for finite-difference schemes solving hyperbolic systems: methodology and application to high-order compact schemes, Journal of Computational Physics, Vol. 111, 1994, pp. 220-236.

7. J.E. Castillo, J.M. Hyman, M.J. Shashkov, and S. Steinberg, High-order mimetic finite difference methods on nonuniform grids, in Proceedings of the Third International Conference on Spectral and Bigh Order Methods, Houston Journal of Mathematics, Houston, Texas, 1995, pp. 347-361.

8. W.C. Chew, and W.H. Weedon, $A 3 D$ perfectly matched medium from modified Maxwell's equations with stretched coordinates, Microwave and Optical Technology Letters, Vol. 7, 1994, pp. 599-604.

9. G. Cohen, and P. Joly, Fourth order schemes for the heterogeneous acoustics equation, Computational Methods in Applied Mechanics and Engineering, Vol. 80, 1990, pp. 397-407.

10. M.A. Dablain, The application of high-order differencing to the scalar wave equation, Geophysics, Vol. 51, 1986, pp. 54-66.

11. T. Deveze, L. Beaulie, and W. Tabbara, A fourth order scheme for the FDTD algorithm applied to Maxwell equations, in IEEE Antennas and Propagation Society International Symposium Digest, Chicago, Illinois, 1992, pp. 342-345.

12. B. Engquist, and A. Majda, Absorbing boundary conditions for the numerical simulation of waves, Mathematics of Computation, Vol. 31, 1977, pp. 629-651.

13. M. Feliziani, and F. Maradei, Field-to-wire coupling using the finite element-time domain (FE-TD) method, IEEE Transactions on Magnetics, Vol. 31, 1995, pp. 1586-1589.

14. M.A. Fusco, M.V. Smith, and L.W. Gordon, A three-dimensional FDTD algorithm in curvilinear coordinates, IEEE Transactions on Antennas and Propagation, Vol. 39, 1991, pp. 1463-1471.

15. D. Gaitonde, and J.S. Shang, High-order finite-volume schemes in wave propagation phenomena, AIAA Paper 96-2335, 1996.

16. M.B. Giles, Nonreflecting boundary conditions for Euler equation calculations, AIAA Journal, Vol. 28, 1990 , pp. 2050-2058.

17. B. Gustafsson, H.-O. Kreiss, and A. Sundström, Stability theory of difference approximations for mixed initial boundary value problems. II, Mathematics of Computation. Vol. 26, 1972, pp. 649-686. 
18. Z. Haras, and S. Ta'asan, Finite difference schemes for long-time integration, Journal of Computational Physics, Vol. 114, 1994, pp. 265-279.

19. R.F. Harrington, Time-Harmonic Electromagnetic Fields, McGraw-Hill, New York, 1961.

20. R. Harrington, Field Computation by Moment Methods, Reprint, Krieger, Malabar, Florida, 1982.

21. W.H. Hayt, Jr., Engineering Electromagnetics, Fourth Edition, McGraw-Hill, New York, 1981.

22. R.L. Higdon, Numerical absorbing boundary conditions for the wave equation, Mathematics of Computation, Vol. 49, 1987, pp. 65-90.

23. H.H. Hinken, Conducting spheres in rectangular waveguides, IEEE Transactions on Microwave Theory and Techniques, Vol. 28, 1980, pp. 711-714.

24. C. Hirsch, Numerical Computation of Internal and External Flows, Vol. 2: Computational Methods for Inviscid and Viscous Flows, Wiley, England, 1990.

25. R. Hixon, S.-H. Shih, and R.R. Mankbadi, Evaluation of boundary conditions for computational aeroacoustics, AIAA Journal, Vol. 33, 1995, pp. 2006-2012.

26. O. Holberg, Computational aspects of the choice of operator and sampling interval for numerical differentiation in large-scale simulation of wave phenomena, Geophysical Prospecting, Vol. 35, 1987, pp. 629-655.

27. R. Holland, Finite difference solutions of Maxwell's equations in generalized nonorthogonal coordinates, IEEE Transaction on Nuclear Science, Vol. 30, 1983, pp. 4589-4591.

28. F.Q. Hu, M.Y. Hussaini, and J.L. Manthey, Low-dissipation and low-dispersion Runge-Kutta schemes for computational acoustics, Journal of Computational Physics, Vol. 124, 1996, pp. 177-191.

29. H.M. Jurgens, and D.W. Zingg. Implementation of a high-accuracy finite-difference scheme for linear wave phenomena, in Proceedings of the Third International Conference on Spectral and High Order Methods, Houston Journal of Mathematics, Houston, Texas, 1995, pp. 363-372.

30. H.M. Jurgens, and D.W. Zingg, Numerical solution of the time-domain Maxwell equations using highaccuracy finite-difference methods, submitted to Journal of Computational Physics, June 1996.

31. T.G. Jurgens, A. Taflove, K. Umashankar, and T.G. Moore, Finite-difference time-domain modeling of curved surfaces, IEEE Transactions on Antennas and Propagation, Vol. 40, 1992, pp. 357-366.

32. D.S. Katz, E.T. Thiele, and A. Taflove, Validation and extension to three dimensions of the Berenger PML absorbing boundary condition for FD-TD meshes, IEEE Microwave and Guided Wave Letters, Vol. 4, 1994, pp. 268-270.

33. J.B. Keller, Geometrical theory of diffraction, Journal of the Optical Society of America, Vol. 52, 1962, pp. 116-130.

34. J.W. Kim, and D.J. Lee, Optimized compact finite difference schemes with maximum resolution, AlAA Journal, Vol. 34, 1996, pp. 887-893.

35. M. Kindelan, A. Kamel, and P. Sguazzero, On the construction and efficiency of staggered numerical differentiators for the wave equation, Geophysics, Vol. 55, 1990, pp. 107-110.

36. A. Konrad, and P. Silvester, Triangular finite elements for the generalized Bessel equation of order $m$, International Journal for Numerical Methods in Engineering, Vol. 7, 1973, pp. 43-55.

37. Z. Kopal, Numerical Analysis, $2^{\text {nd }}$ Edition, John Wiley and Sons, New York, 1961. 
38. H.-O. Kreiss, and $\mathrm{L}$. Wu, On the stability definitions of difference approximations for the initial boundary value problem, Applied Numerical Mathematics, Vol. 12, 1993, pp. 213-227.

39. G.A. Kriegsmann, A. Taflove, and K.R. Umashankar, A new formulation of electromagnetic wave scattering using an on-surface radiation boundary condition approach. IEEE Transactions on Antennas and Propagation, Vol. 35, 1987, pp. 153-161.

40. L. Lapidus and J. Seinfeld, Numerical Solution of Ordinary Differential Equations, Academic Press, New York, 1971.

41. J.D. Lawson, An order five Runge-Kutta process with extended region of stability, SLAM Journal on Numerical Analysis, Vol. 3, 1966, pp. 593-597.

42. S.K. Lele, Compact finite difference schemes with spectral-like resolution, Journal of Computational Physics, Vol. 103, 1992, pp. 16-42.

43. Y. Liu, Fourier analysis of numerical algorithms for the Maxwell equations, Journal of Computational Physics, Vol. 124, 1996, pp. 396-416.

44. D.P. Lockard, K.S. Brentner, and H.L. Atkins, High accuracy algorithms for computational aeroacoustics, ALAA Paper 94-0460, 1994.

45. N.K. Madsen, and RW. Ziolkowski, A three-dimensional modified finite volume technique for Maxwell's equations, Electromagnetics, Vol. 10, 1990, pp. 147-161.

46. N.K. Madsen, Divergence preserving discrete surface integral methods for Maxwell's curl equations using non-orthogonal unstructured grids, Journal of Computational Physics, Vol. 119, 1995, pp. 34-45.

47. A.H. Mohammadian, V. Shankar, and W.F. Hall, Computation of electromagnetic scattering and radiation using a time-domain finite-volume discretization procedure, Computer Physics Communications, Vol. 68, 1991, pp. 262-281.

48. T.G. Moore, J.G. Blaschak, A. Taflove, and G.A. Kriegsmann, Theory and application of radiation boundary operators, invited review paper, IEEE Transactions on Antennas and Propagation, Vol. 36, 1988, pp. 1797-1812.

49. G. Mur, Absorbing boundary conditions for the finite-difference approximation of the time-domain electromagnetic-field equations, IEEE Transactions on Electromagnetic Compatibility, Vol. 23, 1981, pp. 377-382.

50. E.A. Navarro, C. Wu, P.Y. Chung, and J. Litva, Application of PML superabsorbing boundary condition to non-orthogonal FDTD method, Electronics Letters, Vol. 30, 1994, pp. 1654-1656.

51. R.W. Noack, and D.A. Anderson, Time domain solutions of Maxwell's equations using a finite-volume formulation, AIAA Paper 92-0451, 1992.

52. P. Olsson, Summation by Parts, Projections, and Stability I, Mathematics of Computation, Vol. 64, 1995, pp. 1035-1065.

53. B. Petitjean, R. Löhner, and C.R. DeVore, Finite element solvers for radar cross-section (RCS) calculations, AIAA Paper 92-0455, 1992.

54. T.K. Sarkar, X. Yang, and E. Arvas, Limited survey of various conjugate gradient methods for solving complex matrix equations arising in electromagnetic wave interaction, Wave Motion, Vol. 10, 1988, pp. 527-546. 
55. P. Sguazzero, M. Kindelan, and A. Kamel, Dispersion-bounded numerical integration of the elastodynamic equations with cost-effective staggered schemes, Computer Methods in Applied Mechanics and Engineering, Vol. 80, 1990, pp. 165-172.

56. J.S. Shang, and R. M. Fithen, A comparative study of characteristic-based algorithms for the Maxwell equations, Journal of Computational Physics, Vol. 125, 1996, pp. 378-394.

57. J.S. Shang, and D. Gaitonde, On high resolution schemes for time-dependent Maxwell equations, AIAA Paper 96-0832, 1996.

58. J.S. Shang, D. Gaitonde, and K. Wurtzler, Scattering simulations of computational electromagnetics, AIAA Paper 96-2337, 1996.

59. V. Shankar, W.F. Hall, and A.H. Mohammadian, $A$ time-domain differential solver for electromagnetic scattering problems, Proceedings of the IEEE, Vol. 77, No. 5, 1989, pp. 709-72 I.

60. V. Shankar, W.F. Hall, and A.H. Mohammadian, $A$ three-dimensional Maxwell's equation solver for computation of scattering from layered media, IEEE Transactions on Magnetics, Vol. 25, 1989, pp. 3098-3103.

61. V. Shankar, W.F. Hall, and A.H. Mohammadian, $A$ CFD-based finite-volume procedure for computational electromagnetics - Interdisciplinary applications of CFD methods, AIAA Paper 89-1987CP, 1989.

62. V. Shankar, A.H. Mohammadian, and W.F. Hall, A time-domain finite-volume treatment for the Maxwell equations, Electromagnetics, Vol. 10, 1990, pp. 127-145.

63. V. Shankar, W.F. Hall, A. Mohammadian, and C. Rowell, Algorithmic aspects and supercomputing trends in computational electromagnetics, AIAA Paper 93-0367, 1993.

64. K.L. Shlager, and J.B. Schneider, $A$ selective survey of the finite-difference time-domain literature, IEEE Antennas and Propagation Magazine, Vol. 37, 1995, pp. 39-57.

65. P. Silvester, High-order polynomial triangular finite elements for potential problems, International Journal of Engineering Science, Vol. 7, 1969, pp. 849-861.

66. P. Silvester, Tetrahedral polynomial finite elements for the Helmholtz equation, International Journal for Numerical Methods in Engineering, Vol. 4, 1972, pp. 405-413.

67. P. Silvester, Construction of triangular finite element universal matrices, International Journal for Numerical Methods in Engineering. Vol. 12, 1978, pp. 237-244.

68. P.P. Silvester, and R.L. Ferrari, Finite Elements for Electrical Engineers, Second Edition, Reprint, Cambridge University Press, 1991.

69. B. Strand, High-order finite difference methods for hyperbolic IBVP, in Proceedings of the Third International Conference on Spectral and High Order Methods, Houston Journal of Mathematics, Houston, Texas, 1995, pp. 409-420.

70. A. Taflove, K.R. Umashankar, B. Beker, F. Harfoush, and K.S. Yee, Detailed FD-TD analysis of electromagnetic fields penetrating narrow slots and lapped joints in thick conducting screens, IEEE Transactions on Antennas and Propagation, Vol. 36, 1988, pp. 247-257.

71. A. Taflove, Re-inventing electromagnetics: supercomputing solution of Maxwell's equations via direct time integration on space grids, AIAA Paper 92-0333, 1992.

72. A. Taflove, Computational Electrodynamics: The Finite-Difference Time-Domain Method, Artech House, Boston, 1995. 
73. C.K. Tam, and J.C. Webb, Dispersion-relation-preserving finite difference schemes for computational acoustics, Journal of Computational Physics, Vol. 107, 1993, pp. 262-281.

74. J.P. Thomas, and P.L. Roe, Development of non-dissipative numerical schemes for computational aeroacoustics, AIAA Paper 93-3382, 1993.

75. K.W. Thompson, Time-dependent boundary conditions for hyperbolic systems, Journal of Computational Physics, Vol. 68, 1987, pp. 1-24.

76. K.W. Thompson, Time-dependent boundary conditions for hyperbolic systems, II, Journal of Computational Physics, Vol. 89, 1990, pp. 439-461.

77. K.R. Umashankar, A. Taflove, and B. Beker, Calculation and experimental validation of induced currents on coupled wires in an arbitrary shaped covity, IEEE Transactions on Antennas and Propagation, AP-35, 1987, pp. 1248-1257.

78. K. Umashankar, and A. Taflove, Computational Electromagnetics, Artech House, Boston, 1993.

79. R. Vichnevetsky, and F. De Schutter, $A$ frequency analysis of finite difference and finite-element methods for initial value problems, in Advances in Computer Methods for Partial Differential Equations, R. Vichnevetsky, ed., AICA/MMACS, Rutgers University, NJ, 1975, pp. 46-52.

80. M. Vinokur, and M. Yarrow, Finite-surface method for the Moxwell equations in generalized coordinates, AIAA Paper 93-0463, 1993.

81. M. Vinokur, and M. Yarrow, Finite-surface method for the Maxwell equations with corner singularities, AIAA Paper 94-0233, 1994.

82. C. Wu, E.A. Navarro, P.Y. Chung, and J. Litva, Modeling of waveguide structures using the nonorthogonal FDTD method with a PML absorbing boundary, Microwave and Optical Technology Letters, Vol. 8, 1995, pp. 226-228.

83. M. Yarrow, J.A. Vastano, and H. Lomax, Pulsed plane wave analytic solutions for generic shapes and the validation of Maxwell's equations solvers, AIAA Paper 92-0016, 1992.

84. K.S. Yee, Numerical solution of initial boundary value problems involving Maxwell's equations in isotropic media, IEEE Transactions on Antennas and Propagation, Vol. 14, 1966, pp. 302-307.

85. K.S. Yee, J.S. Chen, and A.H. Chang, Conformal finite-difference time-domain (FDTD) with overlapping grids, IEEE Transactions on Antennas and Propagation, Vol. 40, 1992, pp. 1068-1075.

86. K.S. Yee, and J.S. Chen, Conformal hybrid finite difference time domain and finite volume time domain, IEEE Transactions on Antennas and Propagation, Vol. 42, 1994, pp. 1450-1455.

87. S.-T. Yu, L.S. Hultgren, and N.-S. Liu, Direct calculations of waves in fuid flows using high-order compact difference scheme, AlAA Paper 93-0148, 1993.

88. D.W. Zingg, and H. Lomax, On the eigensystems associated with numerical boundary schemes for hyperbolic equations, in Numerical Methods for Fluid Dynamics, M.J. Baines, and K.W. Morton, eds., Clarendon Press, Oxford, 1993, pp. 473-481.

89. D.W. Zingg, H. Lomax, and H.M. Jurgens, An optimized finite-difference scheme for wave propagation problems, AIAA Paper 93-0459, 1993.

90. D.W. Zingg, and H. Lomax, Some aspects of high-order numerical solutions of the linear convection equation with forced boundary conditions, AIAA Paper 93-3381, in Proceedings of the $11^{\text {th }}$ AIAA Computational Fluid Dynamics Conference, American Institute of Aeronautics and Astronautics, New York, 1993. 
91. D.W. Zingg, P.D. Giansante, and H.M. Jurgens, Experiments with high-accuracy finite-difference schemes for the time-domain Maxwell equations, AIAA Paper 94-0232, 1994.

92. D.W. Zingg, E.M. Epstein, and H.M. Jurgens, $A$ comparison of finite-difference schemes for computational aeroacoustics, ALAA Paper 95-0162, 1995.

93. D.W. Zingg, and T.T. Chisholm, Runge-Kutta methods for linear problems, AIAA Paper 95-1756, 1995.

94. D.W. Zingg, On finite-difference methods for simulating linear acoustic wave propagation, AIAA Paper 96-0280, 1996.

95. D.W. Zingg, H. Lomax, and H.M. Jurgens, High-accuracy finite-difference schemes for linear wave propagation, SIAM Journal on Scientific Computing, Vol. 17, 1996, pp. 328-346.

96. S.S. Zivanovic, K.S. Yee, and K.K. Mei, $A$ subgridding method for the time-domain finite-difference method to solve Maxwell's equations, IEEE Transactions on Microwave Theory and Techniques, Vol. 39, 1991, pp. 471-479. 


\section{TEST TARGET (QA-3)}
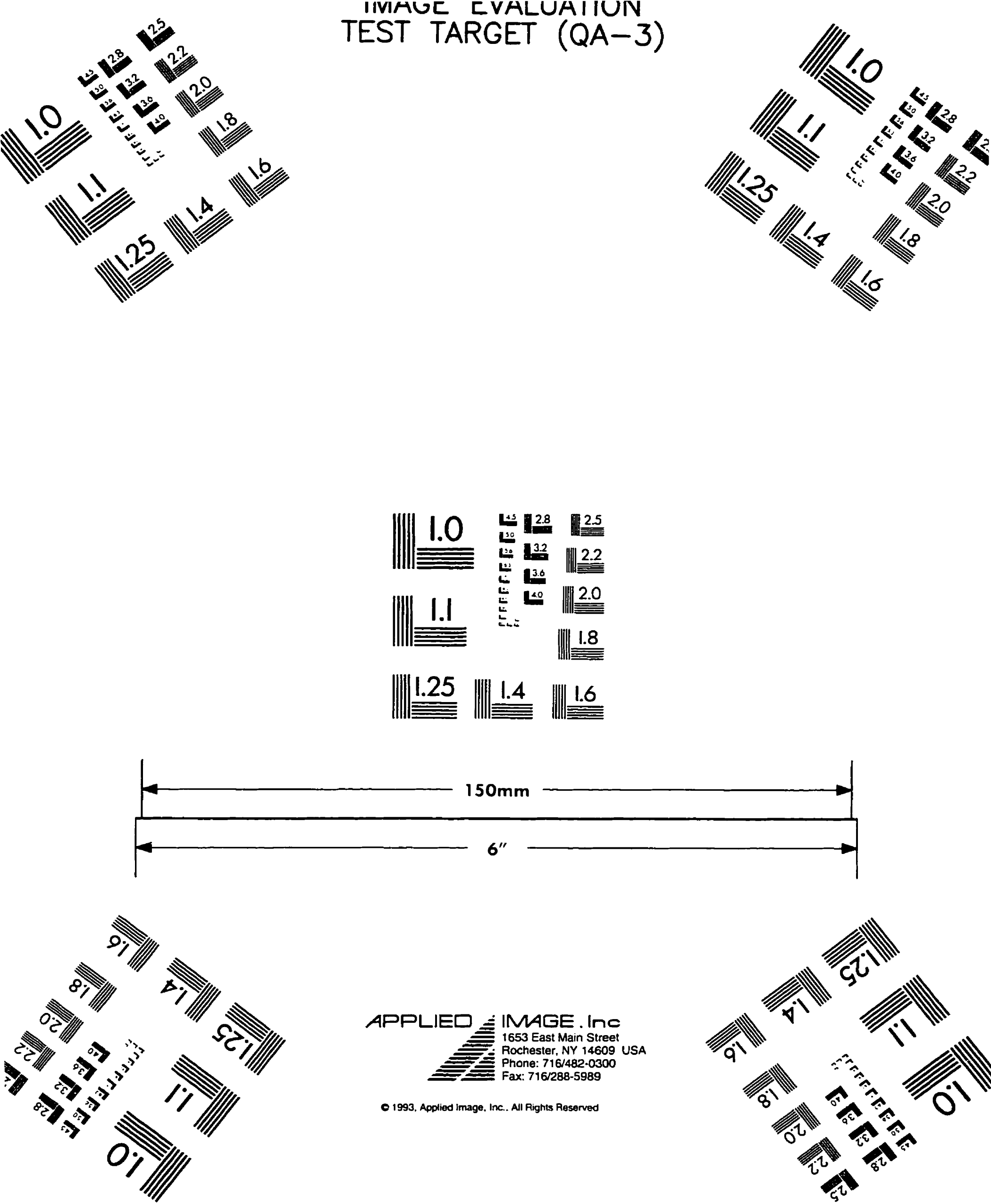

APPLIED $\doteq$ IMAGE. InC

1653 East Main Street

Rochester, NY 14609 USA

Phone: $716 / 482.0300$

Fax: 716/288-5989

C 1993, Applied image, Inc.. All Rights Reserved

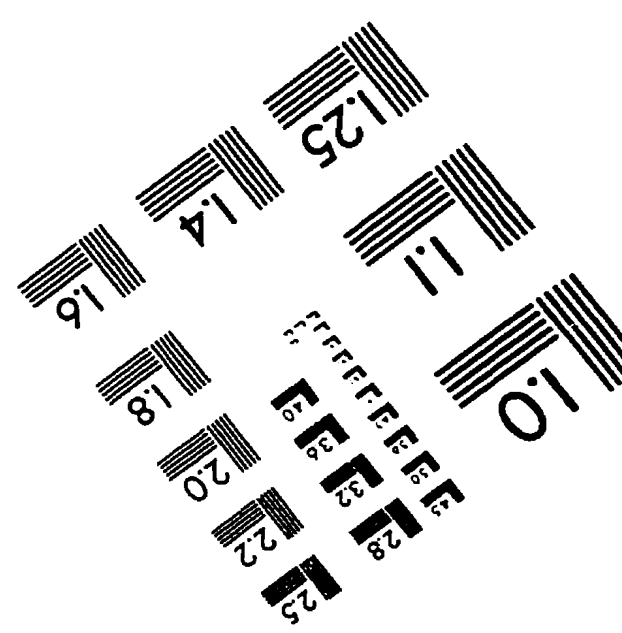

